\title{
Transport Phenomena in Perovskite Vanadate Thin Films
}

\author{
Man Gu \\ Jilin, Jilin, China \\ Bachelor of Science in Materials Science and Engineering, \\ University of Science and Technology of China, 2009
}

\author{
A Dissertation presented to the Graduate Faculty \\ of the University of Virginia in Candidacy for the Degree of \\ Doctor of Philosophy \\ Department of Physics \\ University of Virginia \\ December, 2014
}


Dedicated to my beloved family

For their endless love, support and encouragement 


\section{Table of Contents}

Table of Contents .......................................................................................................................... i

Abstract................................................................................................................................... vi

Acknowledgement ............................................................................................................................. viii

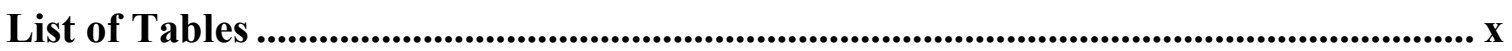

List of Figures........................................................................................................................ xi

Chapter 1 Introduction ...................................................................................................... 1

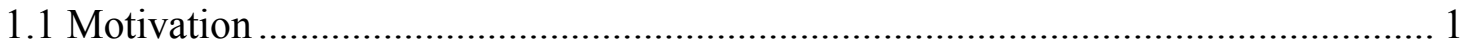

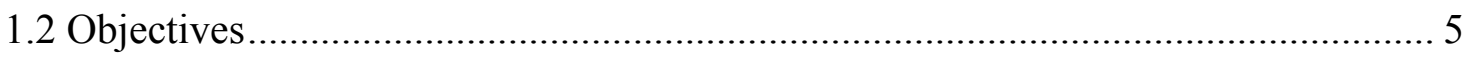

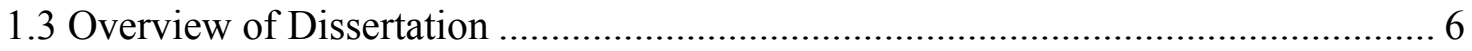

Chapter 2 Background ................................................................................................................ 8

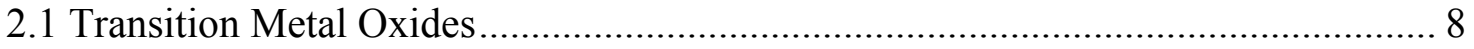

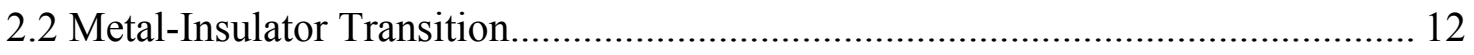

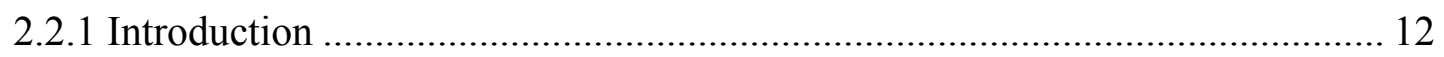

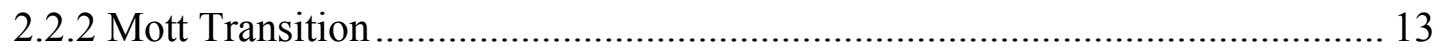

2.2.3 Peierls Transition................................................................................... 19

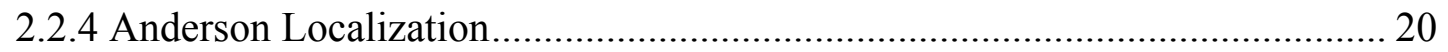

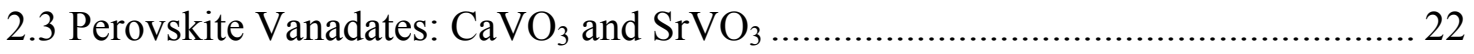




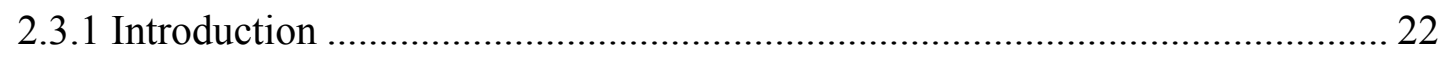

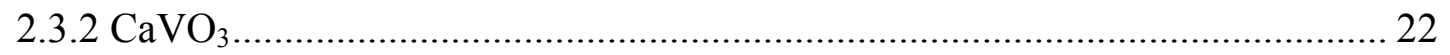

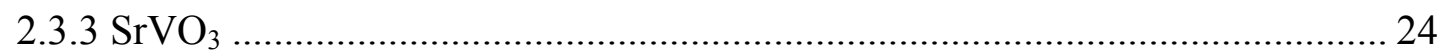

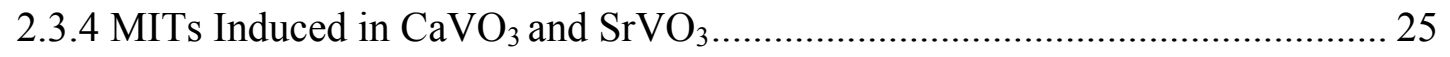

2.4 Thin Film Growth .................................................................................... 27

2.4.1 Pulsed Electron-Beam Deposition Procedures .......................................... 27

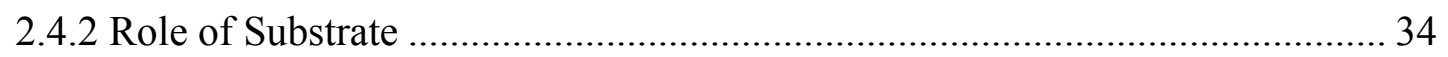

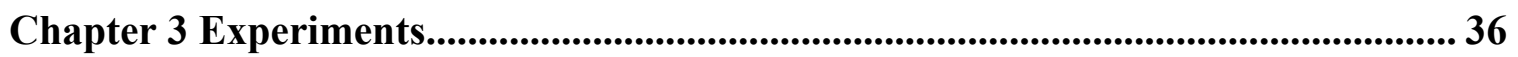

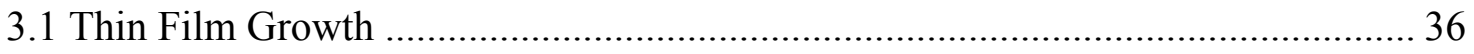

3.1.1 Pulsed Electron-Beam Deposition Setup..................................................... 36

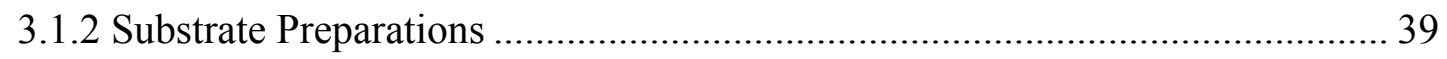

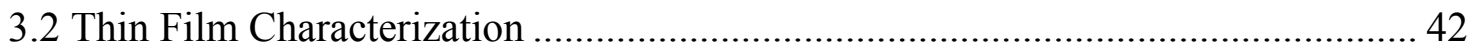

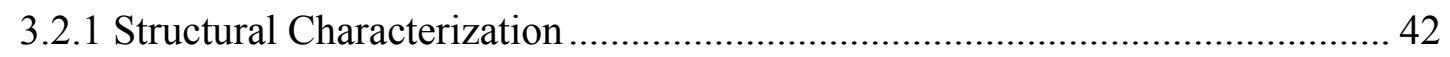

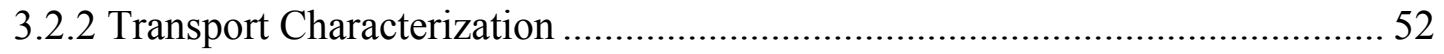

Chapter 4 Epitaxial Vanadate Thin Films ...................................................................... 55

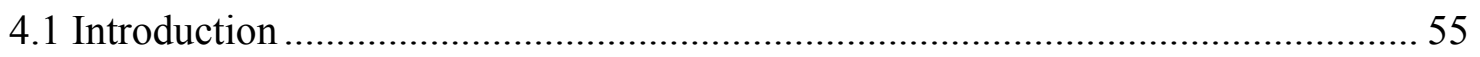

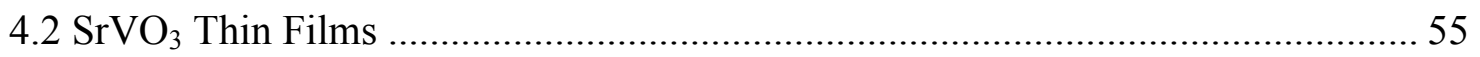

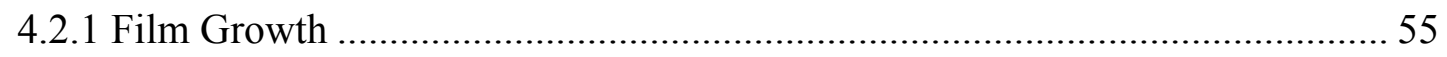

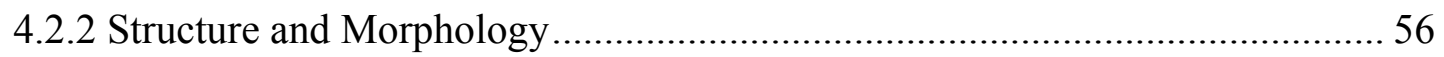




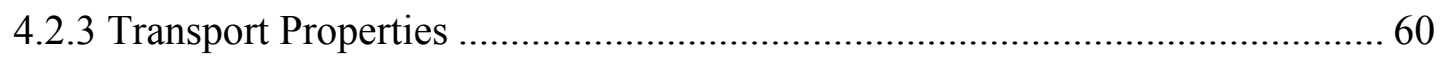

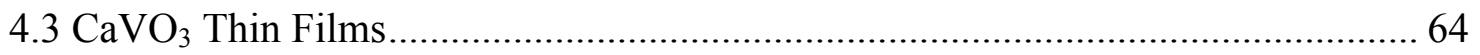

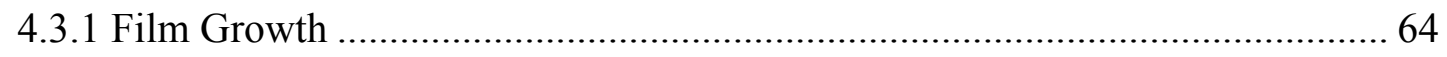

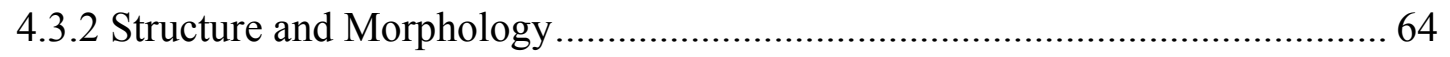

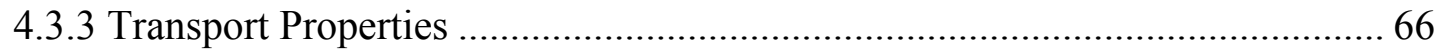

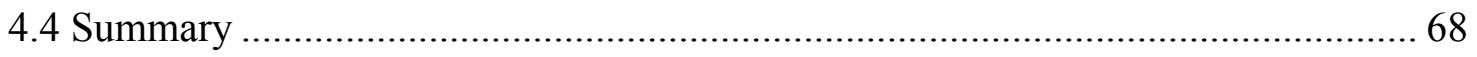

Chapter 5 Two-Dimensional Mott Insulators in Vanadates ........................................ 70

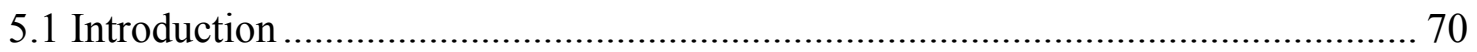

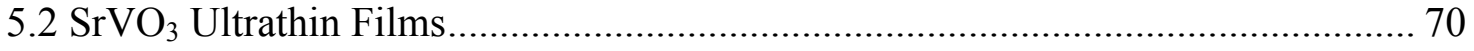

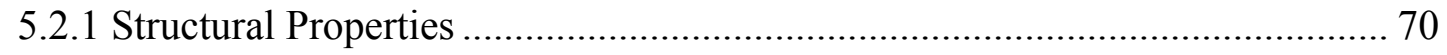

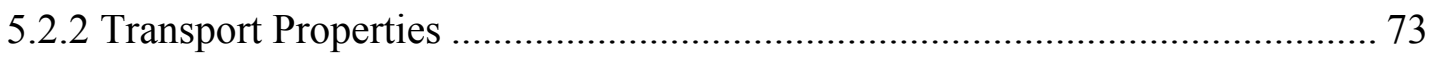

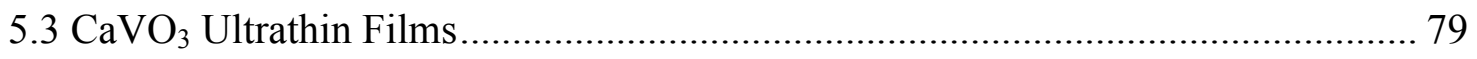

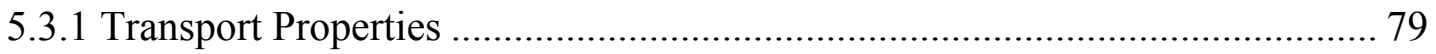

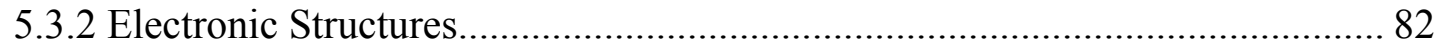

5.4 MIT Mechanism ........................................................................................... 83

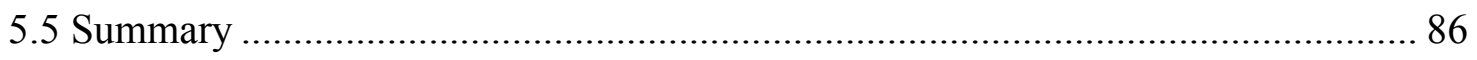

Chapter 6 MIT in $\mathrm{SrTi}_{1-x} \mathrm{~V}_{x} \mathrm{O}_{3}$ Thin Films ........................................................... 88

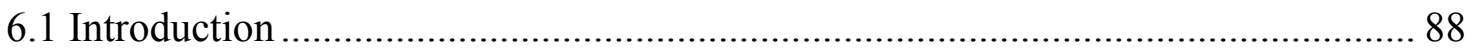

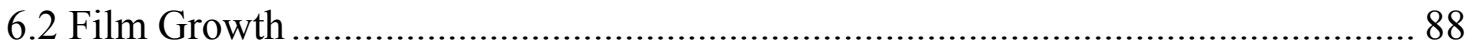




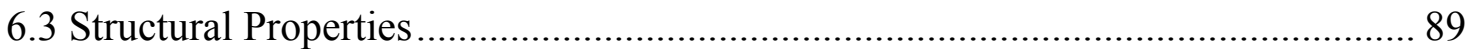

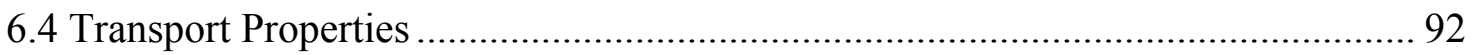

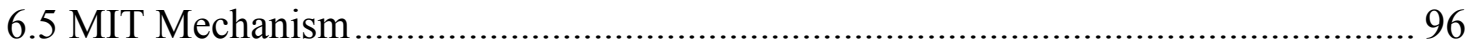

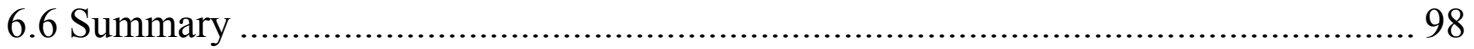

Chapter 7 Transport in $\mathrm{SrVO}_{3} / \mathrm{SrTiO}_{3}$ Superlattices .................................................. 100

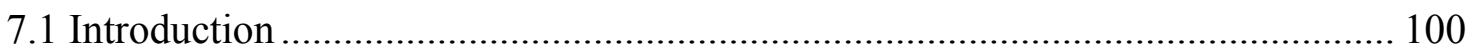

7.2 SVO/STO Superlattice Growth ............................................................ 101

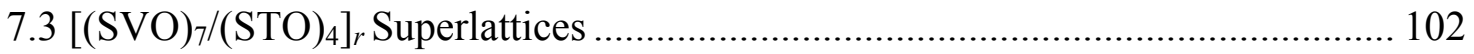

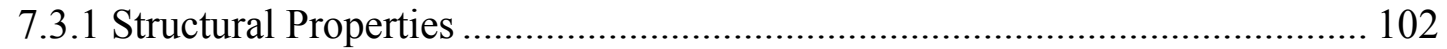

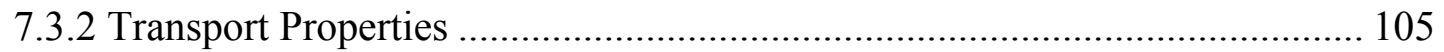

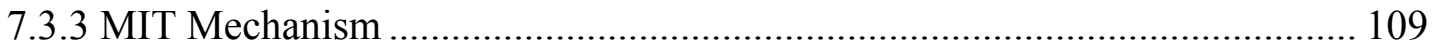

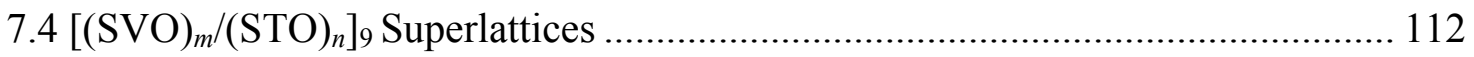

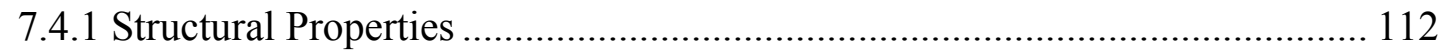

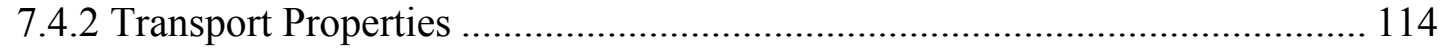

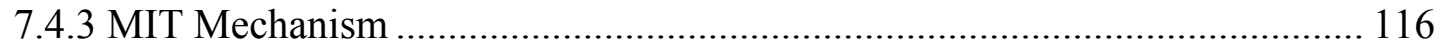

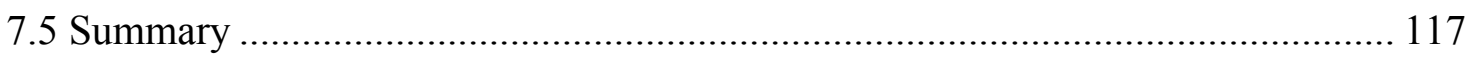

Chapter 8 Summary and Future Work ..................................................................... 119

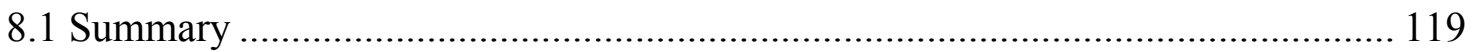

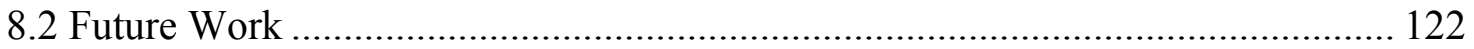




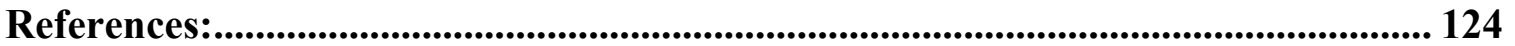




\section{Abstract}

The metal-insulator transition (MIT) in transition metal oxides (TMOs) has been a topic of long-standing interest in condensed matter physics. Recent advances in thin film technology have greatly motivated the scientific community to tailor the MIT for nanoelectronic device applications. This dissertation is focused on exploring the transport phenomena in the perovskite vanadate thin films. $\mathrm{CaVO}_{3}(\mathrm{CVO})$ and $\mathrm{SrVO}_{3}(\mathrm{SVO})$ are chosen because they are typical strongly correlated oxides on the metallic side of a MIT, but their intrinsic physical properties have not yet been studied comprehensively due to the great challenges presented by the synthesis of these materials. In this dissertation, high quality epitaxial vanadate thin films synthesized by a novel pulsed electron-beam deposition (PED) technique enable a careful study on the control of the MIT. Methods to systematically control the MIT in these materials have been demonstrated, including dimensional confinement, chemical doping, and superlattice structures. Techniques such as soft x-ray spectroscopy and magneto-transport measurements are employed to elucidate the underlying mechanisms for the induced MITs.

The transport properties of the vanadate films were studied as a function of the film thickness. A temperature-driven MIT was observed in the SVO films with thicknesses below $6.5 \mathrm{~nm}$, and CVO films below $4 \mathrm{~nm}$, whereas thicker films were metallic with the resistivity following the $T^{2}$ law corresponding to a Fermi liquid system. This work represents the first transport study to show the MIT in the vanadates induced by the dimensional crossover from a three-dimensional metal to a two-dimensional Mott insulator, as the resulting reduction in the effective bandwidth opens a bandgap at the 
Fermi level. The magneto-transport measurements also confirmed the MIT is due to the electron-electron interactions other than disorder-induced localization.

The B-site doping in the SVO films with $\mathrm{Ti}^{4+}$ ions was investigated for the first time to explore the chemical doping effects. The transport study in the full composition range from $\mathrm{SVO}_{\text {to }} \mathrm{SrTiO}_{3}$ (STO) revealed a temperature-driven MIT in the $x=0.67$ film at $95 \mathrm{~K}$. The films with higher vanadium concentration were metallic, whereas the ones with lower vanadium concentration were semiconducting following Mott's variable range hopping mechanism. The mechanisms behind the observed MIT are complicated due to competing effects among electron correlation, disorder, and percolation. Percolation likely plays a major role in the system, for which the transport properties are determined by the topology of the coexisting metallic and insulating regions.

The transport properties of $\mathrm{SrVO}_{3} / \mathrm{SrTiO}_{3} \quad(\mathrm{SVO} / \mathrm{STO})$ superlattices were comprehensively investigated by varying the number of repetitions of the SVO/STO bilayers and the thickness of individual SVO and STO layers. The SVO layer embedded in the superlattices showed a great enhancement in the conductivity, which is a further indication of electronic phase separation in the vanadate ultrathin layers. The transport behaviors in the superlattices can also be described as percolation phenomena. The coupling between SVO layers creates more conduction pathways with increasing number of repetitions, resulting in a crossover from insulating to metallic behavior. Also, an increase in the SVO layer thickness along with a reduction in the STO layer thickness may cause an increase in the interlayer electron hopping, leading to the crossover from insulating to a metallic behavior. 


\section{Acknowledgement}

This dissertation would not have been possible without the help of many people, to whom I would like to express my heartfelt thanks. First and foremost, my deepest gratitude goes to my advisors Prof. Stuart Wolf and Prof. Jiwei Lu, for their support and guidance throughout my graduate study at University of Virginia. Their excellent mentorship and insightful feedback on my work were invaluable to me. Through the years they have provided me with enthusiasm for science, fruitful discussions on my research projects, a helping hand in the lab when needed, insightful commentary on my writing and presentations, and great opportunities to present my research at conferences. I deeply appreciate their unconditional walk-in hours and sharing their knowledge and experience with me. Their patience and encouragement helped me overcome many difficulties. I am also grateful to my committee Prof. Bellave Shivaram and Prof. Utpal Chatterjee, for their sincere interest in my research and constructive comments. I acknowledge our collaborator Jude Laverock at Boston University for the soft x-ray spectroscopy measurements and helpful discussions.

I would like to thank all the current and former members of the Wolf/Lu research group for their collaboration, assistance, and friendship, including Yonghang Pei, Salinporn Kittiwatanakul, Nattawut Anuniwat, Linqiang Luo, Yuhan Wang, Keren Freedy, Nam Dao, Ryan Comes, Hongxue Liu, Yishen Cui, Wenjing Yin, Wenbin Fan, Wei Chen, David Kirkwood, and Melissa Commisso Dolph. They have proven to be excellent colleagues and great friends, I appreciate the time I spent with them. I would also like to acknowledge all of the members of the faculty and staff of Department of 
Physics and Department of Materials Science and Engineering, particularly, Tonya Reynolds, Jeffrey Fox, Tommy Eanes, Sherri Sullivan, Tammie Shifflett, Dawn Shifflett, Helen McLaughlin, Beth Guyton, Vicky Ingram, Chris Floyd, David Wimer, and Larry Suddarth for all the support throughout my graduate study.

I would like to thank my fellow graduate students for their invaluable support and suggestions throughout this journey. I am also very grateful to my dearest friends who never hesitated to devote their valuable time to me. Graduate school would not have been the same without my loving friends at my side. I dare not risk missing to mention anyone's name, thank you all for being there for me, and I will always cherish all the beautiful memories we have shared in the past five years at University of Virginia.

Last but not least, my thanks go to my beloved family, especially my mother, Yanlai Gu, who has supported and encouraged me not to give up on my dreams. Without my mother's unconditional love, I would not be where I am today. 


\section{List of Tables}

Table 2-1 Specification of Neocera PEBS-20. (Adapted from Ref. 80) …...................... 29

Table 4-1 Substrate lattice parameters and mismatch to SVO....................................... 56

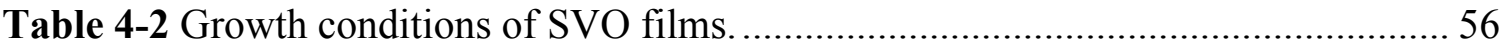

Table 4-3 Out-of-plane and in-plane epitaxial strain in the $25 \mathrm{~nm}$ SVO films grown on

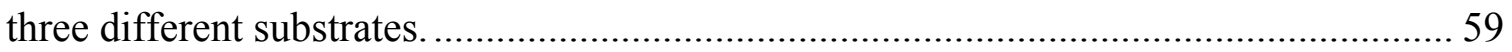

Table 4-4 Transport data of $25 \mathrm{~nm}$ SVO films grown on the three different substrates. . 62

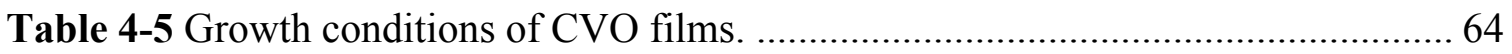

Table 5-1 Out-of-plane lattice parameters of SVO films with various thicknesses......... 73

Table 5-2 Transport properties of SVO films with various thicknesses deposited on the

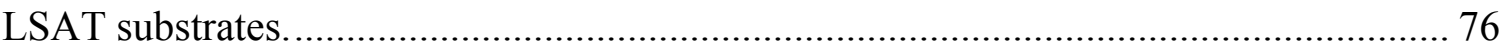

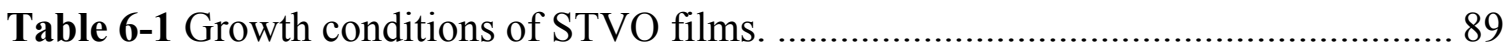

Table 6-2 Lattice parameters of the STVO $(0 \leqslant x \leqslant 1)$ thin films. (For all the films, in-

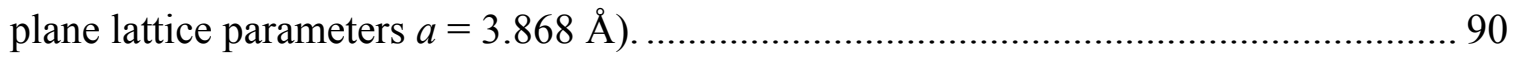

Table 6-3 Transport properties of the STVO $(0.33 \leqslant x \leqslant 1)$ thin films. ....................... 94

Table 7-1 Growth conditions of SVO/STO superlattices............................................... 101

Table 7-2 Transport data of the $\left[(\mathrm{SVO})_{7} /(\mathrm{STO})_{4}\right]_{r}(1 \leqslant r \leqslant 9)$ superlattices............... 106

Table 7-3 Transport behaviors in the $\left[(\mathrm{SVO})_{7} /(\mathrm{STO})_{4}\right]_{r}(1 \leqslant r \leqslant 9)$ superlattices in

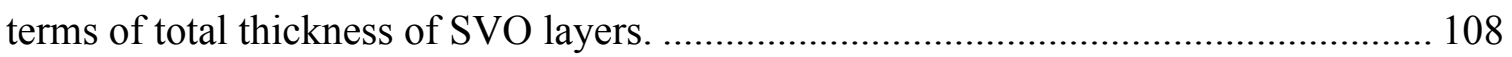

Table 7-4 Transport data of the $\left[(\mathrm{SVO})_{m} /(\mathrm{STO})_{n}\right]_{9}$ superlattices.................................. 116 


\section{List of Figures}

Figure 1-1 (a) Transition temperature $T_{M I T}$ of some selected oxides in bulk. (b) Demonstration of a switching device utilizing MIT in TMOs. (Adapted from Ref. 9)...... 4

Figure 2-1 The rich functional properties in $\mathrm{ABO}_{3}$ perovskites arising from the complex interplay between spin, charge, orbital and lattice degrees of freedom. The ideal

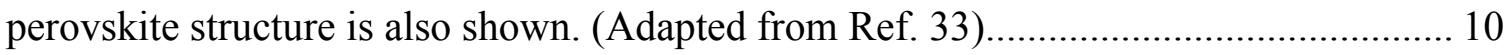

Figure 2-2 Electronic band structures of a metal and an insulator. Fermi level $E_{F}$ is also shown. 13

Figure 2-3 Metal-insulator transition driven by strong electron correlation effects. 14

Figure 2-4 Schematic energy levels for (a) Mott-Hubbard insulator and (b) charge transfer insulator. (Adapted from Ref. 4) (c) Schematic phase diagram of $\mathrm{ABO}_{3}$ perovskites for $\mathrm{A}=\mathrm{Y}^{3+}, \mathrm{La}^{3+}, \mathrm{Ca}^{2+}$ and $\mathrm{Sr}^{2+}$ as a function of $\mathrm{U} / \mathrm{W}, \Delta / \mathrm{W}$ and electron band filling. The thin lines connect A-site cations to B-site cations, and the thick lines represent the solid solutions. (Adapted from Ref. 30) ................................................. 16

Figure 2-5 (a) Bandwidth-controlled MIT (BC-MIT) and filling-controlled MIT (FCMIT) in a Mott-Hubbard system. (Reproduced from Ref. 4) (b) Metal-insulator phase diagram of some perovskite titanates and vanadates as a function of $U / W$ and electron band filling. (Adapted from Ref. 40) 18

Figure 2-6 (a) One-dimensional metal with lattice constant $a$ and (b) Peierls insulator with the periodic modulation (charge-density-wave) $\lambda=\pi / \mathrm{k}_{\mathrm{F}}$ and their band structures (c) and (d). (Adapted from Ref. 42). 19 
Figure 2-7 Schematic density of states in a disordered system, showing mobility edges separating localized and extended states. (Adapted from Ref. 42 ) 21

Figure 2-8 Orthorhombically distorted $\mathrm{GdFeO}_{3}$-type structure, $\theta$ is the $\mathrm{B}-\mathrm{O}-\mathrm{B}$ bond angle. (Adapted from Ref. 51). 22

Figure 2-9 Electrical resistivity vs. temperature of (a) single-crystalline CVO with increasing oxygen off-stoichiometry. (Adapted from Ref. 52) (b) 45nm CVO films grown on $\mathrm{SrTiO}_{3}(\mathrm{STO}),\left(\mathrm{LaAlO}_{3}\right)_{0.3}\left(\mathrm{Sr}_{2} \mathrm{AlTaO}_{6}\right)_{0.7}(\mathrm{LSAT})$ and $\mathrm{LaAlO}_{3}(\mathrm{LAO})$ substrates. A $\rho$

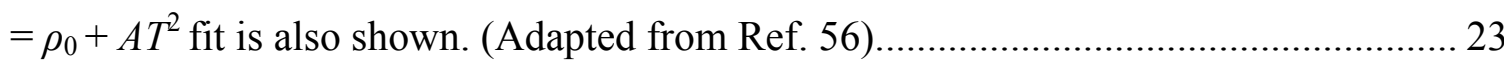

Figure 2-10 Electrical resistivity vs. temperature of $10 \mathrm{~nm}$ SVO films grown on $\mathrm{AlO}_{2}$ terminated $\mathrm{LaAlO}_{3}\left(\mathrm{AlO}_{2}-\mathrm{LAO}\right), \mathrm{SrTiO}_{3}(\mathrm{STO}), \mathrm{LaO}$-terminated $\mathrm{LaAlO}_{3}(\mathrm{LaO}-\mathrm{LAO})$

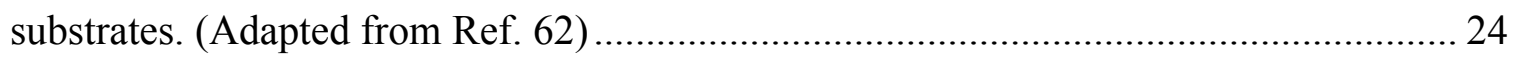

Figure 2-11 (a) In situ valence band spectra of SVO films with thicknesses from 1 to 100 ML. (b) PES spectra of the same SVO films near $E_{F}$. The solid and opened triangles indicate the energy positions of the coherent and incoherent parts, respectively. (Adapted from Ref. 70). 26

Figure 2-12 General Scheme of PED process. (Adapted from Ref. 78) ........................ 28

Figure 2-13 Schematic illustration of various growth modes in thin film growth. (Adapted from Ref. 74). 33

Figure 2-14 Schematic view of epitaxial strain: (a) $a_{f}=a_{s}$, no strain, (b) $a_{f}>a_{s}$, inplane compressive strain, (c) $\mathrm{a}_{\mathrm{f}}<\mathrm{a}_{\mathrm{s}}$, in-plane tensile strain. 35

Figure 3-1 Photograph of the PED system (Neocera, Inc.). 36 
Figure 3-2 View inside the PED chamber showing (a) the relative positions of the electron guns, targets, the sample stage and heater, (b) the plasma plume produced at the surface of $\mathrm{Sr}_{2} \mathrm{~V}_{2} \mathrm{O}_{7}$ target during the deposition. 37

Figure 3-3 Calibration data showing the sample plate temperature as a function of the set temperature. (Adapted from Refs. 90 and 91) 39

Figure 3-4 (a) AFM topography of a representative $\mathrm{SrTiO}_{3}$ (STO) substrate after the treatment. (b) Line profile along the line indicated in (a). 41

Figure 3-5 (a) AFM topography of a representative $\mathrm{LaAlO}_{3}(\mathrm{LAO})$ substrate after the treatment. (b) Line profile along the line indicated in (a).

Figure 3-6 Schematic of the angles $(2 \theta, \omega, \varphi$ and $\psi)$ with respect to the sample plane for a 4-circle goniometer.

Figure 3-7 Schematic of the formulation of Bragg's law in (a) real space and (b) reciprocal space. (Adapted from Refs. 94 and 95.).....

Figure 3-8 Geometry of reciprocal space mapping...... 47

Figure 3-9 A representative XRR profile of a $\mathrm{SrVO}_{3}$ thin film grown on LSAT substrate (blue color), along with a fitted curve (red color).

Figure 3-10 Schematic of AFM instrument. (Adapted from Ref. 102) 51

Figure 3-11 (a) Schematic of a van der Pauw configuration used in the determination of sheet resistance. (b) A representative photo of samples bonded to a PPMS sample puck for resistivity measurements. 53

Figure 3-12 Schematic of (a) Hall effect and (b) sample configuration in the Hall effect measurement. 54 
Figure 4-1 AFM topography images and (inset) line scans of $6.5 \mathrm{~nm} \mathrm{SVO} \mathrm{films} \mathrm{grown}$ on (a) LAO, (b) LSAT and (c) STO substrates. 57

Figure 4-2 (a) Wide range XRD scans of $25 \mathrm{~nm}$ SVO films grown on three different substrates showing that no secondary phases are present. (b) XRD scans around (002) reflection showing Kiessig fringes. The triangles indicate the SVO (002) peak positions.

Figure 4-3 RSM on (103) reflection of $25 \mathrm{~nm} \mathrm{SVO} \mathrm{films} \mathrm{grown} \mathrm{on} \mathrm{(a)} \mathrm{LAO,} \mathrm{(b)} \mathrm{LSAT}$ and (c) STO substrates. 60

Figure 4-4 (a) Sheet resistance vs. temperature for $25 \mathrm{~nm}$ SVO films grown on three different substrates. Temperature dependence of electrical resistivity of the same SVO films grown on (b) LAO, (c) LSAT and (d) STO substrates. $\rho=\rho_{0}+A T^{2}$ fits are also shown. 61

Figure 4-5 Temperature dependence of carrier density and mobility from Hall effect measurements of the same $25 \mathrm{~nm}$ SVO films grown on (a) LAO, (b) LSAT, and (c) STO substrates.

Figure 4-6 (a) AFM topography image and (b) line scan of a $5.2 \mathrm{~nm}$ CVO film grown on STO substrate (scan area: $\left.1.0 \times 1.0 \mu \mathrm{m}^{2}\right)$. 65

Figure 4-7 XRD scan around (002) reflection of a CVO film with thickness of $53 \mathrm{~nm}$. (Inset) Wide range scan showing that no secondary phases are present. 66 Figure 4-8 (a) Electrical resistivity vs. temperature for CVO film with thickness of 62 nm. A $\rho=\rho_{0}+A T^{2}$ fit is also shown. (b) Temperature dependence of carrier mobility and carrier density of the same $62 \mathrm{~nm}$ CVO film.... 68 
Figure 5-1 (a) XRD scans around (002) reflection of SVO films with various thicknesses, the SVO film peaks are indicated by the arrows. RSM on (103) reflection of (b) $25 \mathrm{~nm}$ and (c) $15.3 \mathrm{~nm}$ SVO films. 72

Figure 5-2 (a) Electrical resistivity vs. temperature for SVO films with thickness ranging from $3 \mathrm{~nm}$ to $25 \mathrm{~nm}$. Arrows indicate the local minima of resistivity. (Inset) $T_{M I T}$ vs. film thickness with a linear fit to the data. Temperature dependence of electrical resistivity on a different scale for SVO films with thicknesses of (b) $6.5 \mathrm{~nm}$, (c) $5.7 \mathrm{~nm}$ and (d) $3 \mathrm{~nm}$. (Inset) Logarithm of conductivity below $30 \mathrm{~K}$ as a function of $T^{1 / 4}$ for the same (b) 6.5 $\mathrm{nm}$, (c) $5.7 \mathrm{~nm}$ and (d) $3 \mathrm{~nm}$ SVO films. A linear fit to the data is also shown. 75 Figure 5-3 (a) Normalized out-of-plane MR of the $5.7 \mathrm{~nm}$ SVO ultrathin film between 5 and $50 \mathrm{~K}$ with the field up to $7 \mathrm{~T}$. The MR is proportional to $B^{2}$ and the parabolic fits at each temperature are also shown in solid lines. (b) Temperature dependence of carrier density and mobility from Hall measurements of the same $5.7 \mathrm{~nm}$ SVO ultrathin film. . 78 Figure 5-4 Sheet resistance vs. temperature for CVO films with thickness of 2, 4, 8.5, 62 $\mathrm{nm}$, STO film with thickness of $18 \mathrm{~nm}$, and STO substrate annealed at the temperature of deposition in the same environment. 80

Figure 5-5 I-V measurements of $2 \mathrm{~nm}$ CVO film in the temperature range of 2-100 K. (Inset) Optical picture of the electrical contacts configuration. 81

Figure 5-6 XAS spectra measured at (a) V $L$-edge and (b) Ca $L$-edge for CVO single crystal (SC) reference and STO capped CVO films with thickness of $4 \mathrm{~nm}$ and $46 \mathrm{~nm} . .83$ Figure 5-7 Schematic of the bandwidth-controlled MIT (BC-MIT). (Reproduced from Ref. 119) 85 
Figure 6-1 (a) XRD scans for the STVO $(0 \leq x \leq 1)$ thin films grown on LSAT $(001)$ substrates. (b) Calculated unit cell volume as a function of $x$. The straight line represents the Vegard's law. (c)-(i) The reciprocal space mapping on (103) reflection for the same STVO thin films 91

Figure 6-2 (a) Electrical resistivity vs. temperature for the STVO $(0.33 \leq x \leq 1)$ thin films. (b) Carrier density from room-temperature Hall measurements for the STVO $(0.5 \leq$ $x \leq 1$ ) thin films. 92

Figure 6-3 Temperature dependence of electrical resistivity for the STVO thin films, (a) $x=1$ and 0.83 , (b) $x=0.67, \rho=\rho_{0}+A T^{2}$ fits are also shown. Logarithm of conductivity as a function of $T^{1 / 4}$ with a linear fit to the data, (c) $x=0.5,2 \mathrm{~K} \leq T \leq 300 \mathrm{~K}$, (d) $x=0.33$, $110 \mathrm{~K} \leq T \leq 300 \mathrm{~K}$

Figure 6-4 Normalized out-of-plane MR of the STVO $(x=0.67)$ thin film between $2 \mathrm{~K}$ and $30 \mathrm{~K}$ with the field up to $7 \mathrm{~T}$. The parabolic fits at each temperature are also shown in lines. 96

Figure 7-1 (a) Wide range out-of-plane XRD scans for the $\left[(\mathrm{SVO})_{7} /(\mathrm{STO})_{4}\right]_{r}$ superlattices with $r=1,2,3,5,7,9$ grown on (001)-oriented LSAT substrates showing that no secondary phases are present. The first-order satellite peaks are noted. (b) XRD scan around the (002) reflection of SVO/STO superlattice with $r=9$ showing Kiessig fringes, and satellite peaks up to second order are noted. (Inset) Rocking curves around the (002) zeroth-order superlattice peak and the underlying LSAT substrate peak. (c) The reciprocal space mapping on (103) reflection for the same SVO/STO superlattice with $r$ $=9$. 104 
Figure 7-2 (a) Sheet resistance as a function of temperature for the $\left[(\mathrm{SVO})_{7} /(\mathrm{STO})_{4}\right]_{r}$ superlattices with $r=1,2,3,5,7,9$. Temperature dependence of electrical resistivity for the same set of SVO/STO superlattices with (b) $r=9$, (c) $r=2, \rho=\rho_{0}+A T^{2}$ fits are also shown. (d) Logarithm of conductivity as a function of $T^{1 / 4}$ with a linear fit to the data for the SVO/STO bilayer $(r=1)$.

Figure 7-3 Normalized out-of-plane MR of the $\left[(\mathrm{SVO})_{7} /(\mathrm{STO})_{4}\right]_{r}$ superlattices with (a) $r$ $=9$ and (b) $r=2$ between $5 \mathrm{~K}$ and $20 \mathrm{~K}$ with the field up to $7 \mathrm{~T}$. The parabolic fits at each temperature are also shown in lines.

Figure 7-4 (a) The inverse of the sheet resistance of the SVO/STO superlattices as a function of the number of repetitions measured at three different temperatures. The linear fits to the data are also shown. (b) Calculated sheet resistance of the individual SVO layer embedded in the superlattices with $2 \leq r \leq 9$ and measured sheet resistance of the $\mathrm{SVO} / \mathrm{STO}$ bilayer $(r=1)$.

Figure 7-5 (a) Wide range out-of-plane XRD scans for the $\left[(\mathrm{SVO})_{m} /(\mathrm{STO})_{n}\right]_{9}$ superlattices grown on (001)-oriented LSAT substrates showing that no secondary phases are present and the first-order satellite peaks are noted. (b) Calculated average out-ofplane lattice parameter of the superlattices as a function of $m /(m+n)$. A linear fit is also shown.

Figure 7-6 (a) Sheet resistance as a function of temperature for the $\left[(\mathrm{SVO})_{m} /(\mathrm{STO})_{n}\right]_{9}$ superlattices. Temperature dependence of electrical resistivity for the same set of SVO/STO superlattices with (b) $m=13, n=4, \rho=\rho_{0}+A T^{2}$ fit is also shown, and (c) $m=$ 
6, $n=5$. (d) Logarithm of conductivity as a function of $T^{1 / 2}$ for the superlattice with $m=$ $2, n=7$. A linear fit to the data is also shown. ............................................................ 115 


\section{Chapter 1 Introduction}

\subsection{Motivation}

Silicon-based devices have been the mainstay of the electronics industry. According to Moore's law, the number of transistors on an integrated circuit doubles approximately every two years. This exponential growth continues today as chip geometries become smaller and smaller, approaching fundamental physical limits. ${ }^{1}$ To sustain an increase in the degree of integration and to further improve the device performance, novel electronic materials beyond the scaling limits of silicon technology are urgently needed.

Transition metal oxides (TMOs) offer a rich variety of intriguing physical properties that are not obtainable in conventional semiconductors. In recent years, major improvements in oxide thin film synthesis techniques have provided the opportunities to tailor the unprecedented wealth of functionalities for a wide range of potential nanoscale device applications. Many striking properties in thin-film TMOs have shown great promise for next-generation devices including switches, sensors, memories and transistors, opening up a new field of oxide nanoelectronics. ${ }^{2,3}$

The metal-insulator transition (MIT) in TMOs is one of the most fascinating phenomena in condensed matter physics. Experimental and theoretical efforts to understand the MIT have been ongoing for decades. ${ }^{4}$ Underlying physical mechanisms

include electron-electron interactions (Mott transition ${ }^{5,6}$ ), electron-phonon interactions 
$\left(\right.$ Peierls transition $\left.{ }^{7}\right)$ and disorder-induced localization (Anderson localization ${ }^{8}$ ). The ultrafast nature of the phase transition and the resulting changes in the material's physical properties have generated a considerable interest in utilizing MIT as a new class of switching devices. ${ }^{9}$

Fig. 1-1 (a) shows some TMOs exhibiting temperature-driven MITs. The transition temperatures $T_{M I T}$ of different materials vary over a wide temperature range. Among these materials, $\mathrm{VO}_{2}$ has the $T_{M I T}$ closest to room temperature, which is $\sim 340 \mathrm{~K}$ in bulk, and shows several orders of magnitude change in the resistivity across the transition, making it a compelling candidate for novel switching devices operable near room temperature. ${ }^{10}$ Other than thermal triggering, MIT can also be triggered by electrical $^{11,12}$, optical ${ }^{13,14}$, magnetic ${ }^{11}$ and strain $^{15}$ excitations. As demonstrated in Fig. 1-1 (b), the central concept of devices utilizing MIT is a switch with the high-resistance, insulating OFF state and low-resistance, metallic ON state on both sides of the MIT. The switching can thus be controlled by an external perturbation that triggers the MIT.

Numerous efforts have been made to realize device concepts utilizing MIT, including ultrafast electrical switches ${ }^{16}$, memristive devices ${ }^{17}$, optical modulators ${ }^{18}$ and thermal sensors ${ }^{19}$. These novel oxide-based devices have demonstrated superior characteristics to conventional semiconductors, providing great opportunities for future electronics. Recently, special attention has been focused on utilizing the Mott transition for a novel Mott field effect transistor (MottFET). ${ }^{20}$ It has been demonstrated that a Mott material, such as $\mathrm{VO}_{2}$, can be employed as the channel in a MottFET with an applied external gate voltage switching the channel resistance between the insulating OFF state 
and the metallic ON state. ${ }^{21,22,23}$ Such a MottFET could offer significant advantages over the conventional metal-oxide-semiconductor field effect transistor (MOSFET), including large on/off ratio, steep subthreshold switching, and femtosecond switching speed. ${ }^{24}$ It is predicted that the channel length of a MottFET can be scaled down to less than $10 \mathrm{~nm}$ owing to the short screening length of the Mott materials. ${ }^{25}$ The realization of the MottFET could significantly impact the future of electronics, as the conventional MOSFET is approaching its intrinsic physical limits. Therefore, establishing new approaches to predict, understand, and control the MIT is crucial for both fundamental physics and technological applications. 
(a)
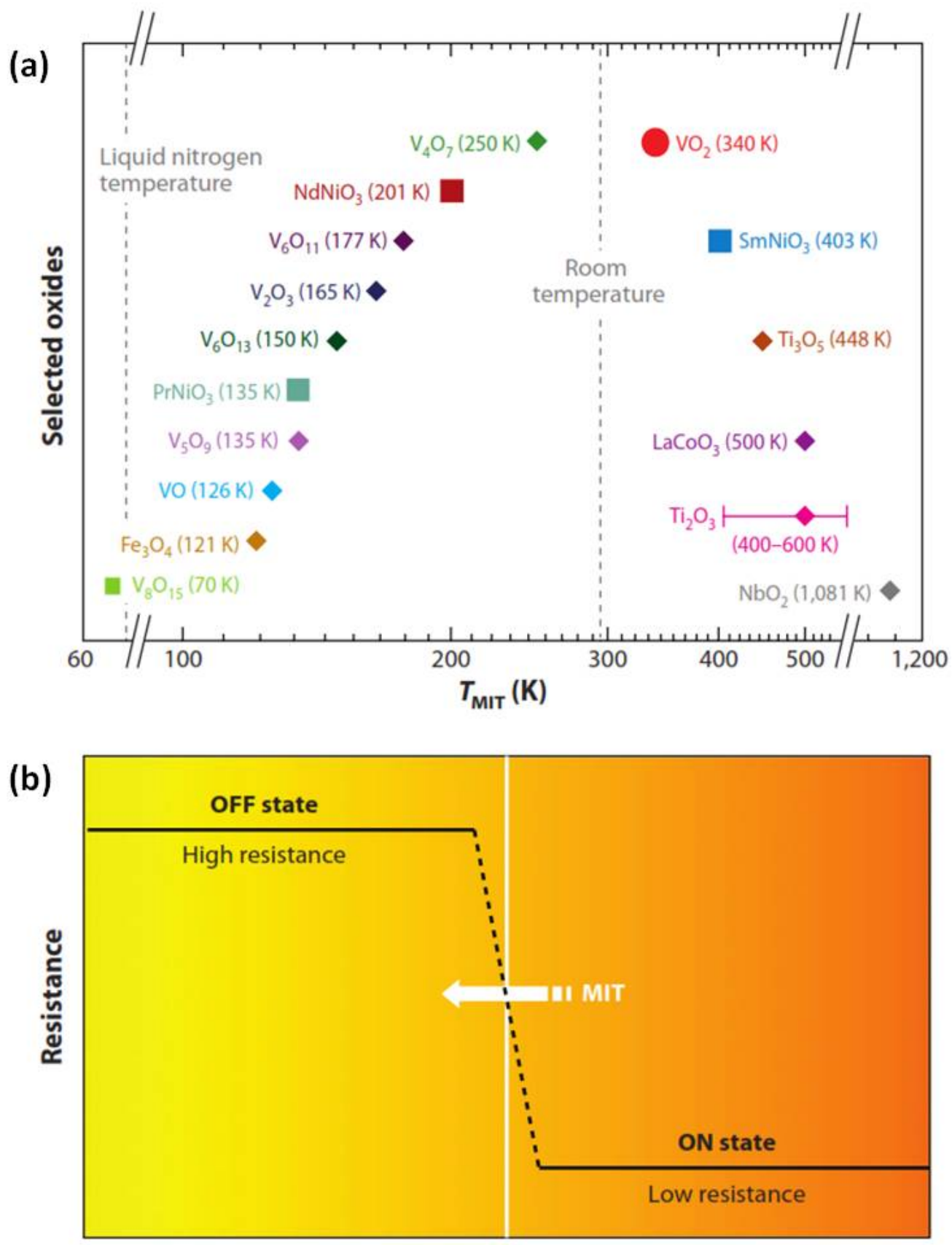

Thermal, electrical, optical, magnetic, or strain-driven excitations

Figure 1-1 (a) Transition temperature $T_{M I T}$ of some selected oxides in bulk. (b) Demonstration of a switching device utilizing MIT in TMOs. (Adapted from Ref. 9) 


\subsection{Objectives}

To explore the MIT in nanoscale TMOs and assess the potential for future device applications, it is essential to understand and control the transport properties of TMO thin films. The perovskite vanadates $\mathrm{CaVO}_{3}(\mathrm{CVO})$ and $\mathrm{SrVO}_{3}(\mathrm{SVO})$ are typical strongly correlated electron systems on the metallic side of a MIT. ${ }^{26}$ However, due to the great challenges in the synthesis of these materials, the intrinsic physical properties of CVO and SVO thin films have not yet been studied comprehensively. In this work, we employ a novel pulsed electron-beam deposition (PED) tool to optimize epitaxial growth of the perovskite vanadate thin films for atomic-level control over film thicknesses, structural perfection, cation and oxygen stoichiometry, and low defect densities.

This dissertation is focused on exploring the transport phenomena in the perovskite vanadate thin films. Both $\mathrm{CVO}$ and SVO are Mott materials where the electron-electron interactions play an important role on their transport properties, therefore, the MIT in these materials can be controlled by modifying the bandwidth or the band filling. ${ }^{4}$ This dissertation aims to engineer these Mott materials to be as close as possible to the MIT. The successful growth of high quality epitaxial thin films provides new methods to control the MIT in ways not possible with bulk materials. In this work, I demonstrate the systematic control of the MIT in the CVO and SVO thin films and determine the role of lattice strain, dimensional confinement and chemical doping in the transport properties of these materials. This study will lead to further understanding of the fundamental physics behind the MIT in the perovskite vanadate thin films, 
simultaneously providing new perspectives on future device applications of the Mott materials.

\subsection{Overview of Dissertation}

The work presented in this dissertation is an experimental study exploring the transport behaviors of the perovskite vanadate thin films and control over the MIT in these materials, addressing the underlying mechanisms.

Chapter 2 provides a brief overview of the rich phenomena in TMOs, an introduction to the MIT and the potential device applications, and the background information on the materials of interest, including CVO and SVO.

Chapter 3 describes the experimental setup and procedure for the growth of epitaxial oxide thin films using the pulsed electron-beam deposition tool, as well as the structural and transport characterization techniques.

Chapter 4 presents the experimental results for the epitaxial high quality CVO and SVO thin films grown by PED, the film structure, morphology and transport properties are discussed.

Chapter 5 describes two-dimensional Mott insulators in CVO and SVO ultrathin films with thicknesses of only a few nanometers. The temperature dependence of the film transport properties is studied as a function of the film thickness and a dimensionalcrossover-driven MIT is demonstrated. Furthermore, the origin of the insulating state in the ultrathin films is addressed. 
Chapter 6 presents a systematic study of the transport properties of the epitaxial $\mathrm{SrTi}_{1-x} \mathrm{~V}_{x} \mathrm{O}_{3}$ thin films over the whole composition range of $0 \leq x \leq 1$. A temperaturedriven MIT occurs in the $x=0.67$ film at $95 \mathrm{~K}$. The films with higher vanadium concentration are metallic, and the ones with lower vanadium concentration are semiconducting. The possible mechanisms behind the emergent MIT are discussed.

Chapter 7 demonstrates two different approaches for manipulating the transport behaviors of $\mathrm{SrVO}_{3} / \mathrm{SrTiO}_{3}(\mathrm{SVO} / \mathrm{STO})$ superlattice structures by varying the number of repetitions of the SVO/STO bilayers and the thickness of individual SVO and STO layers. The underlying physical mechanisms for the transport behaviors of the different structures are proposed.

Chapter 8 summarizes the key findings and discusses the possible directions for future work. 


\section{Chapter 2 Background}

\subsection{Transition Metal Oxides}

Transition metal oxides (TMOs) represent one of the most fascinating classes of functional materials: The complex interplay between spin, charge, orbital and lattice degrees of freedom generate a rich spectrum of intriguing electrical and magnetic properties, including colossal magnetoresistance, metal-insulator transitions and hightemperature superconductivity. The extreme sensitivity of these properties to structural distortions and crystal chemistry offers great opportunities for tailoring and engineering new functionalities as alternatives to a wide range of existing technological applications.

The incredible diversity of physical phenomena in TMOs is due to the $d$ electrons. In these oxide compounds, the transition metal ion forms covalent bonds with oxygen, leaving only $d$ electrons in its outer shell. Therefore, the basic electronic structure has the partially filled $d$ bands as frontier bands. The spatially constrained $d$ orbitals form narrow $d$ bands with high effective masses and strong on-site Coulomb interactions. These $d$ electrons are highly correlated and cannot be described as embedded in a static mean field generated by the other electrons. The study of the physical phenomena arising from strong electron correlation in TMOs is one of the most attractive fields in condensed matter physics. ${ }^{4}$

One family of TMOs that has received considerable attention is the $\mathrm{ABO}_{3}$ compounds that crystallize into the perovskite structure where $\mathrm{A}$ is an alkaline or rare- 
earth cation and $\mathrm{B}$ is a transition metal cation in octahedral coordination with oxygen. As shown in Fig. 2-1, the ideal perovskite structure crystallizes in cubic symmetry (space group: Pm3m) with the A cation located at the cube corners and the $\mathrm{BO}_{6}$ octahedra in the center of the cube. The perovskites with different nominal numbers of electrons in their $d$ shells exhibit a variety of physical properties. For example, $\mathrm{LaTiO}_{3}\left(d^{1}\right), \mathrm{LaVO}_{3}\left(d^{2}\right)$ and $\mathrm{LaCrO}_{3}\left(d^{3}\right)$ are found to be Mott insulators accompanied by ferromagnetic or antiferromagnetic order. ${ }^{4}$ Substitution of the trivalent $\mathrm{La}$ ions in $\mathrm{LaMnO}_{3}\left(d^{4}\right)$ with a divalent ion creates a system displaying a combination of metal-insulator transition, ferromagnetic-paramagnetic transition, and colossal magnetoresistance. ${ }^{27}$ Cuprates $\left(d^{9}\right)$ are famous parent compounds for high-temperature superconductors. ${ }^{28}$

The ideal perovskite structure is only stable for very few compounds at room temperature, as $\mathrm{BO}_{6}$ octahedra are often found to be distorted or rotated. The deviation from the ideal structure can be predicted by the Goldschmidt tolerance factor $t=\left(r_{A}+\right.$ $\left.r_{O}\right) / \sqrt{2}\left(r_{B}+r_{O}\right)$ where $r_{A}$ and $r_{B}$ denote the average ionic radii at the $\mathrm{A}$ and $\mathrm{B}$ sites and $r_{O}$ is the radius of the oxygen ion. The quantity $t$ is equal to one when the system is cubic, and the ionic radii $r_{A}, r_{B}$ and $r_{O}$ are derived under the assumption that all the anions just touch the cations. ${ }^{29}$ The $\mathrm{BO}_{6}$ octahedra play a central role in the electrical and magnetic properties of many perovskites. In the cubic perovskite structure, the B-O-B bond angle is $180^{\circ}$. However, when the $\mathrm{A}$ cations are too small, the $\mathrm{BO}_{6}$ octahedra rotates and the tolerance factor becomes less than one. Also, the lattice structure changes to rhombohedral and then to the orthorhombic $\mathrm{GdFeO}_{3}$ type as $r_{A}$ is reduced. ${ }^{30}$ The rotations of the $\mathrm{BO}_{6}$ octahedra lead to the reduction of the $\mathrm{B}-\mathrm{O}-\mathrm{B}$ bond angle thus further affecting 
the overlap between $d$ orbitals and the resulting $d$ bands. This ultimately affects the electronic structures and physical properties. For example, a study of the metallic $\mathrm{Ca}_{1-}$ ${ }_{x} \mathrm{Sr}_{x} \mathrm{VO}_{3}$ system near the metal-insulator transition indicates that the bandwidth can be tuned by the isovalent A-site substitution with a different ionic radius, since the substitution of $\mathrm{Ca}^{2+}$ ions for $\mathrm{Sr}^{2+}$ ions would decrease the $\mathrm{V}-\mathrm{O}-\mathrm{V}$ bond angle from $180^{\circ}$ to $160^{\circ}{ }^{31,32}$

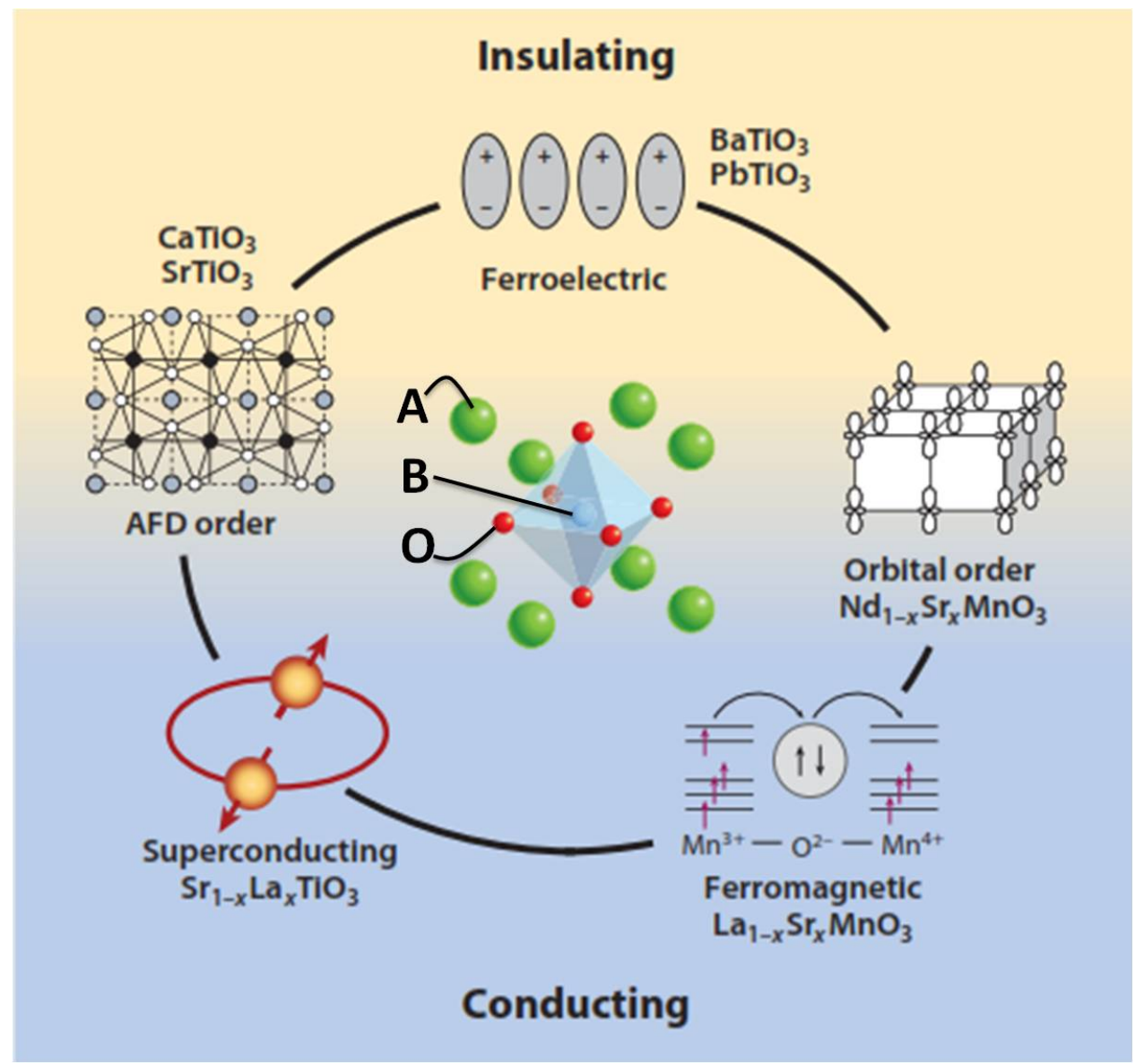

Figure 2-1 The rich functional properties in $\mathrm{ABO}_{3}$ perovskites arising from the complex interplay between spin, charge, orbital and lattice degrees of freedom. The ideal perovskite structure is also shown. (Adapted from Ref. 33) 
In $\mathrm{ABO}_{3}$ perovskites, the complex interactions between spin, charge, orbital and lattice degrees of freedom can be modified by charge transfer, strain, symmetry breaking and electrostatic coupling and frustration. As illustrated in Fig. 2-1, modification of these factors generates a broad spectrum of functional properties for both fundamental and application-oriented research. Such as colossal magnetoresistance and electronic phase transitions caused by charge transport, ferroelectricity or ferromagnetism induced by the cooperative alignment of electric dipoles and spins, and antiferrodistortive (AFD) structural ordering due to the tilting and buckling of oxygen octahedral. ${ }^{33}$ Even more excitingly, complex multifunctional heterostructures can be obtained by stacking the perovskites on top of each other owing to the great structural compatibility. Many exotic phenomena can be found in artificial oxide heterostructures, an important example is the two-dimensional superconductivity obtained at the interface between a Mott insulator $\mathrm{LaTiO}_{3}$ and a band insulator $\mathrm{SrTiO}_{3}{ }^{34}$ Recent advances in thin film oxide growth have opened the door for studies of the fascinating new phenomena at the surfaces and interfaces that are not found in bulk systems. ${ }^{35}$ The fabrication of high quality oxide thin films and heterostructures, and control over the rich properties endowed by the $d$ electrons, will pave the way for making advanced nanoscale devices taking oxide electronics to a new level. 


\subsection{Metal-Insulator Transition}

\subsubsection{Introduction}

The metal-insulator transition (MIT) in the solids refers to the transition from a metallic phase to an insulating phase or vice versa, usually accompanied by huge resistivity changes due to the response to excitations such as temperature, pressure or the doping level. The MIT in TMOs has been one of the major topics in condensed matter physics for decades and has received revived interest since the discovery of hightemperature superconductivity in cuprates and colossal magnetoresistance in manganites. ${ }^{4}$ Studying the transport phenomena in TMOs and unraveling the MIT mechanisms are essential for understanding the electron correlation effects in these materials.

Band theory is successful in describing the electronic structures and predicting the transport properties of materials in which electrons do not strongly interact with each other. ${ }^{36}$ In quantum mechanics, when many atoms form a solid, the available energy levels for electrons form an energy band. The forbidden region between the two bands is called a bandgap. As illustrated in Fig. 2-2, electrons are filled into the energy bands up to the Fermi level. Metals contain a partially filled band while the bands in insulators are either fully occupied or empty. ${ }^{37}$ 


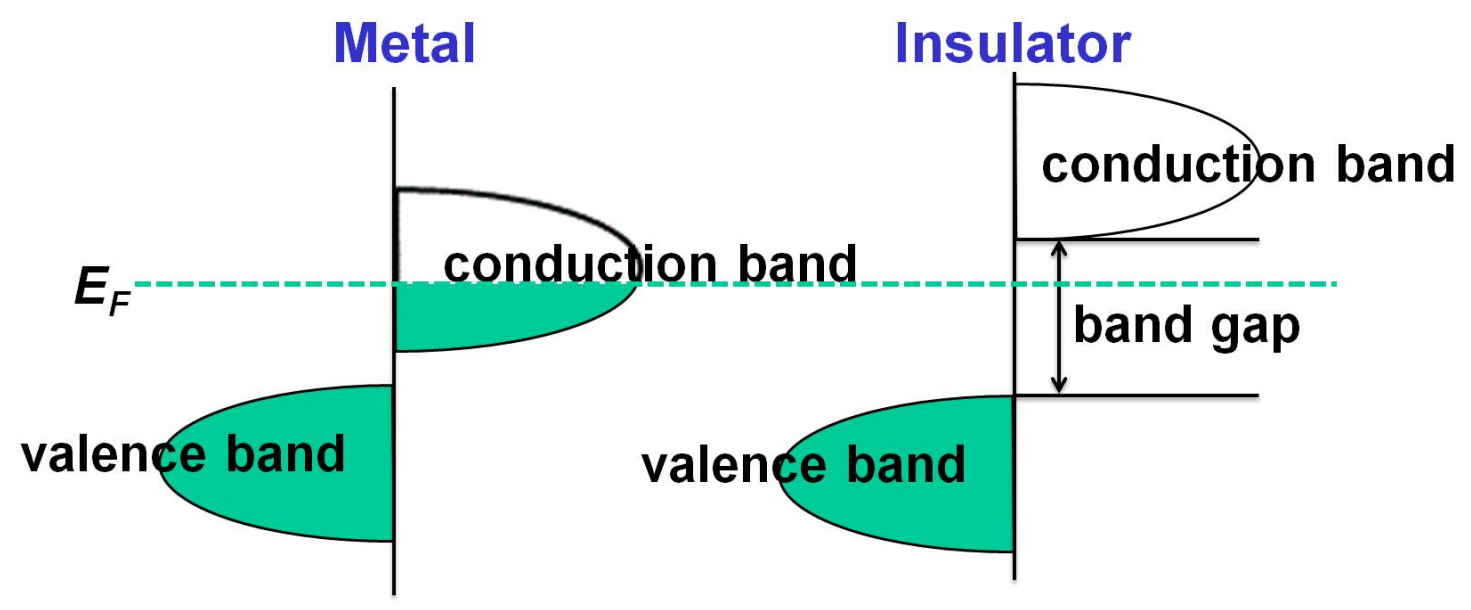

Figure 2-2 Electronic band structures of a metal and an insulator. Fermi level $E_{F}$ is also shown.

Band theory has failed in some TMOs with a partially filled $d$ band that are predicted to be good conductors are in fact insulators. The reason for this disagreement is that the electronic structures of TMOs are affected by strong electron-electron interactions, electron-phonon interactions, and electron-disorder interactions. These effects may modify the transport properties in the TMOs and drive MIT that can be categorized into Mott transition ${ }^{5,6}$, Peierls transition $^{7}$ and Anderson localization ${ }^{8}$, respectively.

\subsubsection{Mott Transition}

Sir N. F. Mott first proposed a model for MIT considering strong electronelectron interactions. ${ }^{5,6}$ The Mott insulating states could be understood from a tightbinding lattice model with one electron per site. Without electron-electron interactions, the single site can accommodate at most two electrons by taking spin degeneracy into 
account. If there is an odd number of electrons, an electron in a singly occupied state can itinerate from one site to another without much energy cost, the material is metallic. However, in strongly correlated systems, when the electron is hopping through the lattice from one site to a new site that is already occupied by another electron, the two electrons residing on the same site experience a large Coulomb repulsion. When the Coulomb repulsion is sufficiently large, double occupancy is prohibited and the electron would be bounded to the original site instead of being itinerant in the lattice and the material becomes a Mott insulator. As shown in Fig. 2-3, under strong electron correlation effects, the single half-filled conduction band splits into a fully filled lower Hubbard band (LHB) formed from electrons occupying an empty site, and an empty upper Hubbard band (UHB) is created from electrons occupying a site already taken by another electron. The bandgap that separates LHB and UHB equals to the magnitude of Coulomb repulsion energy $U$. Such a MIT driven by strong electron-electron interactions is referred as Mott transition.

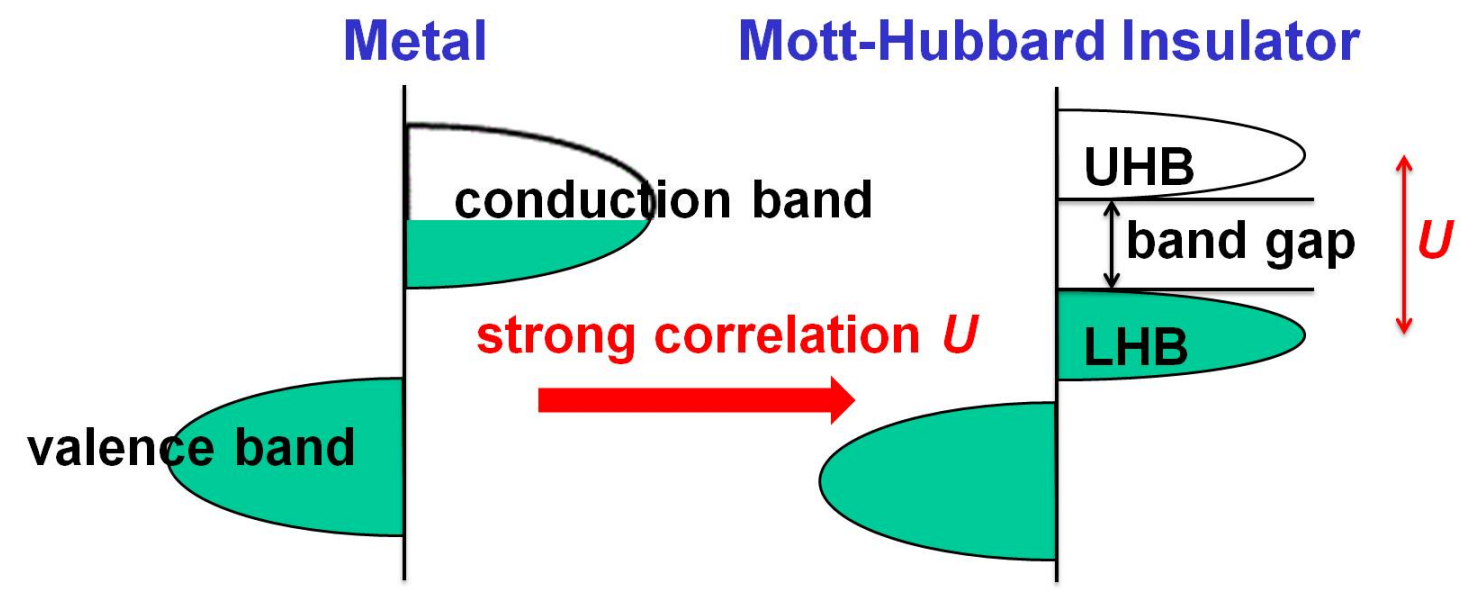

Figure 2-3 Metal-insulator transition driven by strong electron correlation effects. 
The electronic band structures are quite different for TMOs with light transition elements such as $\mathrm{Ti}, \mathrm{V}$, and $\mathrm{Cr}$, compared to those with heavy transition elements such as $\mathrm{Ni}$ and $\mathrm{Cu}$. As the transition element becomes heavier, the $3 d$ band and oxygen $2 p$ band become closer. In heavy TMOs, the $3 d$ band is strongly hybridized with the oxygen $2 p$ band. The difference in the energy separation $\Delta=\left|\varepsilon_{d}-\varepsilon_{p}\right|$ between the $3 d$ band and oxygen $2 p$ band results in two types of Mott insulators: Mott-Hubbard insulators and charge transfer insulators. As depicted in Fig. 2-4 (a) and (b), in the case of a MottHubbard insulator, $\Delta$ is larger than the Coulomb repulsion energy $U$, the oxygen $2 p$ band is much lower than the $3 d$ band, and the bandgap is mainly determined by the Coulomb repulsion energy $U$ from $d$ electrons. In contrast, for a charge transfer insulator, $\Delta$ is smaller than $U$ and the charge excitation in the Mott insulating phase is determined by the charge transfer between the strongly hybridized $3 d$ band and oxygen $2 p$ band. ${ }^{38}$ Fig. 2-4 (c) shows the phase diagram of $\mathrm{ABO}_{3}$ perovskites for $\mathrm{A}=\mathrm{Y}^{3+}, \mathrm{La}^{3+}, \mathrm{Ca}^{2+}$ and $\mathrm{Sr}^{2+}$ as a function of $U / W, \Delta / W$ and electron band filling, where $W$ is the one-electron bandwidth. The thin lines connect A-site cations to B-site cations, and the thick lines represent the solid solutions. Perovskite Mott insulators with light B-site transition elements $\left(d^{1}\right.$ and $\left.d^{2}\right)$ are found to be Mott-Hubbard type, and the ones with heavy B-site transition elements $\left(d^{4}, d^{5}, d^{6}, d^{7}, d^{8}\right)$ are charge transfer type. ${ }^{30}$ 


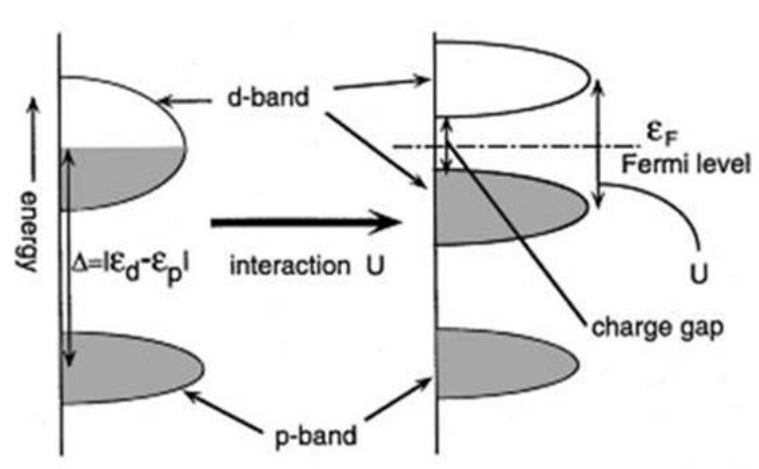

(a) Mott-Hubbard Insulator

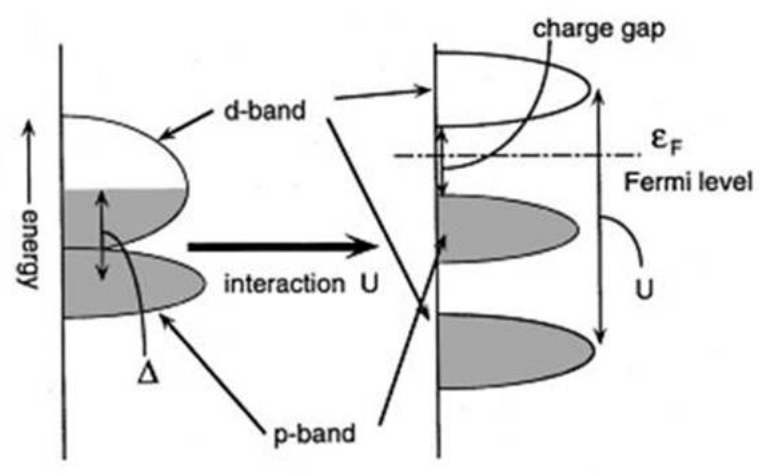

(b) Charge Transfer Insulator

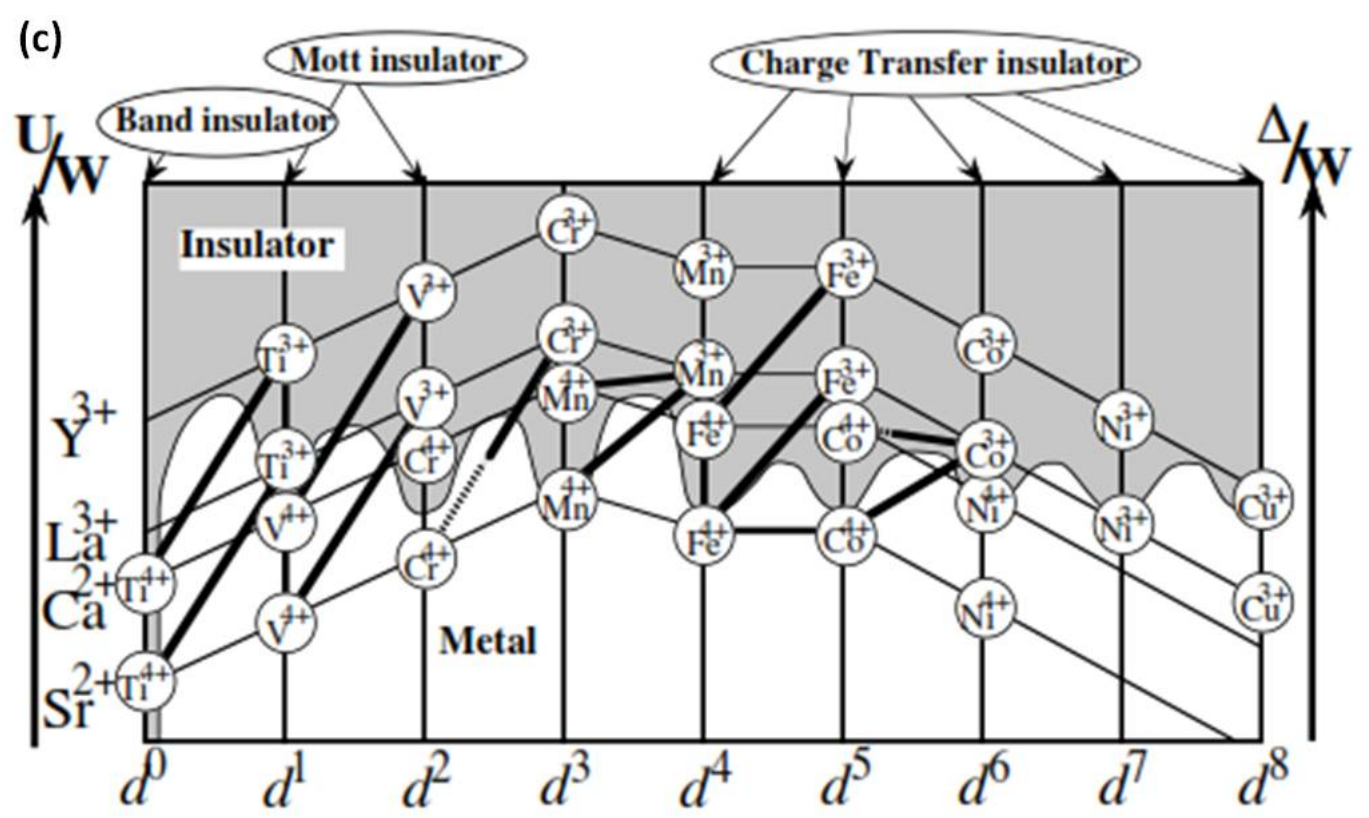

Figure 2-4 Schematic energy levels for (a) Mott-Hubbard insulator and (b) charge transfer insulator. (Adapted from Ref. 4) (c) Schematic phase diagram of $\mathrm{ABO}_{3}$ perovskites for $\mathrm{A}=\mathrm{Y}^{3+}, \mathrm{La}^{3+}, \mathrm{Ca}^{2+}$ and $\mathrm{Sr}^{2+}$ as a function of $U / W, \Delta / W$ and electron band filling. The thin lines connect A-site cations to B-site cations, and the thick lines represent the solid solutions. (Adapted from Ref. 30)

Hubbard took the next important step to understanding the Mott transition by introducing a single band model including only the nearest neighbor hopping $t$ with on- 
site Coulomb repulsion energy $U .^{39}$ In a second quantization, the Hamiltonian for this model is:

$$
H=-t \sum_{<i, j>} c_{i \sigma}^{\dagger} c_{j \sigma}+U \sum_{i} n_{i \uparrow} n_{i \downarrow}-\mu \sum_{i \sigma} n_{i \sigma}
$$

The first term is the kinetic energy of the electrons, where $c_{i \sigma}^{\dagger}\left(c_{i \sigma}\right)$ is the creation (annihilation) operator of the single band electron of spin $\sigma$ at site $i$, the suffixes $i$ and $j$ refer to that the hopping is only allowed between the nearest-neighbor sites. The second term is the on-site Coulomb repulsion energy. The final term is the chemical potential that controls the band filling, where $n_{i \sigma}$ is the electron number operator $n_{i \sigma} \equiv c_{i \sigma}^{\dagger} c_{i \sigma}$. In this model, the electron band filling $n$ and the ratio $U / W$ between the Coulomb repulsion energy $U$ and the one-electron bandwidth $W$ turn out to be very critical parameters that can drive MITs.

According to the Hubbard model, the control parameters for a Mott transition can be categorized into bandwidth control and band-filling control. ${ }^{4}$ These two routes across the MIT are depicted in Fig. 2-5 (a). In the bandwidth-controlled MIT (BC-MIT), the system is determined by the competition between the on-site Coulomb repulsion $U$ (localizes the electron), and the one-electron bandwidth $W$ (the tendency of electrons to delocalize). A system with small $U / W$ (wide bandwidth $W$ ) corresponds to a metallic phase in which the fully occupied LHB and the unoccupied UHB overlap with each other. As the bandwidth $W$ decreases, the splitting between LHB and UHB gradually increases, eventually evolves into a bandgap at the Fermi level when $U / W$ is beyond a critical value $(U / W)_{\mathrm{c}}$ and the system becomes a Mott insulator. In the filling-controlled MIT (FC-MIT), 
doping the UHB of a Mott insulator (filling $n=1$ ) with electrons or doping the LHB with holes results in non-integer $n$ and a transition to a metallic state. Fig. 2-5 (b) shows the metal-insulator phase diagram of some perovskite titanates and vanadates. $\mathrm{CaTiO}_{3}$ and $\mathrm{SrTiO}_{3}$ with $n=0$ are band insulators, $\mathrm{YVO}_{3}$ and $\mathrm{LaVO}_{3}$ with $n=2$ are Mott insulators. The BC-MIT is demonstrated in the perovskites with $n=1$, the vanadates $\left(\mathrm{VO}_{2}, \mathrm{SrVO}_{3}\right.$, and $\left.\mathrm{CaVO}_{3}\right)$ are metals, whereas the titanates $\left(\mathrm{LaTiO}_{3}\right.$ and $\left.\mathrm{YTiO}_{3}\right)$ with a higher $U / W$ value are insulators. The FC-MIT can be induced via aliovalent A-site substitution, such as in the $(\mathrm{Ca}, \mathrm{Y}) \mathrm{VO}_{3}$ and $(\mathrm{Sr}, \mathrm{La}) \mathrm{VO}_{3}$ solid solution systems. ${ }^{40}$
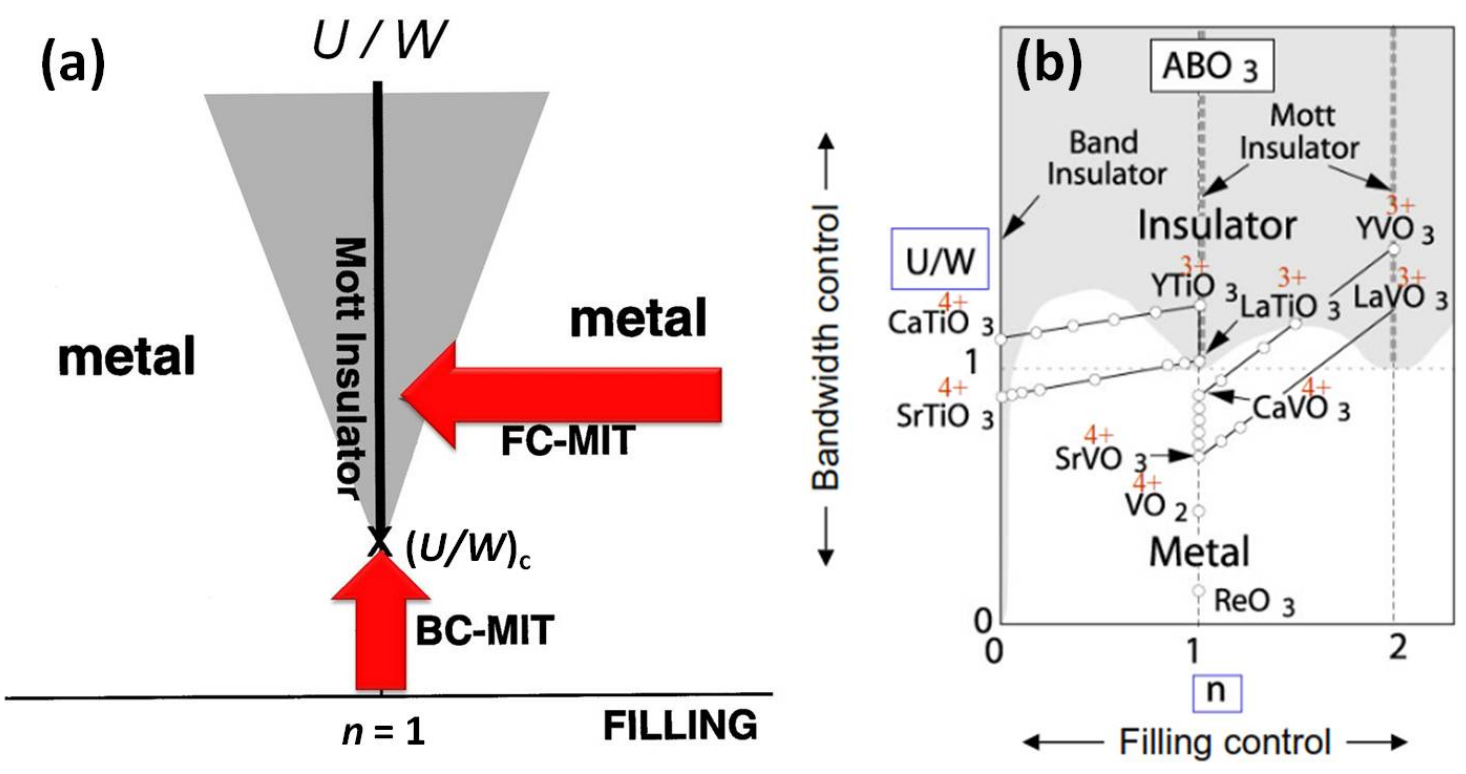

Figure 2-5 (a) Bandwidth-controlled MIT (BC-MIT) and fillingcontrolled MIT (FC-MIT) in a Mott-Hubbard system. (Reproduced from Ref. 4) (b) Metal-insulator phase diagram of some perovskite titanates and vanadates as a function of $U / W$ and electron band filling. (Adapted from Ref. 40) 


\subsubsection{Peierls Transition}

A Peierls transition is the MIT arising from electron-phonon (electron-lattice) interactions. ${ }^{7}$ Fig. 2-6 (a) and (c) show a one-dimensional metal with lattice constant $a$ and its band structure. The conduction band is partially filled. The value $k_{F}$ refers to the Fermi wave vector of the metal. If a distortion happens and the periodicity of the lattice deformation is $\lambda=\pi / k_{F}$ as shown in Fig. 2-6 (b), a new Brillouin-zone boundary will coincide with the Fermi level of the unperturbed lattice, thereby opening a bandgap at the Fermi level. The band opening lowers the energy of electrons below the gap and destroys the metallic properties of the conduction electrons (Fig. 2-6 (d)). Such periodic modulation of lattice is also known as a charge-density-wave (CDW). ${ }^{41}$

(a)

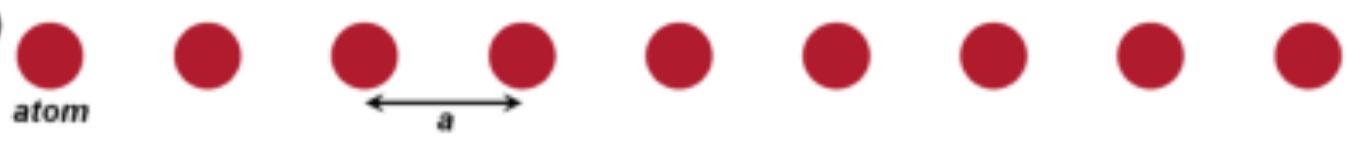

(b)

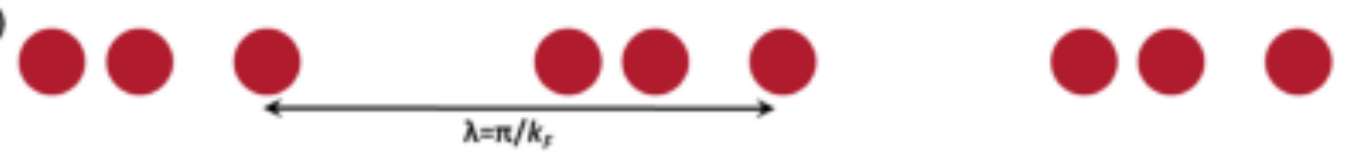

(c)

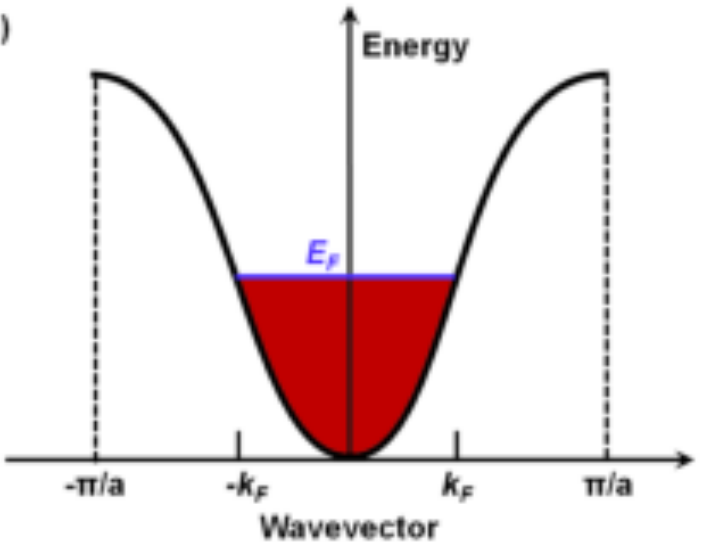

(d)

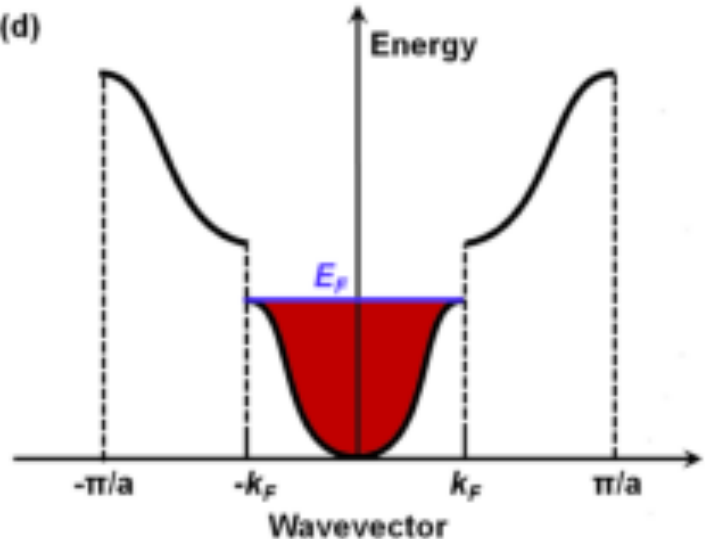

Figure 2-6 (a) One-dimensional metal with lattice constant $a$ and (b) Peierls insulator with the periodic modulation (charge-density-wave) $\lambda=\pi / k_{F}$ and their band structures (c) and (d). (Adapted from Ref. 42) 
Peierls predicted that a one-dimensional metal is susceptible to be in a CDW state and could not exist at $\mathrm{T}=0 \mathrm{~K}$, since the reduction in the energy of electrons from the band opening is always larger than the gain in the elastic energy. ${ }^{7,43}$ The energy reduction from electrons becomes smaller at high temperatures, therefore the undistorted metal would be energetically favorable at a critical temperature at which the Peierls insulator becomes a metal. The Peierls transition is a common phenomenon in quasi-onedimensional systems, such as $\mathrm{NbSe}_{3}{ }^{44}$ and $\mathrm{K}_{0.30} \mathrm{MoO}_{3}{ }^{45}$. In these materials, atoms form parallel chains that are responsible for the electrical conductivity. The interchain interactions are very weak, their band structures are quasi-one-dimensional, and the atomic orbitals forming the conduction band have an appreciable overlap in one direction and much smaller overlaps in the perpendicular directions. ${ }^{46}$

\subsubsection{Anderson Localization}

Structural and chemical imperfections are inevitable in crystals. Defects causing lattice disorder, such as impurities and vacancies, play a substantial role in the transport properties of electronic materials. Anderson localization predicts that an electron may become immobile in the presence of strong lattice disorder. ${ }^{8,47,48}$ The origin of Anderson localization is the interference between multiple scattering of the electron by random defects, resulting in either localized or extended electronic states for a given energy in a disordered system. The electronic states close to the band edges are localized and the envelope of the wave functions is characterized by:

$$
|\psi(\boldsymbol{r})| \sim \exp \left(\left|\boldsymbol{r}-\boldsymbol{r}_{\mathbf{0}}\right| / \xi\right)
$$


where $\xi$ is the localization length. ${ }^{49}$ Fig. $2-7$ shows the mobility edges $E_{c}$ separating the localized states from extended states. The locations of the mobility edges depend on the degree of disorder. As disorder increases, more electronic states become localized and the mobility edges move toward the center of the band. When the Fermi level lies in the extended states, the system is a metal. On the other hand, when the Fermi level lies in the localized states, the system becomes an Anderson insulator. Therefore, a MIT could happen as the Fermi level passes through a mobility edge. ${ }^{49}$

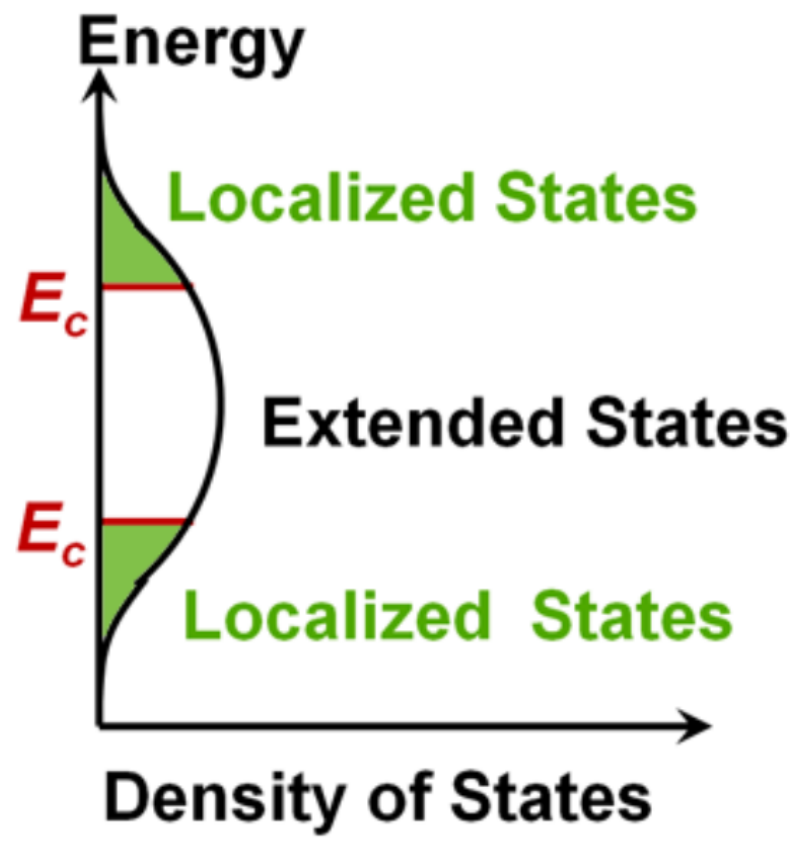

Figure 2-7 Schematic density of states in a disordered system, showing mobility edges separating localized and extended states. (Adapted from Ref. 42 ) 


\subsection{Perovskite Vanadates: $\mathrm{CaVO}_{3}$ and $\mathrm{SrVO}_{3}$}

\subsubsection{Introduction}

Perovskite vanadates $\mathrm{CaVO}_{3}(\mathrm{CVO})$ and $\mathrm{SrVO}_{3}(\mathrm{SVO})$ with the $3 d^{1}$ electronic configuration for vanadium are typical strongly correlated systems. Both CVO and SVO are paramagnetic metals, with CVO being closest to a Mott transition. Their physical properties, especially the transport properties in thin film CVO and SVO, remain largely unexplored due to the great challenges in the synthesis of stoichiometric vandadates.

\subsection{2 $\mathrm{CaVO}_{3}$}

$\mathrm{CaVO}_{3}(\mathrm{CVO})$ crystallizes in the orthorhombically distorted $\mathrm{GdFeO}_{3}$-type structure (space group: Pbnm) with lattice parameters a $=5.3219 \AA, \mathrm{b}=5.3427 \AA$, and c $=7.5472 \AA$, and each unit cell can be viewed as four pseudo-cubic perovskite cells with a lattice parameter of $3.77 \AA .^{50}$ As shown in Fig. $2-8$, owing to the relatively small radius of A-site cation, the $\mathrm{VO}_{6}$ octahedra rotates, and the $\mathrm{V}-\mathrm{O}-\mathrm{V}$ bond angle is $\sim 160^{\circ} .31,32$
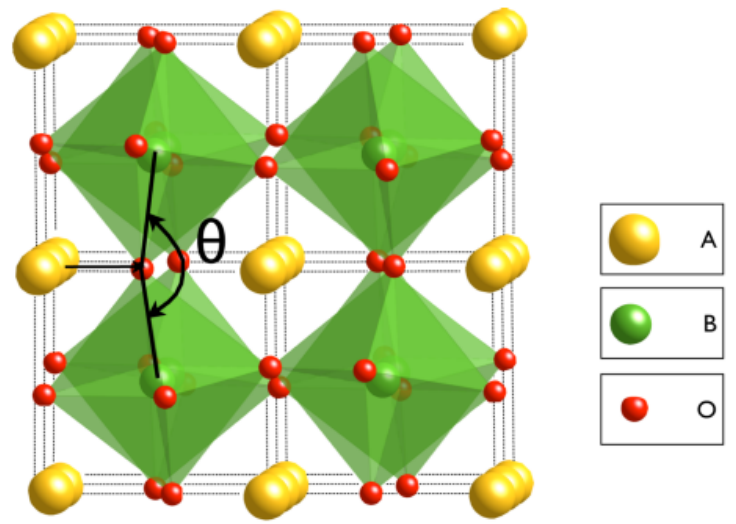

Figure 2-8 Orthorhombically distorted $\mathrm{GdFeO}_{3}$-type structure, $\theta$ is the $\mathrm{B}$ O-B bond angle. (Adapted from Ref. 51) 
Bulk CVO has been found to be a paramagnetic metal. ${ }^{26}$ It has also been reported that the crystal microstructure and oxygen stoichiometry play an important role in the electrical and magnetic properties of CVO. For example, small changes in the oxygen content resulted in a much higher residual resistivity or even semiconducting behavior, ${ }^{52,53}$ and the oxygen-deficient phases yielded Curie-Weiss behavior. ${ }^{54,55}$ Fig. 2-9 (a) shows the temperature dependence of the electrical resistivity for single-crystalline CVO. Oxygen off-stoichiometry caused an great increase in residual resistivity $\rho_{0}$, which varied from $10^{-6}$ to $10^{-3} \Omega \mathrm{cm} .{ }^{52}$ Stoichiometric CVO thin films were first synthesized by pulsed laser deposition in 2009. As shown in Fig. 2-9 (b), the transport measurements of the $45 \mathrm{~nm}$ CVO films grown on different substrates revealed metallic behavior with the electrical resistivity comparable with bulk CVO. The temperature dependence of the electrical resistivity followed a $\rho=\rho_{0}+A T^{2}$ fit corresponding to a Fermi liquid model. ${ }^{56}$
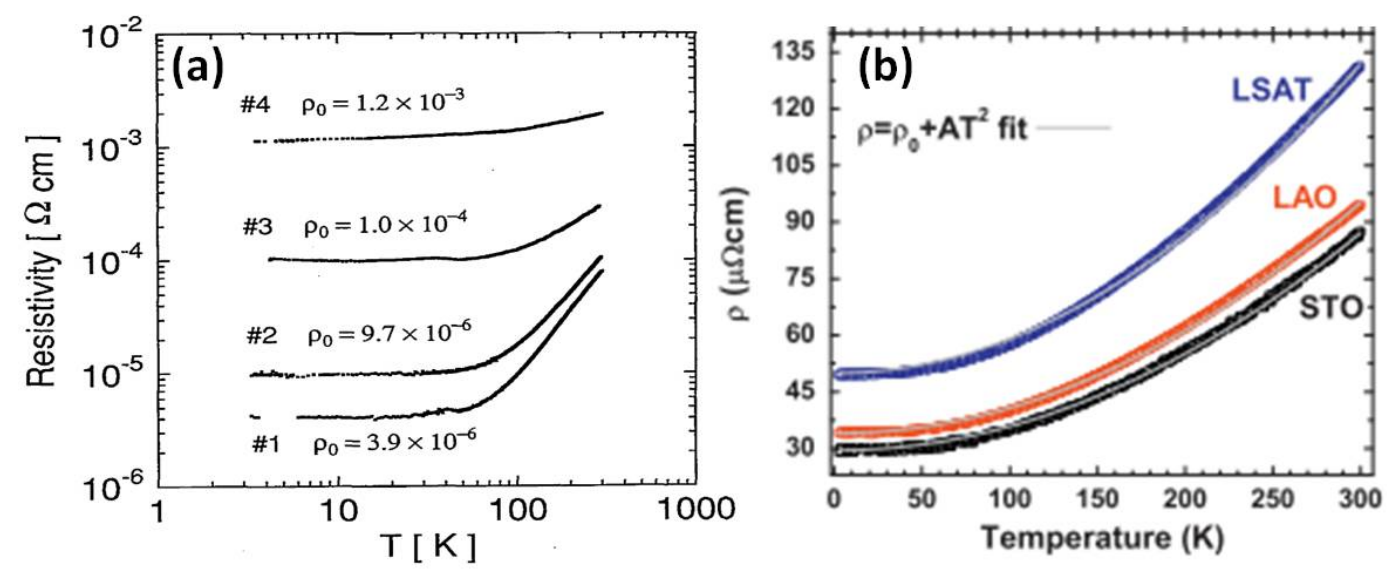

Figure 2-9 Electrical resistivity vs. temperature of (a) single-crystalline $\mathrm{CVO}$ with increasing oxygen off-stoichiometry. (Adapted from Ref. 52) (b) $45 \mathrm{~nm}$ CVO films grown on $\mathrm{SrTiO}_{3}(\mathrm{STO}),\left(\mathrm{LaAlO}_{3}\right)_{0.3}\left(\mathrm{Sr}_{2} \mathrm{AlTaO}_{6}\right)_{0.7}$ (LSAT) and $\mathrm{LaAlO}_{3}$ (LAO) substrates. A $\rho=\rho_{0}+A T^{2}$ fit is also shown. (Adapted from Ref. 56) 


\subsection{3 $\mathrm{SrVO}_{3}$}

$\mathrm{SrVO}_{3}(\mathrm{SVO})$ crystallizes in a cubic perovskite structure (space group: $P m 3 m$ ) with a lattice constant of $3.843 \AA{ }^{57}$ Bulk SVO has been reported to be metallic with electrical resistivity ranging from $10^{-5}$ to $10^{-3} \Omega \mathrm{cm}$ at room temperature. ${ }^{57,26,58,59}$ Recently, SVO thin films have been investigated as high conductivity electrodes, and all the transport studies showed good metallic behavior comparable with bulk SVO. ${ }^{60,61,62,63}$ For example, Fig. 2-10 shows the temperature dependence of electrical resistivity for $10 \mathrm{~nm}$ SVO films grown on different substrates. All of the films exhibited metallic behavior with electrical resistivity around $10^{-4} \Omega \mathrm{cm}$ in the temperature range of $30 \mathrm{~K}-280 \mathrm{~K}^{62}$

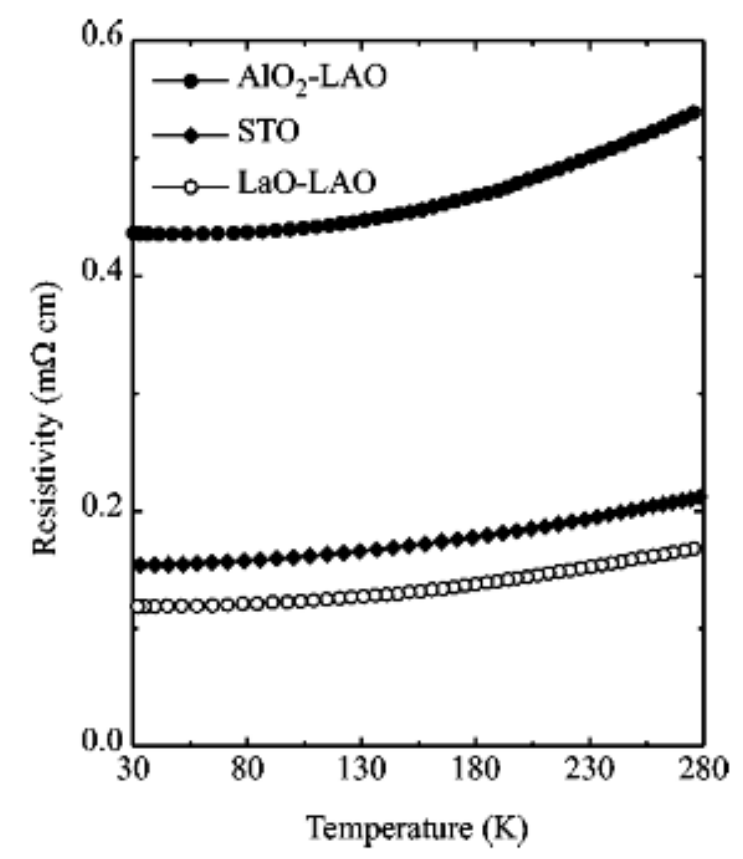

Figure 2-10 Electrical resistivity vs. temperature of $10 \mathrm{~nm}$ SVO films grown on $\mathrm{AlO}_{2}$-terminated $\mathrm{LaAlO}_{3}\left(\mathrm{AlO}_{2}\right.$-LAO), $\mathrm{SrTiO}_{3}(\mathrm{STO}), \mathrm{LaO}-$ terminated $\mathrm{LaAlO}_{3}(\mathrm{LaO}-\mathrm{LAO})$ substrates. (Adapted from Ref. 62) 


\subsubsection{MITs Induced in $\mathrm{CaVO}_{3}$ and $\mathrm{SrVO}_{3}$}

Bulk CVO and SVO are in the metallic regime of the MIT. Most previous studies of MIT in these systems were achieved by chemical substitution. According to the Hubbard model, the MIT can be controlled by modifying the band filling or the bandwidth. It has been reported that FC-MITs can be induced in bulk CVO and SVO via aliovalent A-site substitution, such as in the $\mathrm{La}_{1-x} \mathrm{Ca}_{x} \mathrm{VO}_{3}{ }^{64,65}, \mathrm{Gd}_{1-x} \mathrm{Ca}_{x} \mathrm{VO}_{3}{ }^{66}$ and $\mathrm{La}_{1-}$ ${ }_{x} \mathrm{Sr}_{x} \mathrm{VO}_{3}{ }^{67,68,69}$ systems. Also, the study of the metallic $\mathrm{Ca}_{1-x} \mathrm{Sr}_{x} \mathrm{VO}_{3}$ system near the MIT has shown that the bandwidth can be tuned by isovalent A-site substitution with different ionic radius. ${ }^{31,32}$ However, chemical substitution would unavoidably introduce significant disorder into the system, making it difficult to isolate the purely electronic contributions from the chemical disorder effects.

In recent years, major improvements in thin-film correlated oxides synthesis techniques have provided new methods to study the MIT in ways not possible with bulk materials. Novel phenomena and functionalities can arise from strong electron interactions by scaling down the dimensions of the thin film. Low dimensional SVO ultrathin films have been studied recently using in situ photoemission spectroscopy (PES), ${ }^{70,71,72}$ and the evolution of the electronic structure at Fermi energy $E_{F}$ revealed a dimensional-crossover-driven MIT. As shown in Fig. 2-11, in the case of thicker SVO films, the PES V $3 d$ spectra consist of the coherent part (the quasi-particle band) located at $E_{F}$, and the incoherent part (the remnant of the LHB). As the film thickness decreased, a bandgap was formed at $E_{F}$ due to a transfer of spectral weight from the coherent to the incoherent part, indicating a MIT at a critical film thickness of 2-3 monolayers (ML). 
Dynamical-mean-field-theory (DMFT) calculations in this work also indicated the Coulomb repulsion $U$ remained unchanged with a decrease in the SVO film thickness, and the observed Mott insulating states can be understood by the reduction in the bandwidth $W$ due to the dimensional crossover. ${ }^{70}$
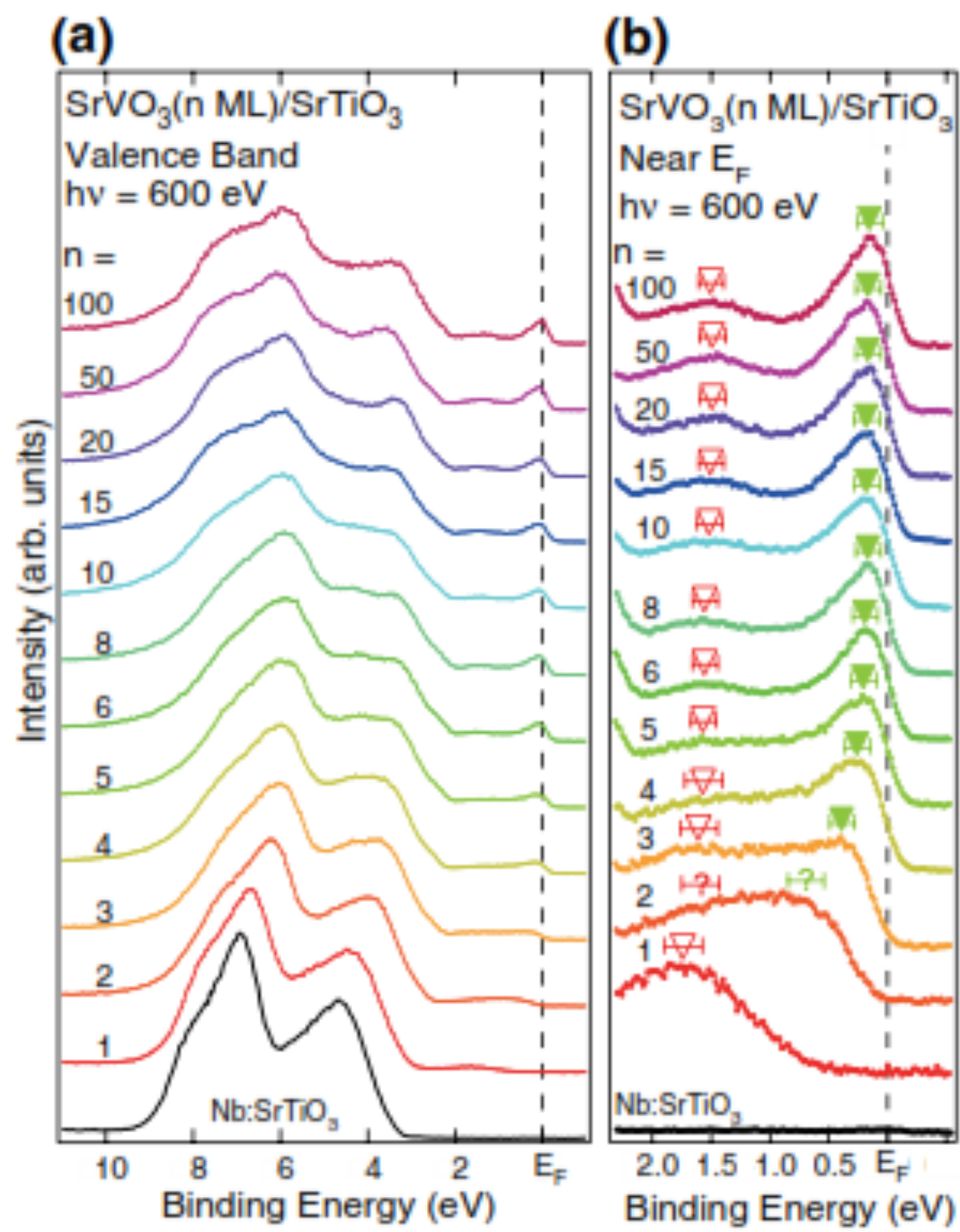

Figure 2-11 (a) In situ valence band spectra of SVO films with thicknesses from 1 to $100 \mathrm{ML}$. (b) PES spectra of the same SVO films near $E_{F}$. The solid and opened triangles indicate the energy positions of the coherent and incoherent parts, respectively. (Adapted from Ref. 70) 


\subsection{Thin Film Growth}

Complex oxide thin films have been investigated by numerous growth techniques namely physical vapor deposition (PVD) and chemical vapor deposition (CVD) ${ }^{73}$ Common PVD techniques include evaporation, molecular beam epitaxy (MBE), pulsed laser deposition (PLD), and sputtering. PLD is a widely used approach to complex oxide thin film growth owing to its great ability to retain the target composition in the corresponding film. A variety of stoichiometric oxide thin films have been grown in either an inert or a reactive oxygen background atmosphere without the need for further

processing. ${ }^{74,75,76}$ In this process, a pulsed highly energetic laser beam is focused onto the surface of a target in order to get a sufficiently large energy density for ablation of the target material. The vaporized material ablated from the target then forms a plasma plume, which is a mixture of atoms, molecules, ions and electrons propagating in a direction perpendicular to the target surface. The material finally condenses on a heated substrate placed in front of the target, forming a thin film. The stoichiometric transfer of the material from a multi-cation target arises from the non-equilibrium nature of the ablation process itself. The high energy density of the laser is absorbed by a small volume of material, leading to rapid vaporization that is not dependent on the vapor pressures of the constituent elements of the target allowing for stoichiometric deposition.

\subsubsection{Pulsed Electron-Beam Deposition Procedures}

In recent years, a novel pulsed electron-beam deposition (PED) tool has emerged as a promising alternative to PLD. Instead of an expensive complex laser source, a high 
power pulsed electron-beam source (PEBS) is employed to ablate the target. Additionally, compared with other growth techniques, PED does not require dangerous gases, has a smaller footprint, and are more ready for high volume manufacturing processes. PED utilizes a channel-spark discharge to produce a pulsed electron beam that propagates in a self-focused way due to the space-charge neutralization. ${ }^{77}$ As illustrated in Fig. 2-12, the channel-spark mechanism involves a transient hollow cathode to produce a high energy electron beam, a triggering source for ignition, a dielectric channel to guide the electron beam to the target in the vacuum chamber. ${ }^{78}$

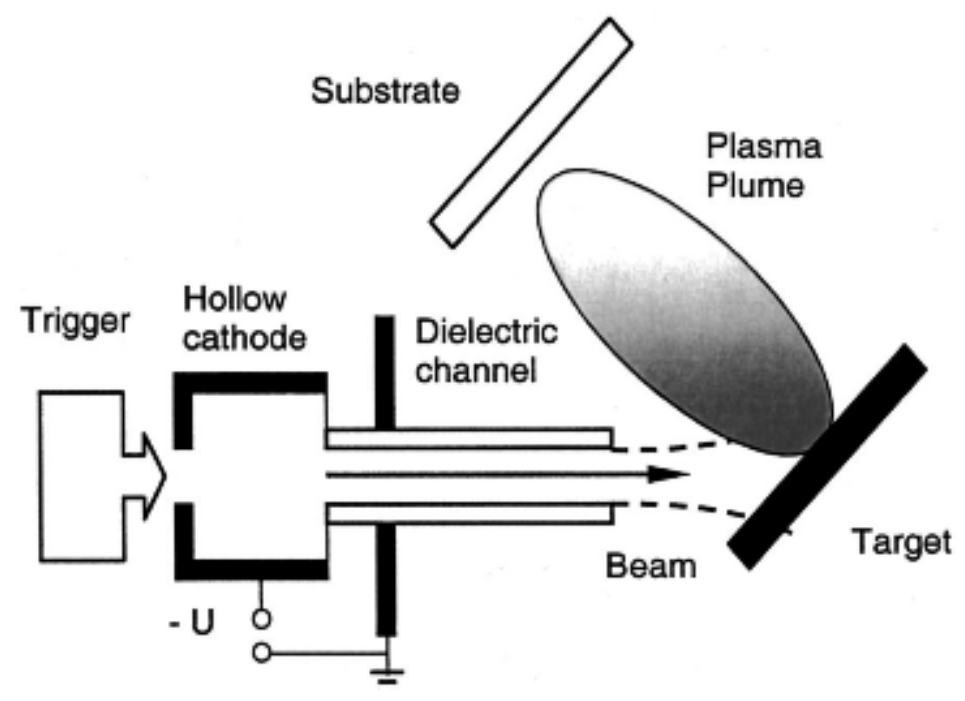

Figure 2-12 General Scheme of PED process. (Adapted from Ref. 78)

In the PED process - which is conceptually similar to PLD - the electron pulses with energies on the order of a Joule and pulse durations of $\sim 100$ ns penetrate approximately $1 \mu \mathrm{m}$ into the target and cause rapid evaporation due to non-equilibrium heating, leading to the stoichiometric transfer of the target composition in the deposited 
film under optimal conditions. The PEBS used in our experiments is a commercial source, PEBS-20 manufactured by Neocera, Inc, and the technical specification is listed in Table 2-1. In PLD, having a similar power density of $\sim 10^{8} \mathrm{~W} / \mathrm{cm}^{2}$, the ablation is critically dependent on the optical absorption coefficient of the target material. Unlike in PLD, the mechanism of interaction with the target material in PED is governed by electrons. Thus, the energy transfer into the target surface is more effective in PED than in PLD and as a result it is possible to process wide band gap materials and materials that are transparent or highly reflective to laser light. ${ }^{79}$

Table 2-1 Specification of Neocera PEBS-20. (Adapted from Ref. 80)

\begin{tabular}{|l|l|}
\hline Input voltage & $115-230 \mathrm{VAC}, 50 / 60 \mathrm{~Hz}$, single phase \\
\hline Gas pressure, oxygen & $5-20 \mathrm{mTorr}$ \\
\hline Energy of electrons & $8-20 \mathrm{keV}$ \\
\hline Single pulse energy & $0.1-0.8 \mathrm{~J}$ \\
\hline Pulse energy variation, maximum & $\pm 10 \%$ \\
\hline Energy conversion efficiency & $25-30 \%$ \\
\hline Pulse width & $\sim 100 \mathrm{~ns}$ \\
\hline Pulse repetition rate, maximum & $15 \mathrm{~Hz}$ \\
\hline Beam cross section, maximum & $6 \times 10^{-2} \mathrm{~cm}^{2}$ \\
\hline Beam cross section variation, maximum & $\pm 20 \%$ \\
\hline Pulse power density, maximum & $1.3 \times 10^{8} \mathrm{~W} / \mathrm{cm}^{2}$ \\
\hline Z alignment range & $50 \mathrm{~mm}$ \\
\hline XY alignment range & $\pm 20 \mathrm{~mm}$ \\
\hline Cathode module lifetime & $10^{7} \mathrm{pulses}$ \\
\hline Temperature of PEBS body, maximum & $85^{\circ} \mathrm{C}\left(180^{\circ} \mathrm{F}\right)$ \\
\hline
\end{tabular}


The PED process can be divided into the following stages: electron beam-material interaction, plasma plume expansion, nucleation and growth of a thin film on the substrate. Each of these steps plays an important role in the composition, crystalline quality and surface morphology of the resulting film.

In the electron beam-material interaction stage, at sufficiently high electron flux power density that is beyond the ablation threshold of the target material, the high-power electron pulse can become strongly coupled to the target material. This leads to an immediate rise in the surface temperature and eventual vaporization of the target material that is not dependent on the vapor pressures of the constituent cations or sub-oxides. Therefore, the material is ablated out with the same stoichiometry as in the target. The ablation threshold $Q$ is the minimal absorbed power density required, and can be expressed by the rate of the surface temperature rise:

$$
d T / d t \approx Q\left[C \rho\left(D+D_{T}\right)\right]^{-1}=I U\left[S C \rho\left(D+D_{T}\right)\right]^{-1}
$$

where $C$ is the specific heat capacity, $\rho$ is the target density. The ablation is highly dependent on the power carried by the electron beam focused onto the target surface: $Q S \approx I U$, where $I$ is the beam current, $U$ is the discharge voltage and $S$ is the beam crosssection on the target surface. The electron pulse energy is distributed over a depth of $D+D_{T}$, where $D$ is the absorption length (electron range) that is found to be proportional to $U^{2}$, and $D_{T}$ is the thermal diffusion length. ${ }^{78}$ The major drawback to the pulsed energy deposition method is the formation of particulates at the film surface, since the highenergy irradiation may cause the ejection of molten particulates from the target that can 
be incorporated in the resulting film. In the PED process, it has been demonstrated that a decrease in the electron beam energy leads to a drastic reduction in the density and size of particulates at the surface of the films. ${ }^{81}$ Therefore, a careful optimization of the electron beam parameters is crucial to achieving high quality epitaxial thin films. Additionally, target selection can significantly impact the film quality. Polycrystalline ceramic targets are the most popular choice for the complex oxide thin film growth. The target materials are preferred to be highly dense as this tends to reduce the particulate formation in the ablation process.

Ejected material expands rapidly into a plasma plume in the vicinity of the target surface, propagating in the direction normal to the target into the background gas towards the substrate. The dynamic expansion of the plasma plume depends on the background gas pressure inside the PED chamber. Since the background gas atoms scatter, decelerate and thermalize the plasma plume, changing the background gas pressure will change the spatial and energy distribution of the depositing species. In general, raising the background gas pressure slows down the high-energy species in the expanding plasma plume. This can strongly influence the film quality since the plasma species with sufficiently high energies may cause material to be resputtered from the film surface leading to a reduction in the effective growth rate and even a change in the film composition and morphology. Modeling of the plasma expansion also indicates that the plume range $L$, which is close to the optimal substrate to target distance, strongly depends on the background gas pressure $P$ as $L \sim(N / P)^{1 / 3}$, where $N$ is the number of atoms propagating within a solid angle..$^{78,82}$ 
During the film nucleation and growth stage, the plasma species adsorbed onto a substrate surface may diffuse over some distance before they interact among themselves and start to nucleate. The nucleation step is crucial as it governs the crystallinity and morphology of the film. In general, there are three basic modes of nucleation and growth: (a) layer-by-layer (Frank-Van der Merwe) growth (b) island (Volmer-Weber) growth, and (c) layer-plus-island (Stranski-Krastonov) growth. In the layer-by-layer growth, the deposited species form a full monolayer before significant clusters are developed on the next film layer. Step-flow growth is a unique layer-by-layer growth induced by the substrate misorientation (off-cut angle), whereby the arriving species migrate to the step edges that are preferential binding sites. In the island growth, the species being deposited are more strongly bonded to each other than to the substrate material, forming individual islands that are multiple atomic layers in height. Stranski-Krastonov growth occurs when the initial layer-by-layer growth changes to island growth after a few monolayers due to the relaxation of strain caused by the lattice mismatch between the film and substrate. ${ }^{73} \mathrm{~A}$ schematic of the growth modes mentioned above is shown in Fig. 2-13. The selection of one of these growth modes is determined by the surface energies of the film and substrate, and the interface energy between the film and substrate. The thin film growth is controlled by the interplay of thermodynamics and kinetics. Therefore, the substrate temperature plays an important role in achieving a high quality epitaxial film since an increase in the substrate temperature will increase the mobility of the deposited species at the substrate surface, thereby allowing them to move to more energetically favorable sites. Moreover, the optimum substrate temperature for film growth is also affected by the 
deposition rate: the higher the deposition rate, the higher the substrate temperature must be for the production of epitaxial thin films. ${ }^{83}$

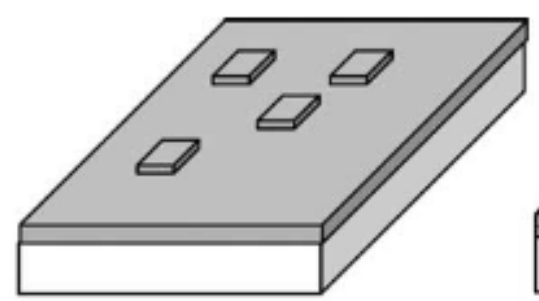

layer-by-layer

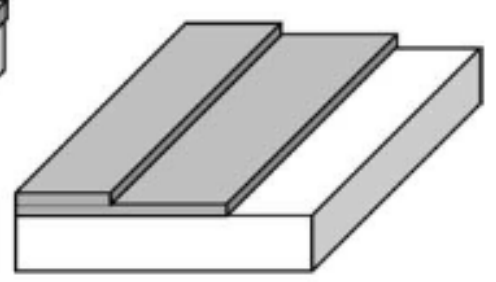

step-flow
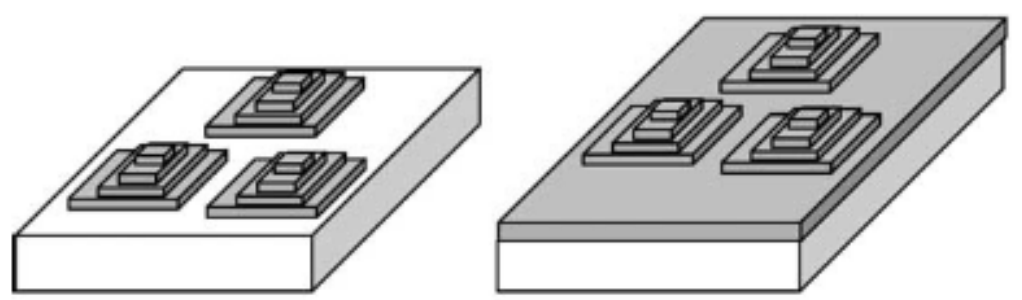

island

\section{Stranski-Krastanov}

Figure 2-13 Schematic illustration of various growth modes in thin film growth. (Adapted from Ref. 74)

PED has been proven to be capable of growing complex oxide thin films such as high temperature superconductors (YBCO, LSCO) and wide band gap semiconductors $\left(\mathrm{SnO}_{2}, \mathrm{ZnO}\right) .{ }^{84,85,86,87}$ However, as a novel thin film growth technique, the capabilities of PED are not fully explored due to the rather complex deposition process. In order to achieve high quality epitaxial thin films, it is important to optimize the key parameters in an adequate manner, such as the electron-beam parameters, the background gas pressure and the substrate temperature. We have demonstrated approaches for optimizing the film 
growth conditions in the PED process to produce epitaxial thin films of comparable quality to those grown by PLD or MBE. ${ }^{88}$

\subsubsection{Role of Substrate}

Substrates play a critical role in the growth of epitaxial thin films, the factors govern the choice of substrate include film/substrate lattice match, thermal expansion match and chemical compatibility. Single crystal oxide substrates with a square-planar surface orientation, such as the (001) face of a cubic or tetragonal crystal structure, are ideal for the growth of $c$-axis oriented perovskite oxide thin films.

Epitaxy can be classified into two major types: homoepitaxy and heteroepitaxy. Homoepitaxy refers to the growth of a film of the same material as the substrate, while on the other hand, heteroepitaxy refers to the case where the film and substrate are composed of different materials. As illustrated in Fig. 2-14, the lattice mismatch between the two materials gives rise to an epitaxial strain as the in-plane film lattice contracts or expands to accommodate the lattice structure of the substrate. Generally, the epitaxial strain is elastic: the in-plane lattice deformation results in an out-of-plane lattice deformation. The lattice mismatch is given by:

$$
f=\frac{2\left(a_{f}-a_{s}\right)}{a_{f}+a_{s}} \sim \frac{a_{f}-a_{s}}{a_{f}}
$$

where $a_{f}$ is the bulk lattice parameter of the film material, and $a_{s}$ is the lattice parameter of the substrate. ${ }^{89}$ In the case of $a_{f}>a_{s}$, the epitaxial strain is expected to be compressive as the film lattice is in-plane compressed and out-of-plane elongated. 
Otherwise, when $a_{f}<a_{s}$, an in-plane tensile strain is induced in the deposited film, and the film lattice is out-of-plane compressed.

(a)
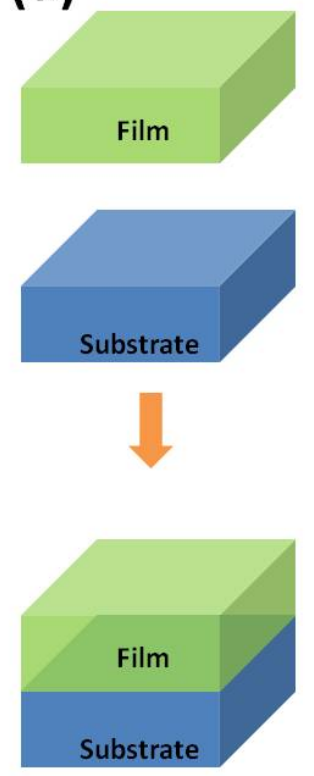

No Strain (b)
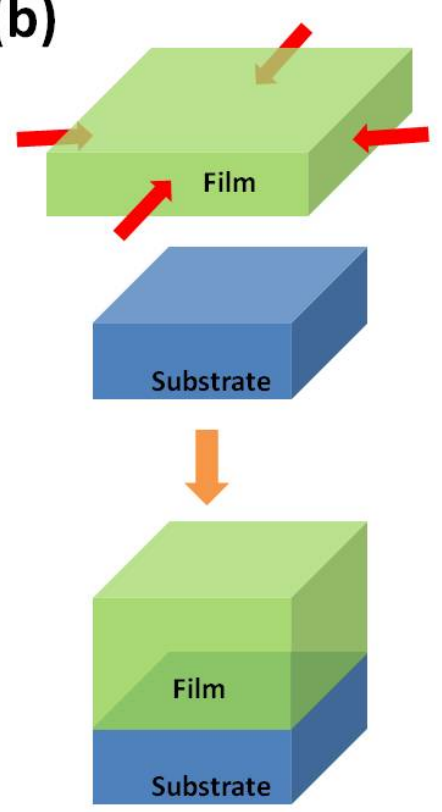

Compressive Strain (c)
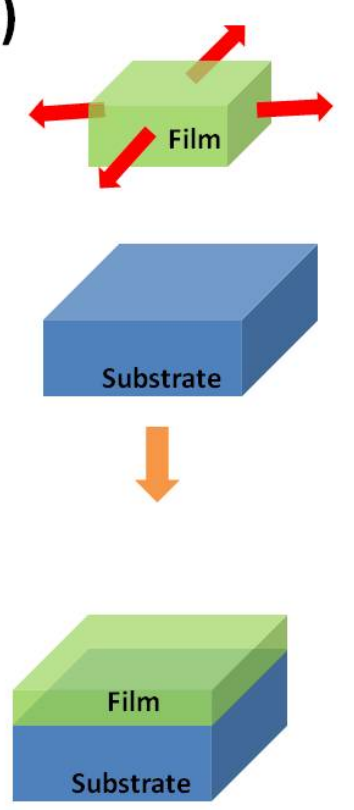

Tensile Strain

Figure 2-14 Schematic view of epitaxial strain: (a) $a_{f}=a_{s}$, no strain, (b) $a_{f}>a_{s}$, in-plane compressive strain, (c) $a_{f}<a_{s}$, in-plane tensile strain.

As film thickness increases, the strain energy increases as well. At a critical thickness, the strain energy becomes too large to preserve the coherent epitaxial film growth, and it is energetically favorable for misfit dislocations to form at the interface between the film and the substrate to relieve the strain in the film, driving the lattice of the film closer to its bulk structure above the interface ${ }^{89}$ The relaxation of the film material through the formation of dislocations may have strong influence on the film morphology and physical properties. 


\section{Chapter 3 Experiments}

\subsection{Thin Film Growth}

\subsubsection{Pulsed Electron-Beam Deposition Setup}

All the thin films in this dissertation work are grown using a commercially available pulsed electron-beam deposition (PED) system by Neocera, Inc. As shown in Fig. 3-1, this system is equipped with two electron guns, allowing combinatorial deposition of two different target materials. The electron guns are capable of producing electron pulses with energies between 8 and $20 \mathrm{keV}$ at pulse frequencies up to $15 \mathrm{~Hz}$.

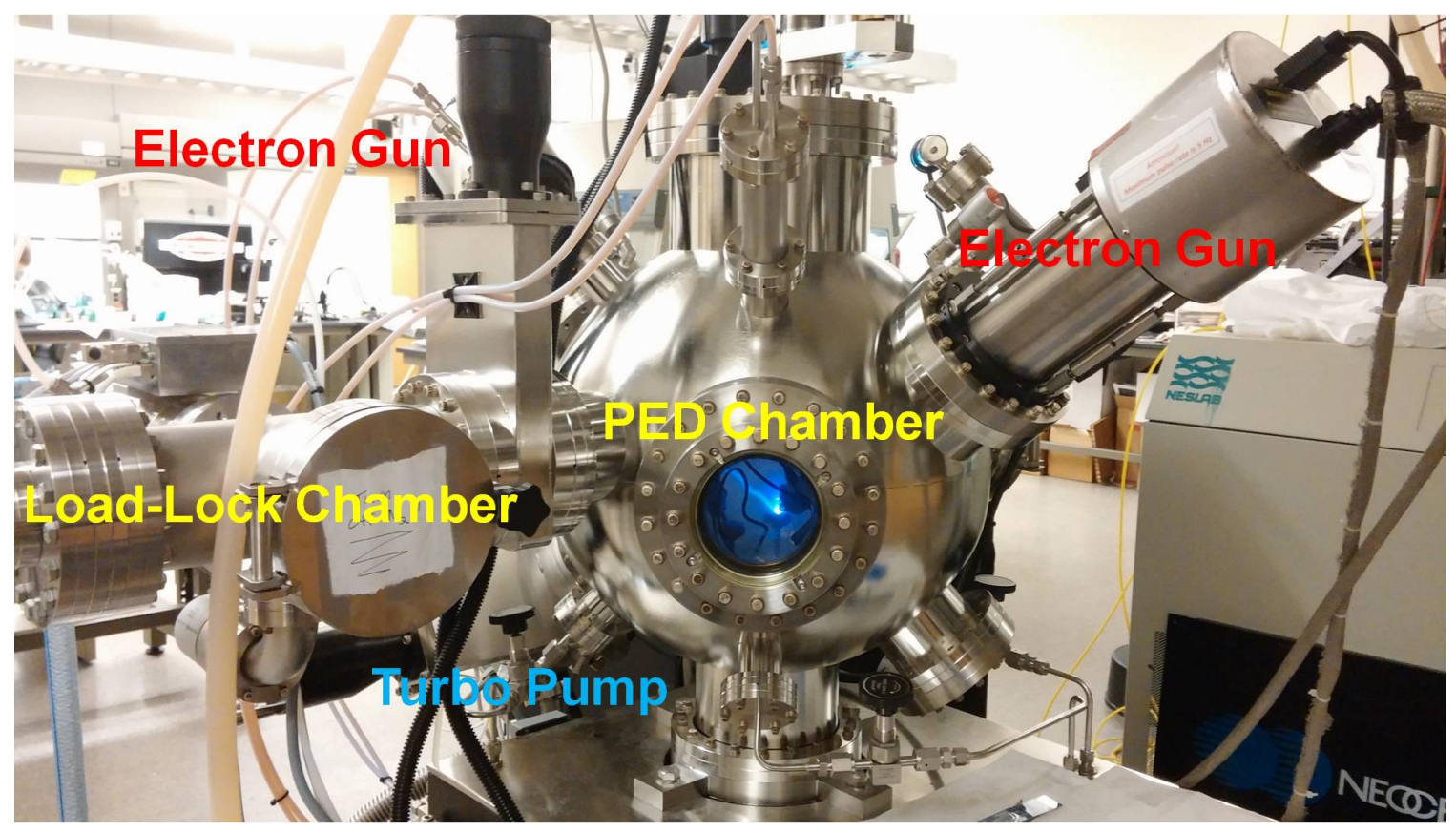

Figure 3-1 Photograph of the PED system (Neocera, Inc.). 
The base pressure of the PED chamber is $\sim 5 \times 10^{-8}$ Torr. The ultrahigh vacuum is maintained by a turbomolecular pump (Pfeiffer Vacuum Inc). A separated load-lock chamber is attached, making it possible to transfer samples without breaking the vacuum in the growth chamber. Fig. 3-2 (a) provides a view inside the PED chamber. Two electron guns are mounted on the growth chamber and electron pulses from each gun are directed toward the target surface via an alumina tube that is encased by a metallic sleeve. The gap distance between the output tube tip and the target surface, typically $2-5 \mathrm{~mm}$, is one of the most critical parameters that control the intensity of ablation, and can be adjusted by the three tension screws located on the outside of each gun. As shown in Fig. 3-2 (b), under optimal conditions for ablation, the "hot spot" on the target surface appears as a bright area of $\sim 2 \mathrm{~mm}$ in size, and is pulse-to-pulse stable in position and intensity. The plasma plume extends from the spot perpendicular to the target surface and the intensity and stability of the plume is a direct product of proper PED settings.

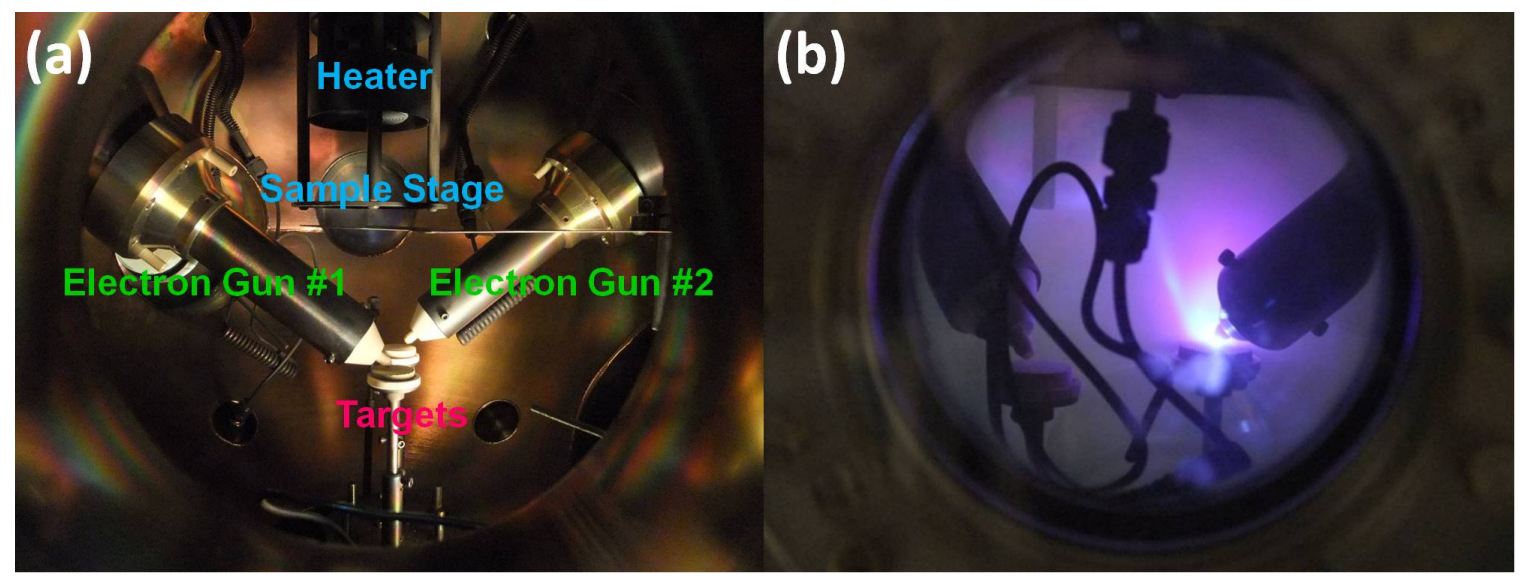

Figure 3-2 View inside the PED chamber showing (a) the relative positions of the electron guns, targets, the sample stage and heater, (b) the plasma plume produced at the surface of $\mathrm{Sr}_{2} \mathrm{~V}_{2} \mathrm{O}_{7}$ target during the deposition. 
The ceramic targets used in this dissertation work were sintered by hot-press technique (ACI Alloys, Inc.), including a $\mathrm{CaVO}_{3}$ target, a $\mathrm{Sr}_{2} \mathrm{~V}_{2} \mathrm{O}_{7}$ target and a $\mathrm{SrTiO}_{3}$ target. The targets are 1 inch in diameter and $1 / 4$ inch thick placed in alumina target holders on the target stages, where the distance between the substrate and target can be set by adjusting the height of the target stage on the carousel. To ensure the uniform ablation on the target surface for consistent plume ejection trajectory, the carousel rasters the target about its center between two angles and each target stage itself rotates. In this work, the targets were rastered between $10^{\circ}$ at a rate of $10^{\circ} / \mathrm{sec}$ and rotated about its own axis at a rate of $34^{\circ} / \mathrm{sec}$.

For the thin film deposition, the substrate is mounted on a sample plate using silver paint, and the sample plate is then transferred onto a sample stage in the growth chamber. A resistive heater is lowered onto the back of the sample plate for radiative heating up to $900{ }^{\circ} \mathrm{C}$. The presence of a small gap between the sample plate and heater allows free rotation of the sample stage to ensure the uniform deposition, however, it also leads to a temperature gradient between the heater and the surface of the sample plate. A temperature calibration has been done using a thermocouple attached to the sample plate and the data is shown in Fig. 3-3. In this work, the sample stage was rotated at $120 \% \mathrm{sec}$, and the heating/cooling rate was set at $15^{\circ} \mathrm{C} / \mathrm{min}$. Upon reaching the desired temperature, the background gas is flowed into the chamber at a rate to produce a desired processing pressure in the range of 5-20 mTorr, then the deposition process starts. The motion of the target carousel and sample stage, the background gas flow rate, along with the electron gun parameters (voltage, frequency and number of pulses) are fully software controlled. 
A manually controlled shutter can be kept in front of the substrate for pre-ablation until the conditions are properly set. The optimal deposition conditions for each material in this dissertation work will be presented in more detail in the following chapters.

Temperature Calibration

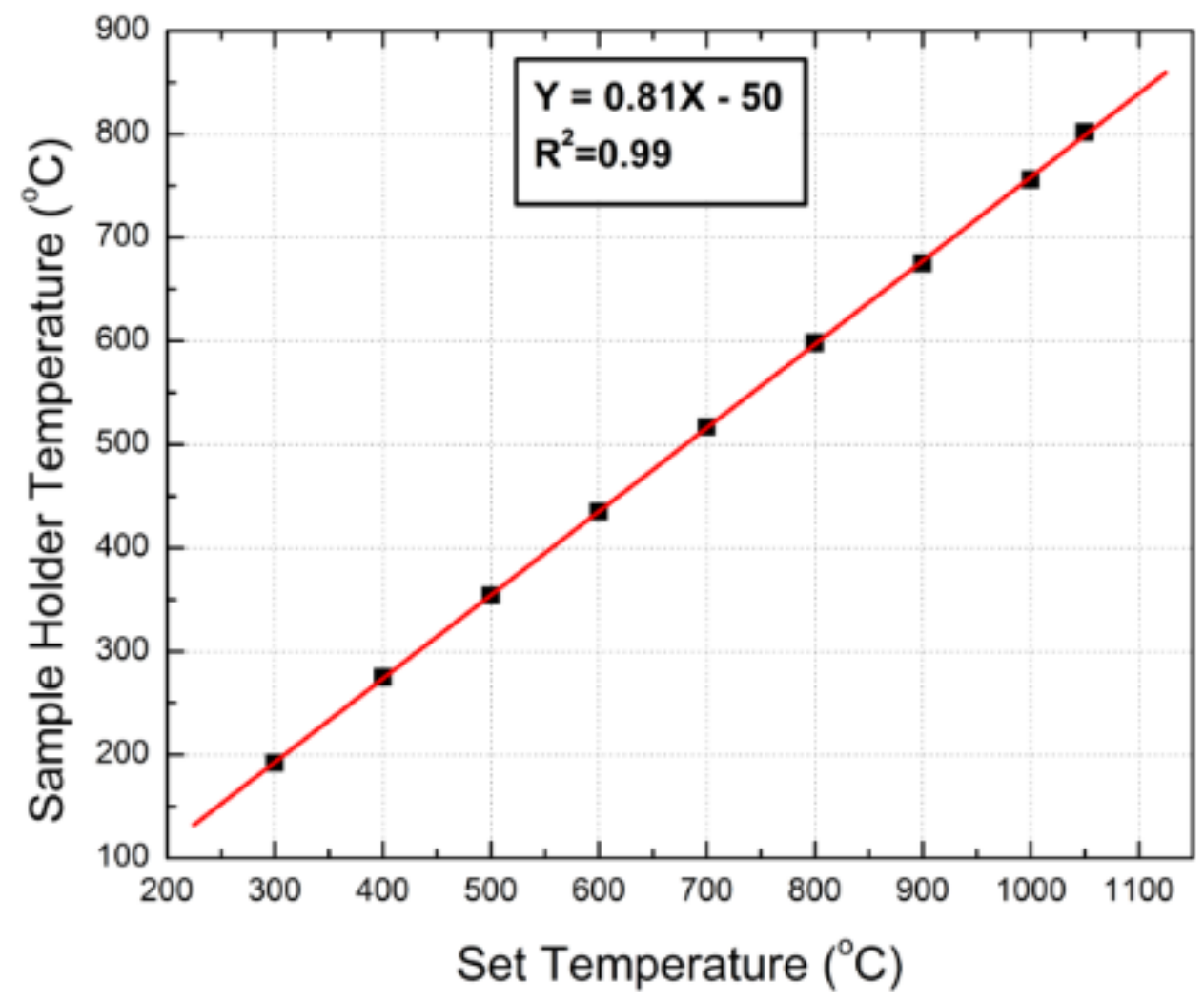

Figure 3-3 Calibration data showing the sample plate temperature as a function of the set temperature. (Adapted from Refs. 90 and 91)

\subsubsection{Substrate Preparations}

Substrates used in this study include (001)-oriented $\mathrm{SrTiO}_{3}(\mathrm{STO}$, cubic, $a=$ $3.905 \AA),\left(\mathrm{LaAlO}_{3}\right)_{0.3}\left(\mathrm{Sr}_{2} \mathrm{AlTaO}_{6}\right)_{0.7}(\mathrm{LSAT}$, cubic, $a=3.868 \AA)$, and $\mathrm{LaAlO}_{3}(\mathrm{LAO}$, pseudocubic, $a=3.79 \AA$ ). All have small lattice mismatches with the perovskite 
vanadates. The as-received substrates (Crystec, GMBH) were cleaned by a standardized procedure unless a specific surface termination is required. The cleaning procedure included sonication in acetone and isopropanol for 5 minutes each. Then the substrates were blown dry with the ultra high purity $\mathrm{N}_{2}$ gas and mounted onto the sample plate with silver paint and baked on a hotplate at $\sim 100{ }^{\circ} \mathrm{C}$ for 5 minutes. After baking, the sample plate was immediately loaded into the chamber.

In the case that single termination surfaces are desired, the substrates were treated with a well-established etching and annealing process. To prepare $\mathrm{TiO}_{2}$-terminated surfaces on STO substrates, the substrates were treated using a buffered oxide etch solution (BOE, $\mathrm{NH}_{4} \mathrm{~F}$ buffered $\mathrm{HF}$ ), followed by annealing at a high temperature. ${ }^{92}$ The steps are as follows: the STO substrates were initially ultrasonic cleaned for 5 minutes each in (1) acetone, (2) isopropanol and (3) deionized water. The substrates were then dipped in BOE solution for 30 seconds and ultrasonically cleaned in deionized water for 5 minutes, and isopropanol for 2 minutes. The substrates were then blown dry with the ultra high purity $\mathrm{N}_{2}$ gas and annealed in an open air furnace for 1 hour at $1000^{\circ} \mathrm{C}$ with a heating/cooling rate of $10^{\circ} \mathrm{C} / \mathrm{min}$. Fig. 3-4 is an AFM image of a representative STO substrate after the treatment; the topography shows highly uniform atomically smooth $\mathrm{TiO}_{2}$-terminated terraces with step heights of one unit cell $(\sim 0.4 \mathrm{~nm})$ and terrace widths of $\sim 100 \mathrm{~nm}$. 

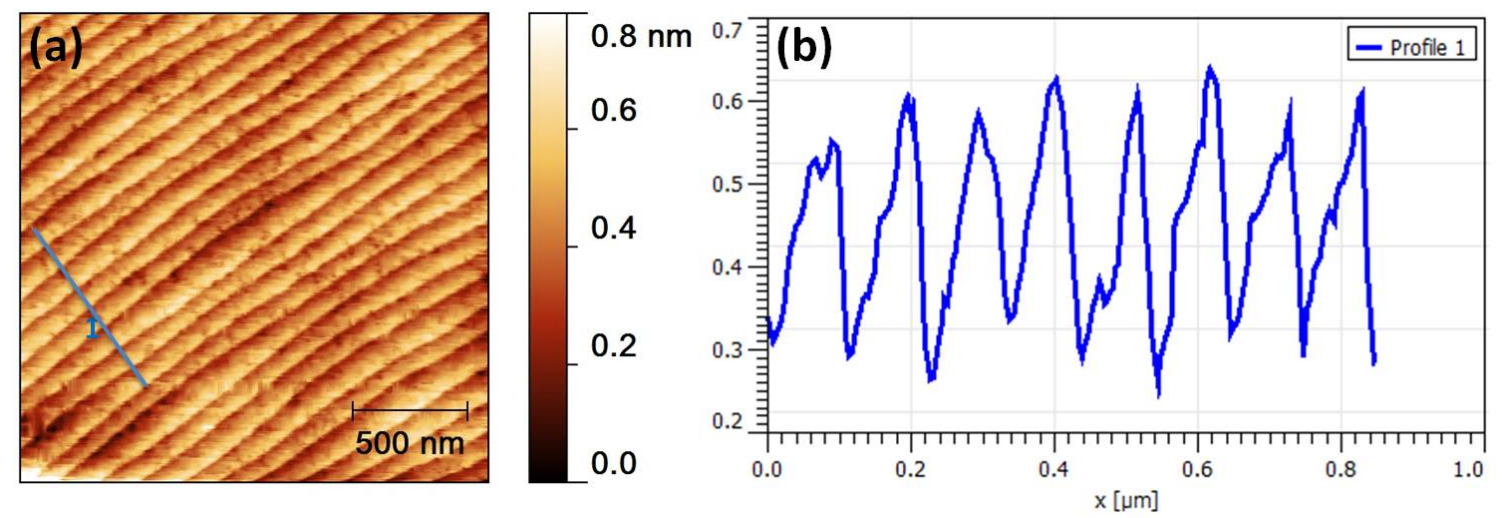

Figure 3-4 (a) AFM topography of a representative $\mathrm{SrTiO}_{3}$ (STO) substrate after the treatment. (b) Line profile along the line indicated in (a).

Single termination surfaces can be obtained on LAO substrates with a similar process in which the substrates are dipped in concentrated $\mathrm{HCl}$ for 5 minutes and annealed in air for 1 hour at $800{ }^{\circ} \mathrm{C}$. Fig. 3-5 shows an atomically flat step-and-terrace surface with step heights of one unit cell and terrace widths of $\sim 70 \mathrm{~nm}$ on a representative LAO substrate after the treatment. It has been studied that the termination layer mainly consists of $\mathrm{AlO}_{2}$, with less than $10 \%$ of $\mathrm{LaO}$ on the surface. ${ }^{93}$
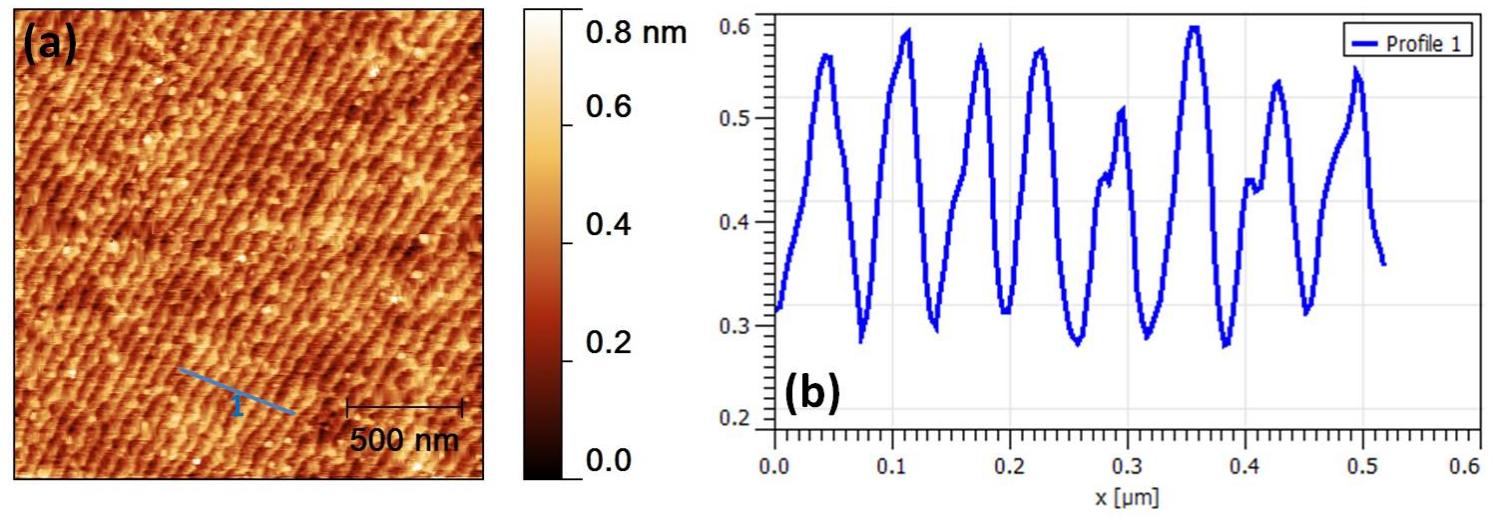

Figure 3-5 (a) AFM topography of a representative $\mathrm{LaAlO}_{3}$ (LAO) substrate after the treatment. (b) Line profile along the line indicated in (a). 


\subsection{Thin Film Characterization}

\subsubsection{Structural Characterization}

\section{X-Ray Diffraction (XRD)}

X-ray diffraction (XRD) techniques were employed in this study for the determination of structural phase and order of crystallinity of the thin film samples grown by PED. The measurements were performed using a Rigaku Smartlab diffractometer with $\mathrm{Cu} \mathrm{K} \alpha$ radiation $(\lambda=1.54 \AA)$. The $\mathrm{x}$-ray tube was operated at an accelerating voltage of $40 \mathrm{kV}$ and a beam current of $44 \mathrm{~mA}$. The system was configured for parallel beam optics, and a Ge (220) $\times 2$ mirror monochromator was used to produce a highly parallel and monochromatic x-ray beam. Samples were mounted on a 4-circle goniometer allowing for both out-of-plane and in-plane scans of the thin film samples. As shown in Fig. 3-6, the diffractometer is composed of four axes: the $\omega$ axis (sample rotation axis), the $\varphi$ axis (in-plane rotation axis), the $\psi$ axis (tilting axis) and the $2 \theta$ axis (scanning the detector).

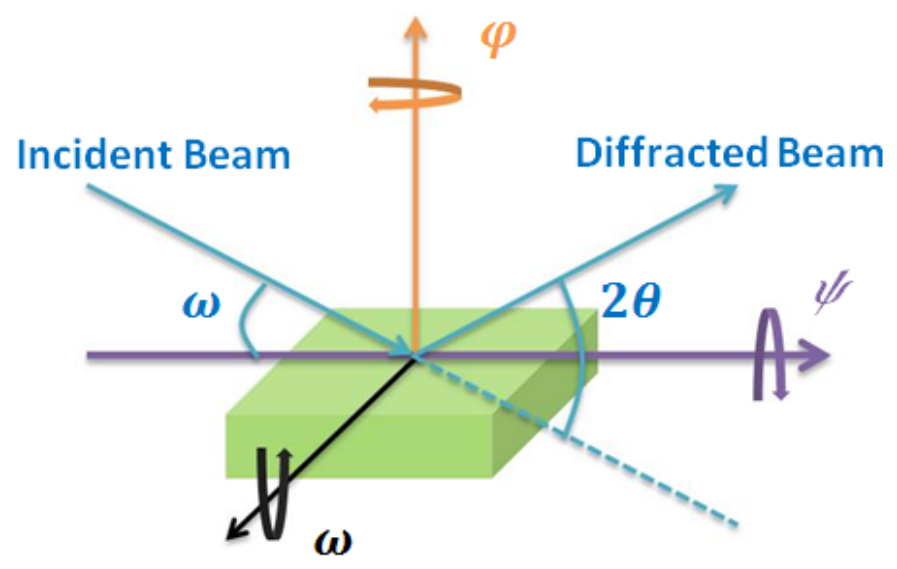

Figure 3-6 Schematic of the angles $(2 \theta, \omega, \varphi$ and $\psi)$ with respect to the sample plane for a 4-circle goniometer. 
$\mathrm{XRD}$ is a scattering technique, allowing us to learn about the crystal lattice structure of a sample by investigating its reciprocal lattice. According to Bragg's law, as illustrated in Fig. 3-7 (a), when the phases of the waves scattered from atoms in parallel planes differ by an integer multiple number of the wavelength, these waves will add constructively and result in a peak in the diffraction pattern. Bragg's law is stated as follows:

$$
n \lambda=2 d_{h k l} \sin \theta
$$

where $n$ is an integer known as the order of the diffraction, $\lambda$ is the x-ray wavelength, $d_{h k l}$ is the separation of lattice planes defined by the Miller indices $h k l$, and $\theta$ is the Bragg angle at which a peak of diffraction intensity occurs. ${ }^{94}$ Bragg's law can be formulated in reciprocal space as shown in Fig. 3-7 (b) where $\boldsymbol{k}_{\boldsymbol{0}}$ and $\boldsymbol{k}$ are the wave vectors of the incident and diffracted beam. Constructive diffraction occurs in the case:

$$
k-k_{0}=d_{h k l}^{*}
$$

where $\left|\boldsymbol{k}_{\mathbf{0}}\right|=|\boldsymbol{k}|=\frac{2 \pi}{\lambda}$, and $\boldsymbol{d}_{\boldsymbol{h} \boldsymbol{k} l}^{*}$ is a reciprocal lattice vector for the $(h k l)$ planes. ${ }^{95}$ 

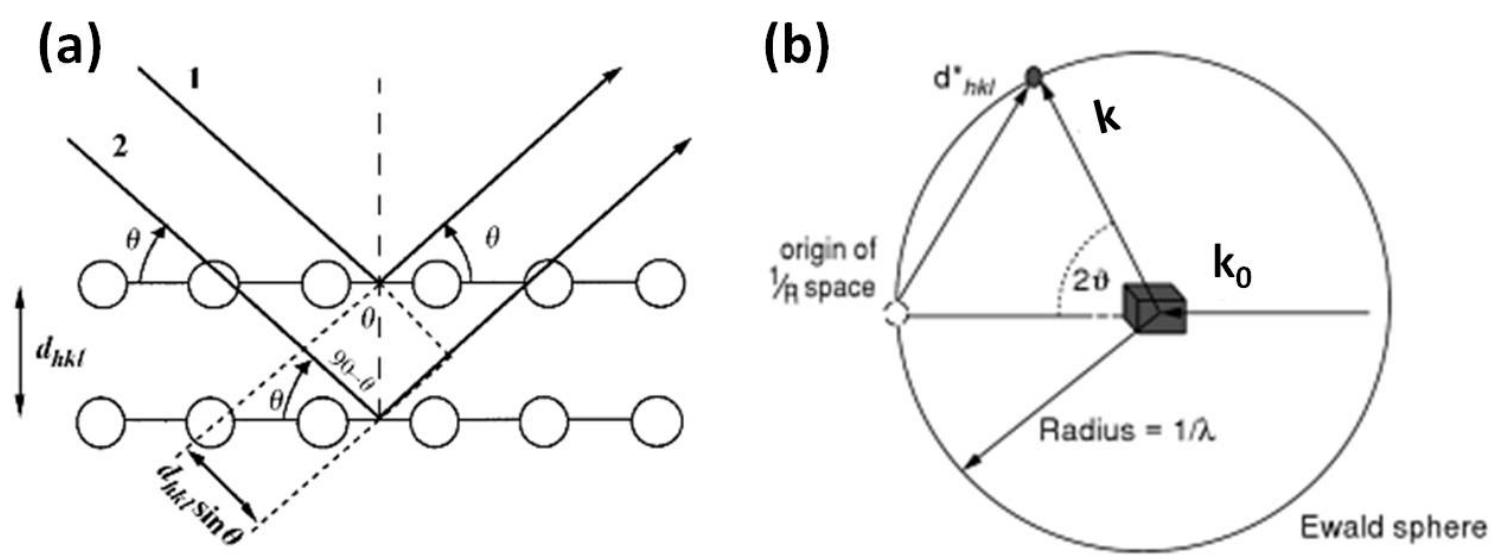

Figure 3-7 Schematic of the formulation of Bragg's law in (a) real space and (b) reciprocal space. (Adapted from Refs. 94 and 95.)

In this work, the following types of XRD techniques were commonly employed: (1) $2 \theta-\omega$ scans with $\omega=2 \theta / 2$, (2) $\omega$ scans at a particular $2 \theta$, and (3) two-dimensional off-orthogonal reciprocal space mapping by performing a set of $2 \theta-\omega$ scans with changing $\omega$. All of these scans were set to fixed $\varphi$ and $\psi$.

$2 \theta-\omega$ scans were used to determine the phase purity and preferential orientation of the thin film samples. $\omega$ represents the angle between the x-ray source and the sample plane, and $2 \theta$ is the angle between the viewing direction of the detector (or the diffracted beam) and the incident beam. These two angles were varied simultaneously with $\omega=$ $2 \theta / 2$. For a wide range $2 \theta-\omega$ scan, the angles were varied with $2 \theta$ ranging from $20^{\circ}$ to $60^{\circ}$ with step of $0.01^{\circ}$. It can be easily demonstrated that the $2 \theta-\omega$ scan is a one-dimensional scan that only scans one direction in reciprocal space. In the case of an epitaxial thin film grown on a single crystal substrate with a certain orientation, the diffraction pattern will only show the peaks produced by the film and substrate lattice planes that are parallel to 
the substrate surface such that the out-of-plane lattice parameters can be determined by using Bragg's law.

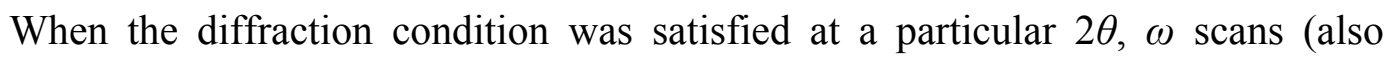
known as rocking curves) were performed in order to study the crystalline quality of the thin film samples. The detector was set to the corresponding $2 \theta$, and $\omega$ was varied in the range of $\left[\frac{2 \theta}{2}-\Delta, \frac{2 \theta}{2}+\Delta\right]$ with $\Delta=2.5^{\circ}$. As discussed above, the preferential orientation of a thin film can be determined by a symmetrical $2 \theta-\omega$ scan. The diffraction peak of a perfect epitaxial thin film would have a width of zero, but in reality, the individual crystallites in the film may slightly deviate from the preferential orientation resulting in a peak with a finite width. Rocking-curve measurements can be used to obtain the degree of the preferential orientation of a thin film. A narrow full width at half maximum (FWHM) for a film peak, which is comparable to the substrate, is a further indication of a high quality epitaxial film.

Another important technique is the reciprocal space mapping (RSM), which can be used to characterize the in-plane lattice structure and to obtain information about epitaxy, strain, and relaxation in a thin film sample. RSM is performed under an asymmetric diffraction condition, as shown in Fig. 3-8. By investigating the diffraction pattern from the lattice planes that are not parallel with the sample plane, one can obtain the reciprocal lattice vectors with non-zero in-plane components, making it possible to determine the in-plane lattice parameters. An offset angle $\delta$ is defined as the angle between the direction normal to the sample plane and the direction normal to the lattice 
planes of interest. The offset is expressed as: $\delta=\frac{2 \theta}{2}-\omega$. The value $\boldsymbol{Q}$ is defined as a scattering vector: $\boldsymbol{Q}=\boldsymbol{k}-\boldsymbol{k}_{\mathbf{0}}$, where $\boldsymbol{k}_{\mathbf{0}}$ and $\boldsymbol{k}$ are the wave vectors of the incident and diffracted beam. Diffraction occurs when $\boldsymbol{Q}$ is equivalent to a reciprocal lattice vector. $\boldsymbol{Q}$ can be written as a sum of the components parallel and perpendicular to the sample plane: $\boldsymbol{Q}=\boldsymbol{Q}_{\boldsymbol{x}}+\boldsymbol{Q}_{\boldsymbol{z}}$, as illustrated in Fig. 3-8, the magnitudes of the components are related to the angles of $2 \theta$ and $\omega$ :

$$
\begin{gathered}
\delta=\tan ^{-1}\left(\frac{Q_{x}}{Q_{z}}\right)=\frac{2 \theta}{2}-\omega \\
Q=\sqrt{Q_{x}^{2}+Q_{z}^{2}}=2 k \sin \theta=\frac{4 \pi}{\lambda} \sin \theta
\end{gathered}
$$

Therefore, when the diffraction condition is satisfied, both in-plane and out-of-plane reciprocal lattice components can be determined using the formulas below ${ }^{96}$ :

$$
Q_{x}=\frac{2 \pi}{\lambda}(\cos \omega-\cos (2 \theta-\omega)), Q_{z}=\frac{2 \pi}{\lambda}(\sin \omega+\sin (2 \theta-\omega))
$$

RSM is performed by taking a set of $2 \theta-\omega$ scans with changing $\omega$. As discussed before, a $2 \theta-\omega$ scan is a one-dimensional scan that only changes the length of the scattering vector $\boldsymbol{Q}$ but not the direction; on the other hand, the $\omega$ scan only changes its direction but not the length. By combining these two scans, a two-dimensional map of the spanned reciprocal space can be obtained. In this study, the $\{103\}$ lattice planes were chosen for RSM measurements, and the offset angle was calculated: $\delta=18.435^{\circ}$. The $2 \theta$ angle can be determined from the lattice parameters of the single crystal substrates, but for an asymmetric scan, the $\omega$ angle should be set at $\omega=\frac{2 \theta}{2}-\delta$. The range of the scans should 
cover both the substrate peak and the film peaks. Generally, $\omega$ was varied in the range of $\pm 2^{\circ}$ around the substrate peak with step of $0.02^{\circ}$, and $2 \theta$ - $\omega$ scans were performed at each $\omega$ angle. The collected data was in the goniometer coordinates $(2 \theta, \omega)$, and can be converted to reciprocal space coordinates $\left(Q_{x}, Q_{z}\right)$ by using the Rigaku 3D Explore software.

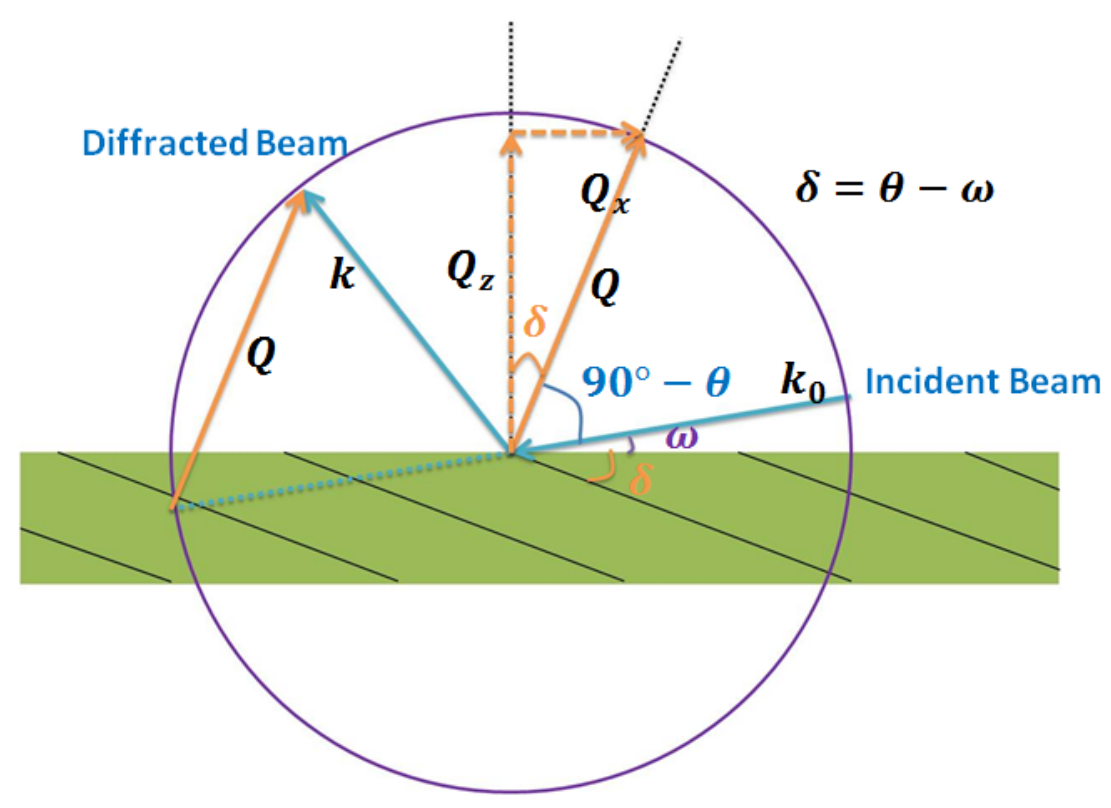

Figure 3-8 Geometry of reciprocal space mapping.

\section{X-Ray Reflectivity (XRR)}

Film thickness was determined by an $\mathrm{x}$-ray reflectivity (XRR) technique. $2 \theta-\omega$ scans with $2 \theta$ varied from $0^{\circ}$ to $6^{\circ}$ were performed to measure the reflectivity profiles using the Rigaku Smartlab XRR software package. In this method, an x-ray beam irradiates a thin film sample at grazing angles to avoid the dominance of Bragg diffraction. The interference of x-ray beams reflected by the different interfaces creates a 
reflectometry pattern that provides important information about the film thickness. ${ }^{97}$ For an incident angle below a critical angle, the incident x-ray beam is totally reflected by the sample surface. The critical angle is related to the refractive index of a material, which depends on the material's electron density. Generally, the critical angles for most materials are less than $1^{\circ}$. As the incident angle gradually increases beyond the critical angle, the incident $\mathrm{x}$-ray beam penetrates into the material and the reflection from the different interfaces of the sample gives rise to interference fringes also known as Kiessig fringes. The amplitude of the fringes depends on the difference in electron density at the interfaces as larger difference leads to higher amplitude. Also, the reflected intensity decays more rapidly with increasing roughness at the surface and interface due to the diffuse scattering. ${ }^{98}$ The film thickness $t$ can be estimated from the periodicity of the fringes:

$$
t \approx \lambda / \Delta 2 \theta
$$

where $\lambda$ is the $\mathrm{x}$-ray wavelength, and $\Delta 2 \theta$ is the $2 \theta$ angular separation between consecutive interference peaks. ${ }^{99}$

In this study, the reflectivity data was analyzed using the Rigaku GXRR software. The software is an effective tool for simulating the reflectivity profile for determination of film thickness, density and roughness, especially for multilayer films with more complex reflectivity profiles. Fig. 3-9 shows the experimental XRR profile of a $\mathrm{SrVO}_{3}$ thin film grown on LSAT substrate and a fit of the data. The film was characterized to be $27.5 \mathrm{~nm}$ thick, with the density of $5.296 \mathrm{~g} / \mathrm{cm}^{3}$ and roughness of $0.88 \mathrm{~nm}$. 


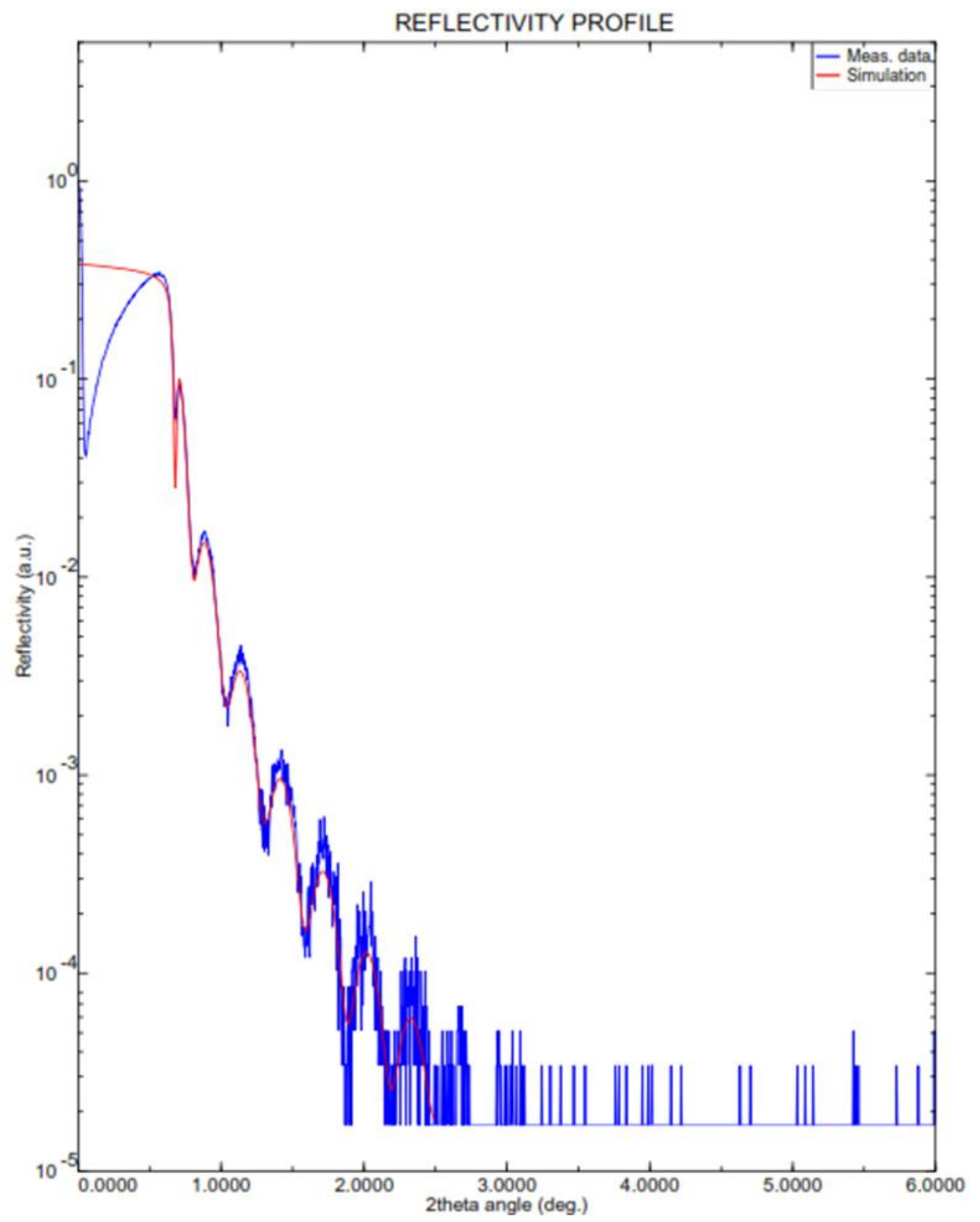

Figure 3-9 A representative XRR profile of a $\mathrm{SrVO}_{3}$ thin film grown on LSAT substrate (blue color), along with a fitted curve (red color). 


\section{X-Ray Absorption Spectroscopy (XAS)}

The electronic structures of a few films in this study were examined by $\mathrm{x}$-ray absorption spectroscopy (XAS). XAS measures the absorption of x-rays as a function of photon energy. When an x-ray photon of sufficient energy is absorbed, a core-level electron is excited to a higher energy unoccupied state above the Fermi level, leaving behind a highly excited core-hole state. ${ }^{100}$ The core-hole that is created will be refilled either by a non-radiative process, emitting an Auger electron, or a radiative process, emitting x-ray fluorescence. The intensities of these decay channels are proportional to the number of core-holes created by the absorption process. Therefore, the x-ray absorption process can be measured in either total electron yield (TEY) or total fluorescent yield (TFY) mode. In the TEY mode, Auger electrons are scattered inelastically as they escape from the sample, leading to a secondary electron cascade. The TEY signal is dominated by the secondary electrons, since their amount is orders of magnitude larger than the amount of the original Auger electrons. The mean free path of these secondary electrons is in the range of 50-100 $\AA$, limiting the TEY probing depth to a surface layer of about the same thickness. On the other hand, the TFY mode is quite bulk sensitive, and the TFY probing depth is $\sim 2000 \AA$ since photons have a greater escape depth than electrons. ${ }^{101}$

In this study, XAS measurements were performed at beamline 8.0.1 of the Advanced Light Source at Lawrence Berkeley Laboratory by our collaborator Jude Laverock at Boston University. Measurements were made in both TEY and TFY modes 
with an energy resolution set to $\leq 0.3 \mathrm{eV}$ at full width half maximum, and the photon energy was calibrated using $\mathrm{TiO}_{2}$ reference spectra of the Ti $L$-edge and $\mathrm{O} K$-edge.

\section{Atomic Force Microscopy (AFM)}

The film surface morphology was analyzed by atomic force microscopy (AFM) (Cypher, Asylum Research Inc.) operated in tapping mode. A schematic of the AFM instrument is shown in Fig. 3-10.

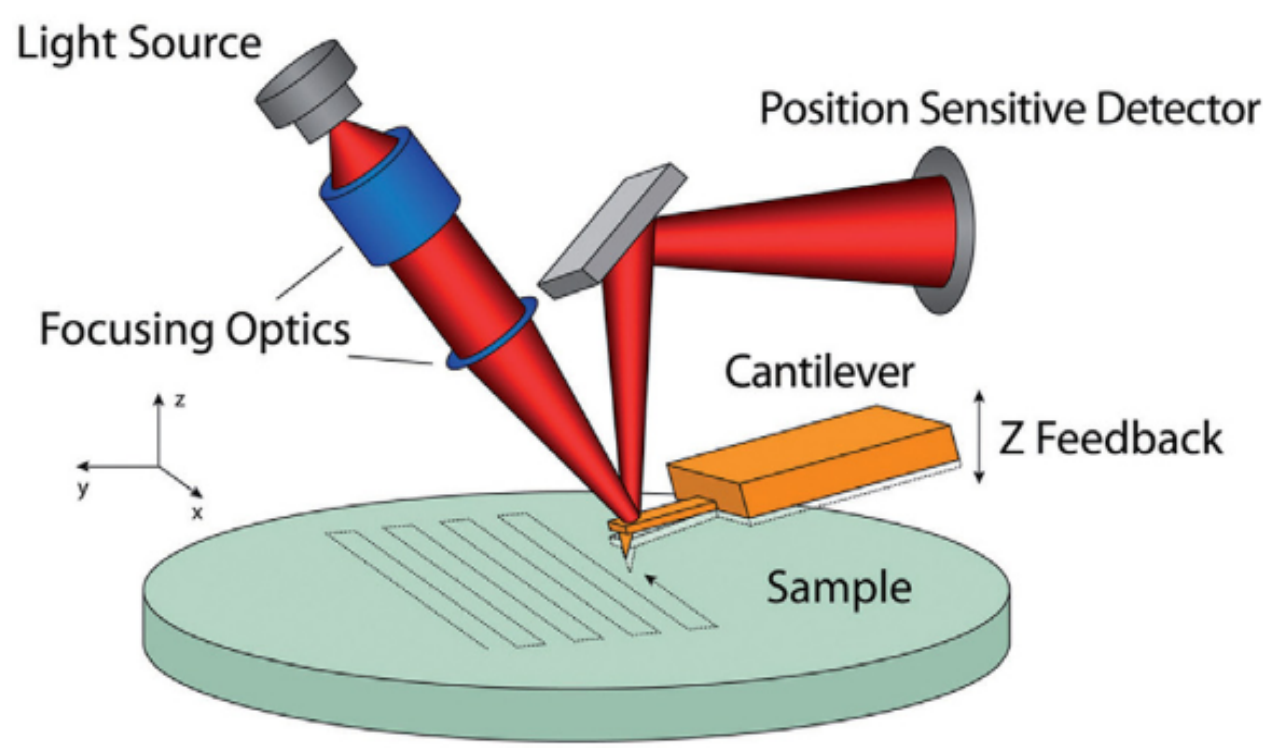

Figure 3-10 Schematic of AFM instrument. (Adapted from Ref. 102)

AFM provides a three-dimensional surface topography map of a sample in the nanoscale by probing the surface with a sharp tip of $\sim 10 \mathrm{~nm}$ in radius attached to the end of a cantilever. As the cantilever raster-scans the sample surface, the small force between the tip and surface bends the cantilever and its deflection is optically detected. A laser beam is reflected off the back of the cantilever and detected by a four quadrant 
photodiode. In tapping mode, the cantilever is oscillated near its resonance frequency. As the tip comes close the sample surface, the force between the tip and surface causes a change in the oscillation amplitude. An electronic feedback loop adjusts the height of the cantilever above the sample surface using a piezoelectric actuator to ensure the oscillation amplitude remains constant. Therefore, by scanning the tip over the entire surface area of interest, the surface topography is obtained. ${ }^{102}$

\subsubsection{Transport Characterization}

Transport properties of the thin film samples were characterized using a Physical Property Measurement System (PPMS) (Quantum Design Inc.) in the temperature range of 2-300 K. The van der Pauw technique was used to perform resistivity measurements. In this method, two characteristic resistances $R_{A}$ and $R_{B}$ are measured using the configuration shown in Fig. 3-11 (a), and the sheet resistance $R_{S}$ can be determined by numerically solving the equation: ${ }^{103}$

$$
\exp \left(-\pi R_{A} / R_{S}\right)+\exp \left(-\pi R_{B} / R_{S}\right)=1
$$

The bulk electrical resistivity can be calculated by $\rho=R_{S} d$, where $d$ is the thickness of the film.

In the resistivity measurements, cold-welded indium contacts were made at the

four corners of the $5 \times 5 \mathrm{~mm}^{2}$ square-shaped samples, and the samples were bonded to a PPMS resistivity puck using Au wires as shown in Fig. 3-11 (b). An excitation current of $10 \mu \mathrm{A}$ was generally applied. Using the same van der Pauw configuration, 
magnetoresistance (MR) (defined as $[R(B)-R(0)] / R(0)$ ) was measured with a perpendicular magnetic field up to $7 \mathrm{~T}$.

(a)

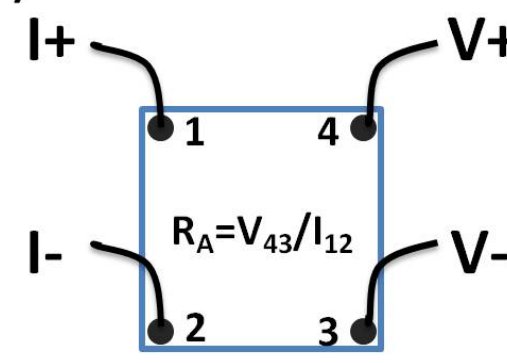

$\mathrm{V}+\mathrm{V}+$

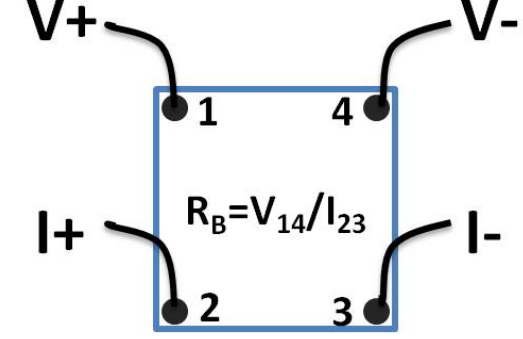

(b)

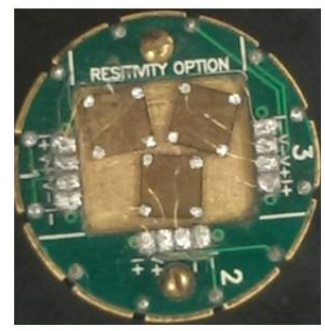

Figure 3-11 (a) Schematic of a van der Pauw configuration used in the determination of sheet resistance. (b) A representative photo of samples bonded to a PPMS sample puck for resistivity measurements.

Hall effect measurements were performed to determine the carrier concentration and mobility. The Hall effect is illustrated in Fig. 3-12 (a). When an electric current flows through the sample with a perpendicular magnetic field, the magnetic field exerts a transverse force on the moving charge carriers, resulting in a potential drop across the two sides of the sample known as Hall voltage $V_{H} \cdot{ }^{103}$ The bulk carrier concentration is given by:

$$
n=I B / e d V_{H}=B / e d R_{H}
$$

where $I$ is current, $B$ is magnetic field, $e$ is the elementary charge, $d$ is the sample thickness, and $R_{H}$ is the Hall resistance. The mobility $\mu$ can be determined by $\mu=1 /$ en $\rho$, where $\rho$ is the bulk resistivity. The sample configuration for Hall measurements is shown in Fig. 3-12 (b). Hall resistances are measured as a function of magnetic field and the sign 
of the slope indicates the carrier type with a negative (positive) slope corresponding to electrons (holes).

(a)

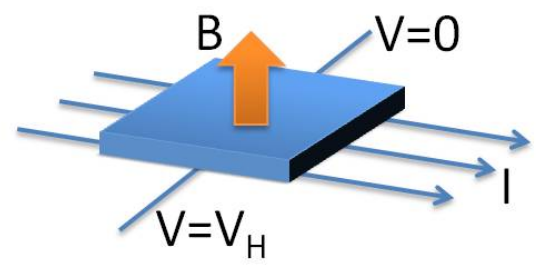

(b)

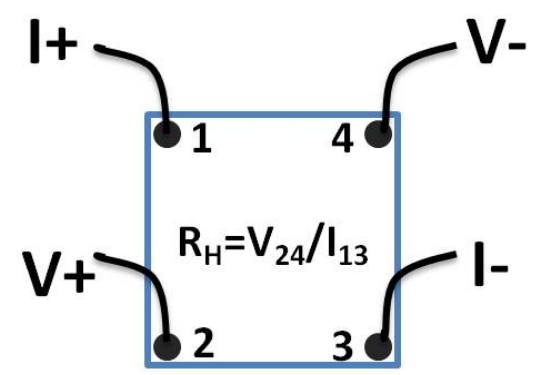

Figure 3-12 Schematic of (a) Hall effect and (b) sample configuration in the Hall effect measurement. 


\section{Chapter 4 Epitaxial Vanadate Thin Films}

\subsection{Introduction}

Perovskite vanadates $\mathrm{SrVO}_{3}(\mathrm{SVO})$ and $\mathrm{CaVO}_{3}(\mathrm{CVO})$ are intriguing correlated metals near a metal-insulator transition (MIT) ${ }^{26}$ However, due to the great challenges in the synthesis of these materials, the physical properties of the vanadate thin films have been rarely studied. This chapter describes the synthesis of epitaxial SVO and CVO thin films via a novel pulsed electron-beam deposition (PED) technique. PED is a rather complex process and the important parameters to be optimized include electron gun potential, pulse frequency, electron gun tube tip-to-target distance, target-to-substrate distance, target rotation and raster speed, background gas composition and pressure, and deposition temperature, etc. In this chapter, I will examine and discuss microstructures and transport properties of SVO and CVO films grown under optimal conditions.

\section{2 $\mathrm{SrVO}_{3}$ Thin Films}

\subsubsection{Film Growth}

High quality epitaxial $\mathrm{SrVO}_{3}(\mathrm{SVO})$ thin films were deposited on three different substrates by PED, including (001)-oriented $\mathrm{LaAlO}_{3}(\mathrm{LAO}),\left(\mathrm{LaAlO}_{3}\right)_{0.3}\left(\mathrm{Sr}_{2} \mathrm{AlTaO}_{6}\right)_{0.7}$ (LSAT) and $\mathrm{SrTiO}_{3}$ (STO). The lattice parameters and mismatch relative to SVO are listed in Table 4-1. For all the substrates, the lattice mismatch between the substrates and SVO $(a=3.843 \AA)$ is less than $2 \%$. Both LAO and STO substrates were prepared to have single terminated step-and-terrace surfaces on an atomic scale using the methods 
described in Chapter 3. During the deposition, a $12 \mathrm{kV}$ potential in the electron gun was applied to ablate a ceramic target of $\mathrm{Sr}_{2} \mathrm{~V}_{2} \mathrm{O}_{7}$ at a rate of $5 \mathrm{~Hz}$, and the SVO thin films were deposited at a temperature of $800{ }^{\circ} \mathrm{C}$ in a $10 \mathrm{mTorr} \mathrm{Ar}$ atmosphere. The growth conditions are also listed in Table 4-2. The film thickness was determined by using x-ray reflectivity (XRR) and the deposition rate was found to be $\sim 2 \AA / \mathrm{min}$.

Table 4-1 Substrate lattice parameters and mismatch to SVO.

\begin{tabular}{cccc}
\hline Substrate & LAO & LSAT & STO \\
\hline Lattice parameter $(\AA)$ & 3.79 & 3.868 & 3.905 \\
Lattice mismatch to SVO & $+1.38 \%$ & $-0.65 \%$ & $-1.61 \%$ \\
\hline
\end{tabular}

Table 4-2 Growth conditions of SVO films.

\begin{tabular}{cccc}
\hline $\begin{array}{c}\text { Voltage } \\
(\mathbf{k V})\end{array}$ & $\begin{array}{c}\text { Pulse Rate } \\
(\mathbf{H z})\end{array}$ & $\begin{array}{c}\text { Ar Pressure } \\
(\mathbf{m T o r r})\end{array}$ & $\begin{array}{c}\text { Temperature } \\
\left({ }^{\circ} \mathbf{C}\right)\end{array}$ \\
\hline 12 & 5 & 10 & 800 \\
\hline
\end{tabular}

\subsubsection{Structure and Morphology}

AFM topography images of $6.5 \mathrm{~nm}$ SVO films grown on three different substrates are shown in Fig. 4-1. All the images showed step-terrace structures and atomically flat film surfaces with RMS roughness of $\sim 0.2 \mathrm{~nm}$. SVO films grow in a layer-by-layer mode on LAO and LSAT substrates. The film grown on STO substrate showed islands within the terrace, indicating layer-plus-island growth. The inset AFM line scans also indicated step-terrace structures with each step height close to one unit cell lattice spacing of $\sim 4 \AA$. The terrace widths were found to be $125 \mathrm{~nm}, 230 \mathrm{~nm}$ and $150 \mathrm{~nm}$ for the films grown on 
LAO, LSAT and STO substrates, respectively. The different terrace widths may result from different substrate miscut angles.
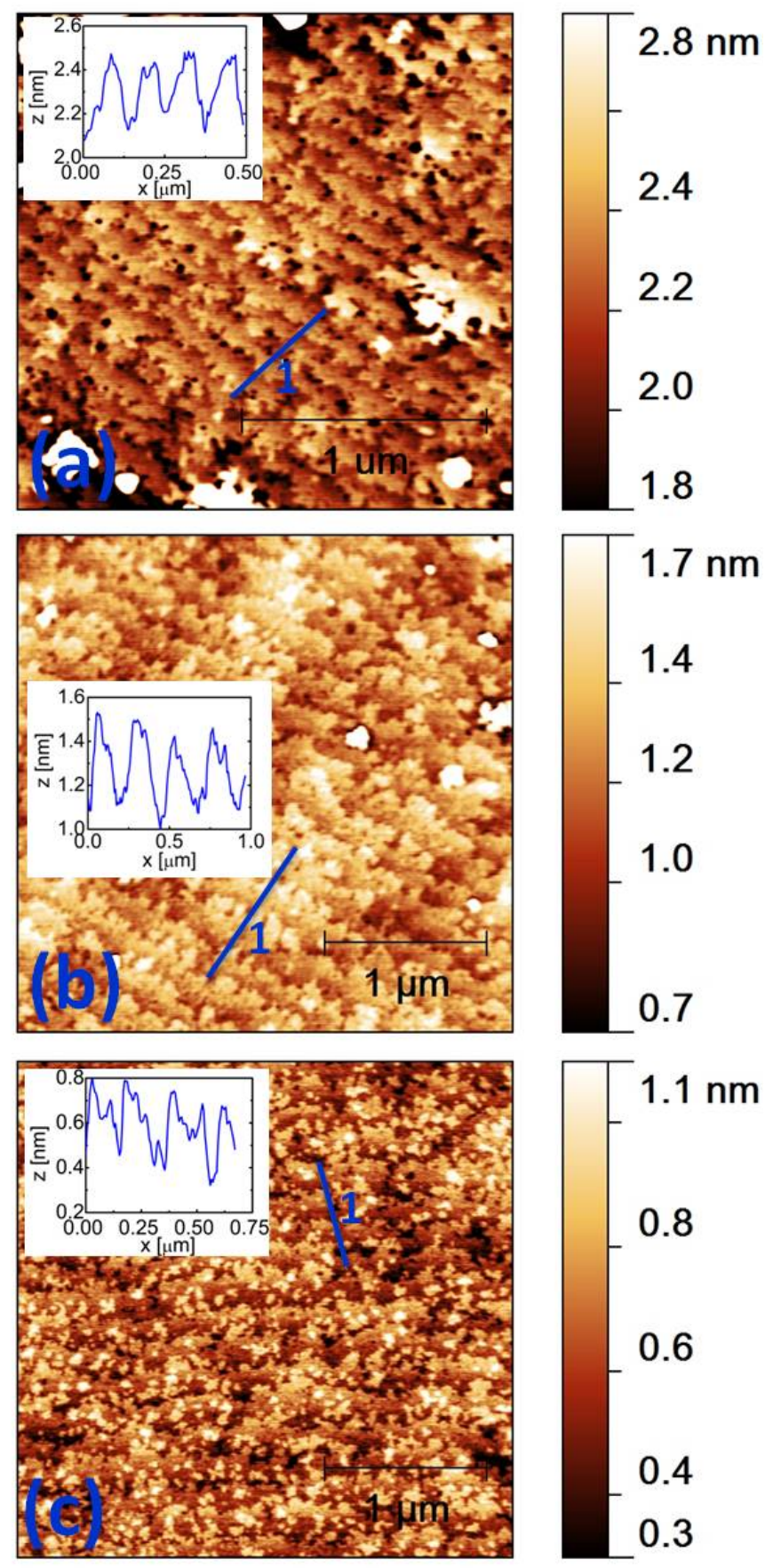

Figure 4-1 AFM topography images and (inset) line scans of $6.5 \mathrm{~nm} \mathrm{SVO}$ films grown on (a) LAO, (b) LSAT and (c) STO substrates. 
Fig. 4-2 shows out-of-plane XRD scans of $25 \mathrm{~nm}$ SVO films grown on three different substrates. The wide range scans confirmed single phase epitaxial SVO films on all three substrates. Moreover, the thickness fringes (Kiessig fringes) were observed around the (002) symmetric reflection. These thickness fringes result from the constructive interference of the x-ray beams reflected at the interface between the film and the substrate, which can thereby be used to quantify the film thickness. The periodicity of the thickness fringes $\Delta \theta$ and the film thickness $t$ are related by: $\Delta \theta=$ $\frac{\lambda}{2 t \cos \theta_{B}}$, where $\lambda$ is the x-ray wavelength and $\theta_{B}$ is the Bragg diffraction angle. ${ }^{104}$ The calculated film thicknesses agree with the results obtained from XRR measurements. The presence of these thickness fringes indicated smooth and coherent SVO films with high quality film/substrate interfaces.
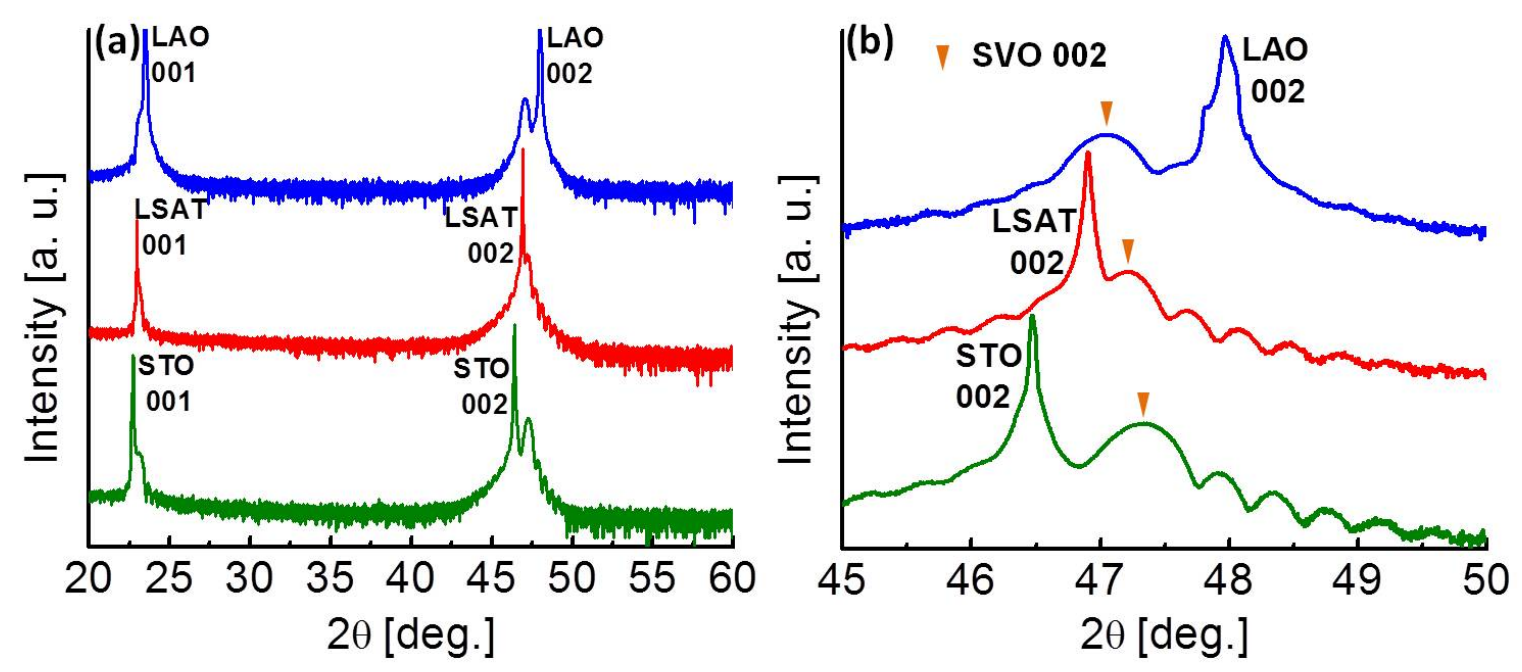

Figure 4-2 (a) Wide range XRD scans of $25 \mathrm{~nm}$ SVO films grown on three different substrates showing that no secondary phases are present. (b) XRD scans around (002) reflection showing Kiessig fringes. The triangles indicate the SVO (002) peak positions. 
The out-of-plane lattice parameters of SVO films grown on three different substrates can be easily calculated from (002) peaks of the film as shown in Fig. 4-2 (b) using Bragg's law. To determine the in-plane lattice parameters, reciprocal space mapping (RSM) measurements were performed on the same SVO films. Fig. 4-3 shows the RSM on the (103) asymmetric reflection, where the reciprocal lattice coordinate $Q_{z}$ represents the out-of-plane [001] direction while the coordinate $Q_{x}$ represents the inplane [100] direction. As shown in Fig. 4-3 (b) and (c), both the film and the substrate peaks fall at the same value of $Q_{x}$ for the SVO films grown on LSAT and STO. This indicates coherent growth of the film with the same in-plane lattice parameter as the underlying substrate. On the other hand, the RSM in Fig. 4-3 (a) indicates strain relaxation in the SVO film grown on LAO. Table 4-3 lists the out-of-plane and in-plane epitaxial strain in the SVO films grown on the three substrates determined from lattice parameters.

Table 4-3 Out-of-plane and in-plane epitaxial strain in the $25 \mathrm{~nm}$ SVO films grown on three different substrates.

\begin{tabular}{cccc}
\hline Substrate & LAO & LSAT & STO \\
\hline Out-of-plane epitaxial strain (\%) & 0.44 & 0.08 & -0.16 \\
In-plane epitaxial strain (\%) & -0.08 & 0.65 & 1.61 \\
\hline
\end{tabular}

Twinning is commonly observed in LAO substrates. Single crystal LAO undergoes a structural phase transition from a rhombohedral $(a=3.79 \AA)$ to a cubic ( $a=$ $3.81 \AA$ A) structure at $435^{\circ} \mathrm{C}$. The formation of twin microstructures in LAO results from the phase transition during cool-down after crystal growth. ${ }^{105,106}$ The twinning in LAO 
may result in poor quality film/substrate interface, leading to strain relaxation in the deposited SVO film during cool-down from the growth temperature. As a result, the intensities of the thickness fringes around the (002) symmetric reflection for the SVO film grown on LAO are much lower than the films grown on LSAT and STO (Fig. 4-2).
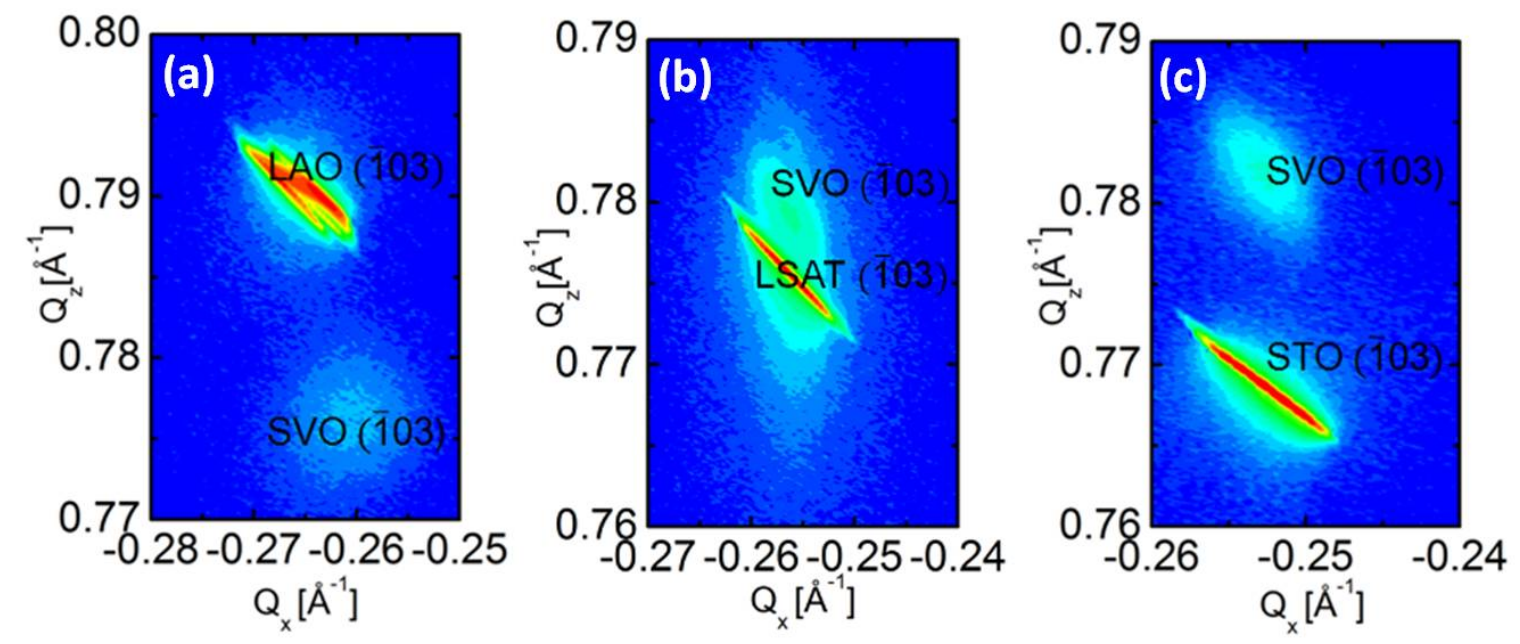

Figure 4-3 RSM on (103) reflection of $25 \mathrm{~nm}$ SVO films grown on (a) LAO, (b) LSAT and (c) STO substrates.

\subsubsection{Transport Properties}

To explore the electrical transport properties of SVO films, sheet resistances of 25 nm SVO films grown on LAO, LSAT and STO substrates were measured as a function of temperature. As shown in Fig. 4-4 (a), all the SVO films grown on the three different substrates exhibited metallic behaviors in the entire temperature range of $2-300 \mathrm{~K}$ and the sheet resistances decreased with decreasing temperature. The sheet resistances of SVO films grown on LSAT and STO were quite close, however the sheet resistance of the film grown on LAO was found to be $\sim 1.5$ times higher than that of the films grown on LSAT 
and STO. This may result from strain relaxation via the formation of dislocations in the SVO film grown on LAO.
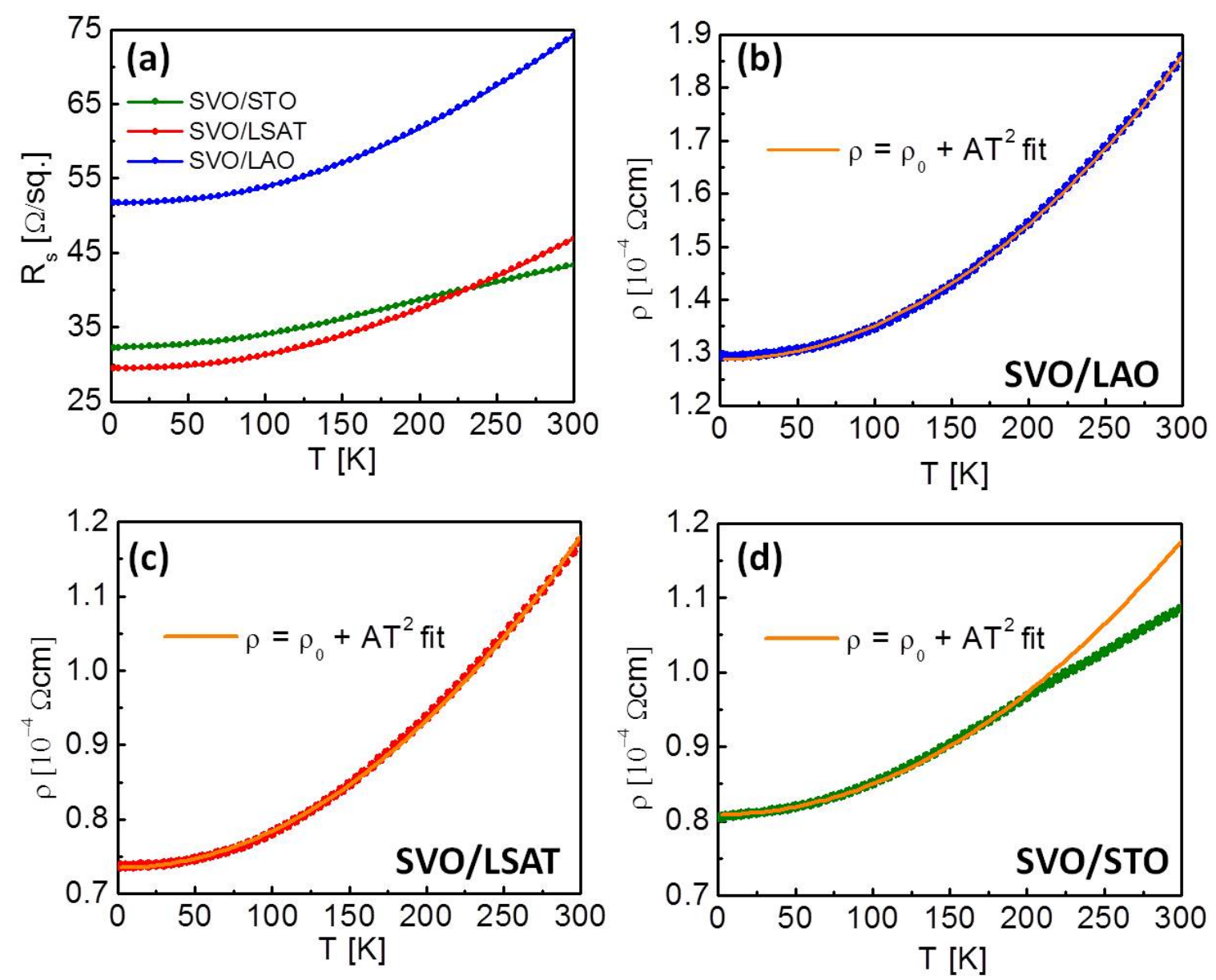

Figure 4-4 (a) Sheet resistance vs. temperature for $25 \mathrm{~nm}$ SVO films grown on three different substrates. Temperature dependence of electrical resistivity of the same SVO films grown on (b) LAO, (c) LSAT and (d) STO substrates. $\rho=\rho_{0}+A T^{2}$ fits are also shown.

The electrical resistivity $\rho$ of the film can be obtained from the sheet resistance $R_{S}$ by $\rho=R_{S} d$, where $d$ is the film thickness. Fig. 4-4 (b)-(d) show the temperature dependence of electrical resistivity of the same SVO films grown on the three different substrates. For all the SVO films, the electrical resistivity was $\sim 10^{-4} \Omega \mathrm{cm}$ at room 
temperature and the resistivity ratio $\rho(300 \mathrm{~K}) / \rho(2 \mathrm{~K})$ was in the range of $1.3 \sim 1.6$. Moreover, the electrical resistivity as a function of temperature can be expressed by a $\rho=$ $\rho_{0}+A T^{2}$ relation corresponding to a Fermi liquid system, where the residual resistivity $\rho_{0}$ is a temperature-independent value attributed to the electron-impurity scattering caused by defects, and the coefficient $A$ quantifies the electron-electron interactions in the strongly correlated system. $\rho=\rho_{0}+A T^{2}$ fits are also shown in Fig. 4-4 (b)-(d). For the SVO film grown on STO, the resistivity slightly deviated from the $T^{2}$ dependence as the temperature was raised above $200 \mathrm{~K}$. The fitting parameters of $\rho_{0}$ and $A$ are listed in Table 4-4. The values of SVO films grown on LSAT and STO are consistent with the reported values of bulk SVO. ${ }^{57}$ The higher residual resistivity $\rho_{0}$ observed in the SVO grown on LAO indicates more defects in the film.

Table 4-4 Transport data of $25 \mathrm{~nm}$ SVO films grown on the three different substrates.

\begin{tabular}{cccc}
\hline & SVO/LAO & SVO/LSAT & SVO/STO \\
\hline$\rho_{0}\left[10^{-5} \Omega \mathbf{c m}\right]$ & 12.9 & 7.35 & 8.09 \\
$\mathbf{A}\left[\mathbf{1 0}^{-10} \boldsymbol{\Omega} \mathbf{c m} / \mathbf{K}^{2}\right]$ & 6.37 & 4.95 & 4.09 \\
$\boldsymbol{\rho}(\mathbf{3 0 0} \mathbf{K}) / \boldsymbol{\rho}(\mathbf{2} \mathbf{K})$ & 1.44 & 1.59 & 1.35 \\
\hline
\end{tabular}

The Hall effect of the same $25 \mathrm{~nm}$ SVO films was investigated over the temperature range of 2-300 K, as shown in Fig. 4-5. For all the films grown on the three different substrates, the carriers were found to be electrons with a concentration of $\sim 3 \times$ $10^{22} \mathrm{~cm}^{-3}$, which is on the same order of magnitude as the predicted value of $1.8 \times 10^{22}$ $\mathrm{cm}^{-3}$ assuming a simple one-band model. The electron mobility was in the range of 1-2 $\mathrm{cm}^{2} / \mathrm{Vs}$ at room temperature, the low electron mobility is as expected from a strongly 
correlated system. ${ }^{42}$ The electron mobility slightly increased with decreasing temperature, while the carrier concentration stayed relatively constant. The temperature dependence of the electron mobility agreed with the resistivity ratio $\rho(300 \mathrm{~K}) / \rho(2 \mathrm{~K})$ listed in Table 4-4.
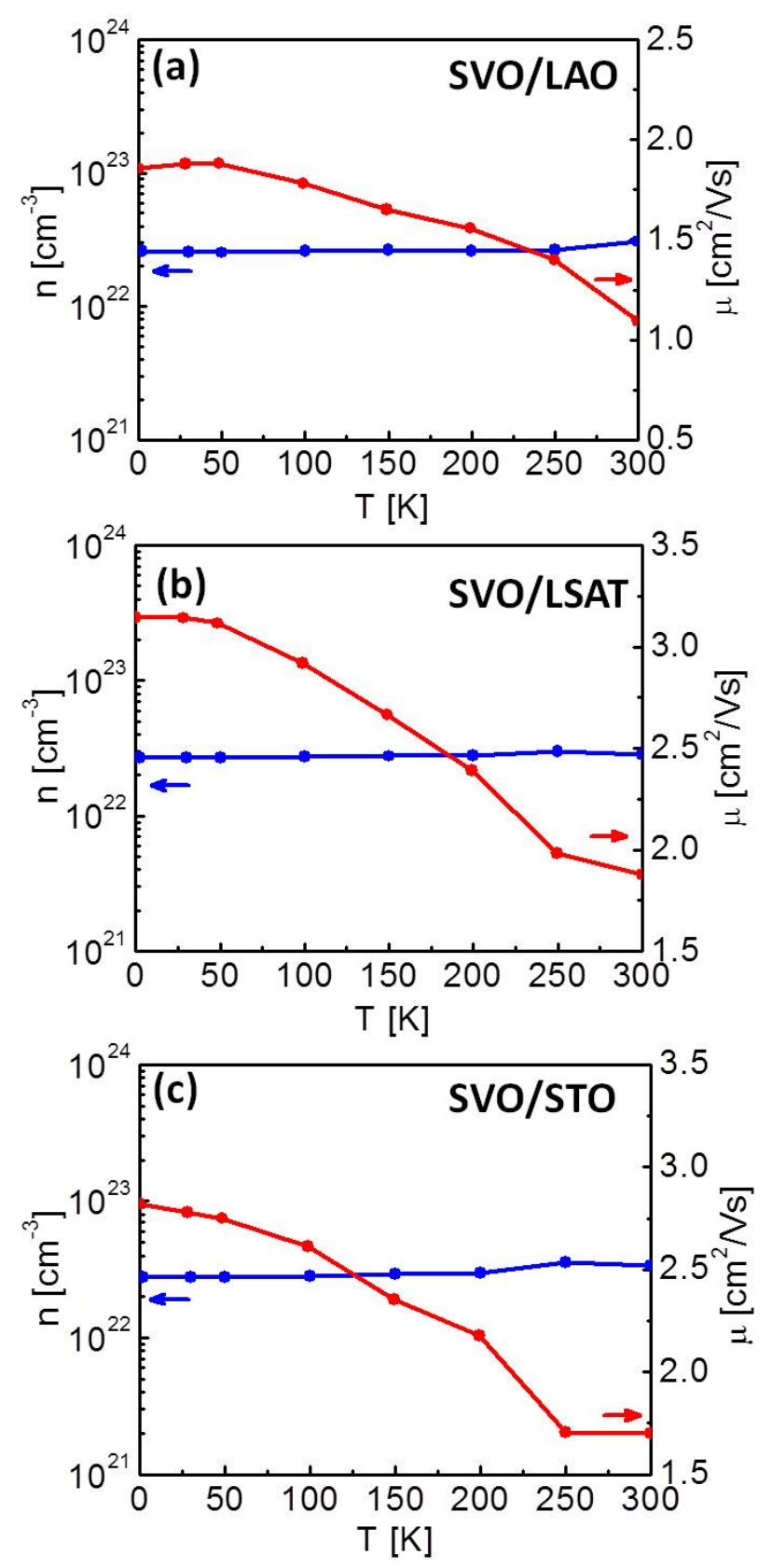

Figure 4-5 Temperature dependence of carrier density and mobility from Hall effect measurements of the same $25 \mathrm{~nm}$ SVO films grown on (a) LAO, (b) LSAT, and (c) STO substrates. 


\section{3 $\mathrm{CaVO}_{3}$ Thin Films}

\subsubsection{Film Growth}

$\mathrm{CaVO}_{3}(\mathrm{CVO})$ thin films were grown by PED on (001)-oriented $\mathrm{SrTiO}_{3}$ (STO) substrates with a stoichiometric CVO ceramic target. The STO substrates were prepared to have atomically smooth $\mathrm{TiO}_{2}$-terminated surfaces, and the lattice mismatch between the film and the substrate is $-3.6 \%$. During the deposition, the substrates were heated to $650{ }^{\circ} \mathrm{C}$ in a $7 \mathrm{mTorr}$ Ar atmosphere. A $15.4 \mathrm{kV}$ potential in the electron gun was applied to ablate the CVO target at a rate of $9 \mathrm{~Hz}$. The film growth conditions are listed in Table 4-5. Rutherford backscattering spectrometry (RBS) measurements determined that the $\mathrm{Ca} / \mathrm{V}$ ratio was $\sim 1$ in the $\mathrm{CVO}$ films. To protect the surface of $\mathrm{CVO}$, a $2.5 \mathrm{~nm} \mathrm{STO}$ capping layer was then deposited in-situ using a STO target. X-ray reflectivity (XRR) measurements were performed to determine the film thickness and the deposition rate was found to be $0.45 \AA /$ second.

Table 4-5 Growth conditions of CVO films.

\begin{tabular}{cccc}
\hline $\begin{array}{c}\text { Voltage } \\
(\mathbf{k V})\end{array}$ & $\begin{array}{c}\text { Pulse Rate } \\
(\mathbf{H z})\end{array}$ & $\begin{array}{c}\text { Ar Pressure } \\
(\mathbf{m T o r r})\end{array}$ & $\begin{array}{c}\text { Temperature } \\
\left({ }^{\circ} \mathbf{C}\right)\end{array}$ \\
\hline 15.4 & 9 & 7 & 650 \\
\hline
\end{tabular}

\subsubsection{Structure and Morphology}

CVO films were grown on STO substrates in a layer-by-layer mode with film thicknesses up to $15 \mathrm{~nm}$. The CVO films maintained good step-terrace structures with a 
step height equivalent to one unit cell's lattice spacing around 4 Å. As shown in Fig. 4-6, the AFM topography of a $5.2 \mathrm{~nm}$ CVO film showed an atomically flat surface with RMS roughness of $\sim 0.15 \mathrm{~nm}$. It was also observed that the surface RMS roughness increased with increasing film thickness as the highly strained CVO film (lattice mismatch with STO substrate of $-3.6 \%$ ) began to relax through the formation of islands on the film surface to relieve strain energy. These islands resulted in a rougher film surface with dislocations down to the substrate. ${ }^{88}$ For a CVO film with a thickness greater than $40 \mathrm{~nm}$, the typical surface RMS roughness increased to $\sim 1 \mathrm{~nm}$.
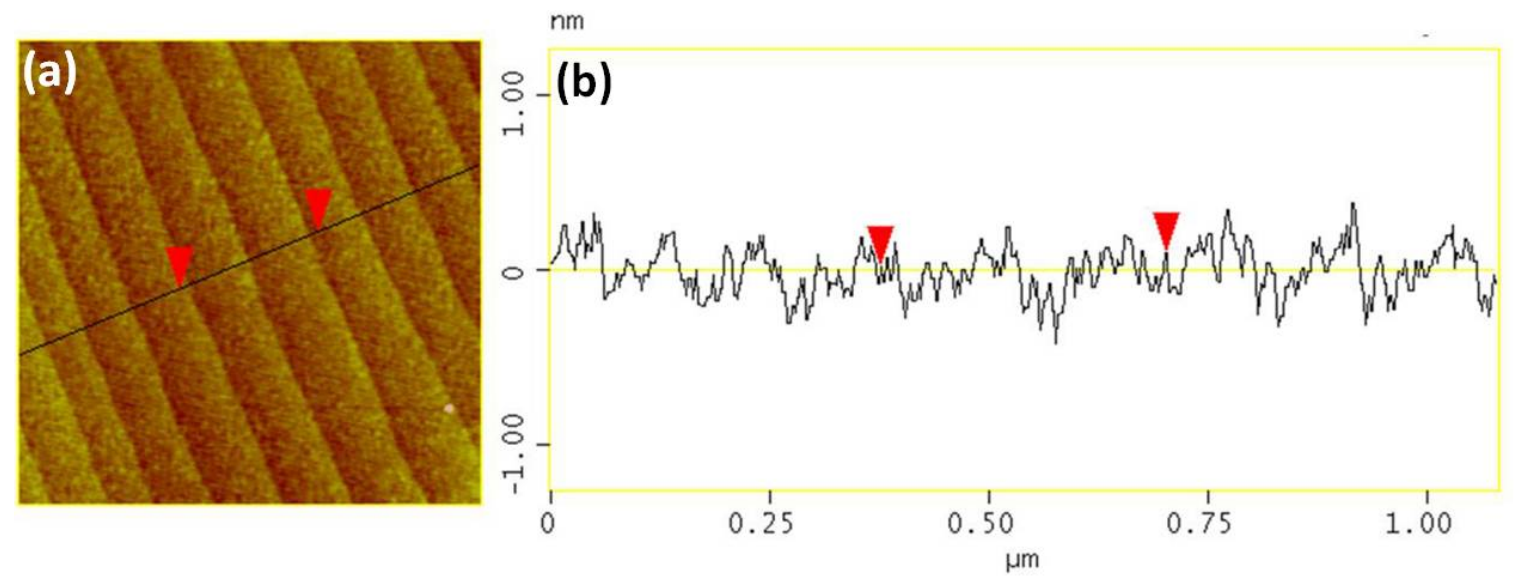

Figure 4-6 (a) AFM topography image and (b) line scan of a $5.2 \mathrm{~nm}$ CVO film grown on STO substrate (scan area: $1.0 \times 1.0 \mu \mathrm{m}^{2}$ ).

XRD measurements were performed to study the lattice structure of the CVO films. Fig. 4-7 shows the out-of-plane XRD scan of a CVO film with a thickness of 53 $\mathrm{nm}$. The wide-range scan showed a single-phase epitaxial CVO film grown on the STO substrate. The out-of-plane lattice parameter of the film was determined to be approximately $3.77 \AA$ based on the (002) film peak. This value is in agreement with the 
reported bulk value of CVO. ${ }^{50}$ This implies that the strain from the STO substrate was fully relaxed in the CVO film.

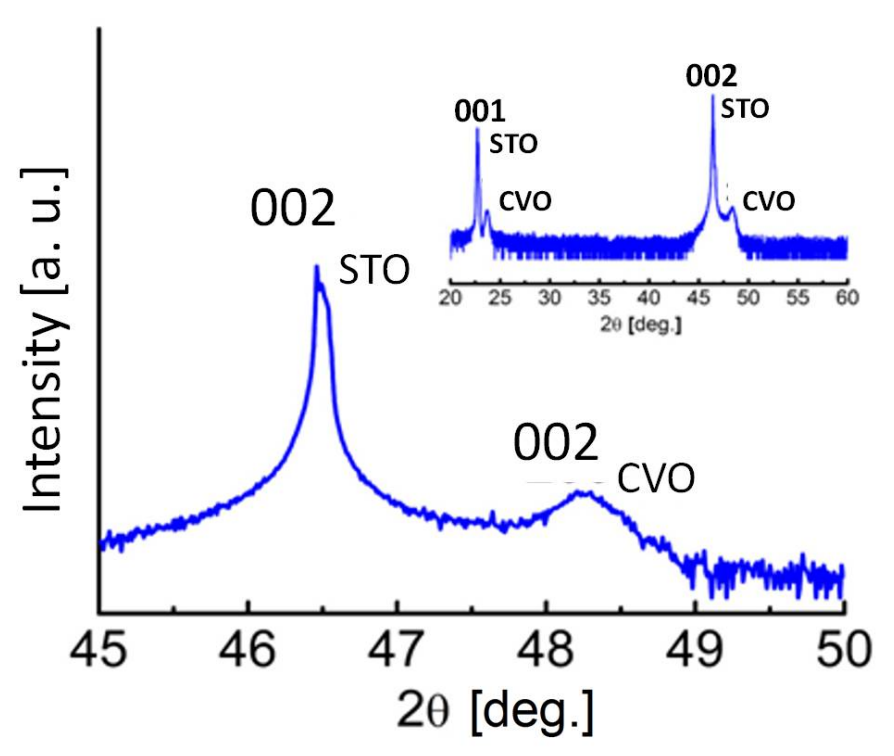

Figure 4-7 XRD scan around (002) reflection of a CVO film with thickness of $53 \mathrm{~nm}$. (Inset) Wide range scan showing that no secondary phases are present.

\subsubsection{Transport Properties}

To study the electrical transport properties of CVO films grown by PED, the temperature-dependent electrical resistivity and Hall effect measurements were performed on a CVO film with a thickness of $62 \mathrm{~nm}$. As shown in Fig. 4-8 (a), the resistivity of the CVO film was about $4.23 \times 10^{-4} \Omega \mathrm{cm}$ at room temperature and $3.35 \times$ $10^{-7} \Omega \mathrm{cm}$ at $2 \mathrm{~K}$. This low temperature residual resistivity is the lowest among all the reported values for single crystal and thin film samples. ${ }^{50,52,56,107}$ The resistivity ratio $\rho(300 \mathrm{~K}) / \rho(2 \mathrm{~K})$ was over 1200 , which is much larger than previous work on CVO thin 
films. ${ }^{56}$ Similar to the transport behaviors observed in the SVO films, the electrical resistivity of the CVO film followed the $\rho=\rho_{0}+A T^{2}$ relation corresponding to the Fermi liquid system in the temperature range from $2 \mathrm{~K}$ to $\sim 100 \mathrm{~K}$. The fitting yielded $\rho_{0}=3.17$ $\times 10^{-7} \Omega \mathrm{cm}$ and $A=1.22 \times 10^{-9} \Omega \mathrm{cm} / \mathrm{K}^{2}$. The resistivity deviated from the $T^{2}$ dependence when the temperature was raised above $50 \mathrm{~K}$. This occurrence is likely attributed to electron-phonon scattering. Hall measurements performed over the temperature range of 2-300 K are shown in Fig. 4-8 (b). Electrons were the majority carriers with a carrier concentration of $\sim 2 \times 10^{21} \mathrm{~cm}^{-3}$, which was one order of magnitude smaller than the theoretical value of $1.9 \times 10^{22} \mathrm{~cm}^{-3}$ assuming a simple one-band model. The carrier concentration showed a very weak temperature dependence over the entire temperature range that was investigated. In contrast, a very strong temperature dependence was observed for the electron mobility and that was responsible for the very large resistivity ratio $\rho(300 \mathrm{~K}) / \rho(2 \mathrm{~K})$ of $\mathrm{CVO}$. The electron mobility was $\sim 5 \mathrm{~cm}^{2} / \mathrm{Vs}$ at room temperature, and increased to over $6000 \mathrm{~cm}^{2} / \mathrm{Vs}$ at $2 \mathrm{~K}$, which was quite different from the low electron mobility observed in the SVO film grown on STO. Such a high electron mobility at low temperature is unexpected from a strongly correlated system, and may arise from the CVO/STO interface. 

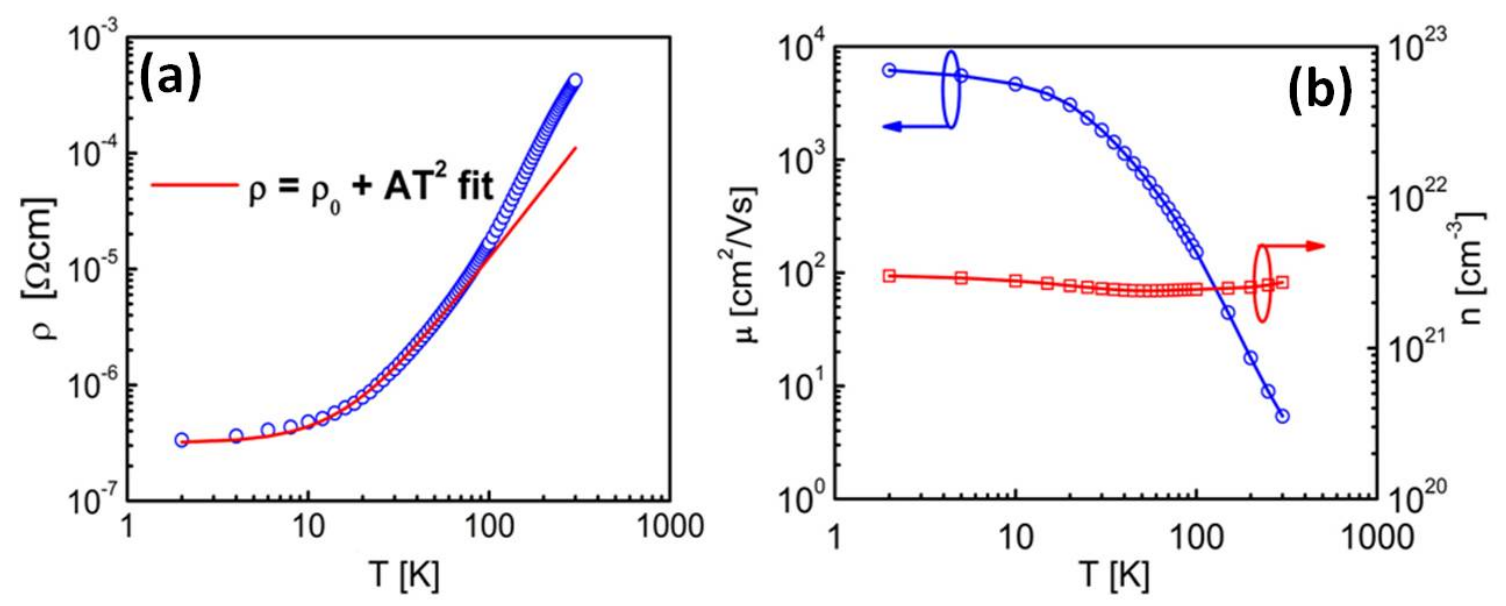

Figure 4-8 (a) Electrical resistivity vs. temperature for CVO film with thickness of $62 \mathrm{~nm}$. A $\rho=\rho_{0}+A T^{2}$ fit is also shown. (b) Temperature dependence of carrier mobility and carrier density of the same $62 \mathrm{~nm}$ CVO film.

\subsection{Summary}

In this chapter, the growth of high quality epitaxial $\mathrm{SrVO}_{3}(\mathrm{SVO})$ and $\mathrm{CaVO}_{3}$ (CVO) thin films has been demonstrated via the PED process. By carefully calibrating the growth conditions, both SVO and CVO thin films exhibited a layer-by-layer growth mode with smooth film surfaces. The electrical transport properties were explored in the vanadate films with thicknesses over $20 \mathrm{~nm}$. Similar to bulk vanadates, the films exhibited metallic behaviors followed the $T^{2}$ law corresponding to the Fermi liquid model. Such results have demonstrated the potential of PED in synthesizing high quality epitaxial oxide thin films despite the previous results in literature which show rough film surfaces due to the very high growth rate in PED. The successful growth of high quality epitaxial vanadate thin films enables us to study the transport properties of these materials via new approaches, such as dimensional confinement and chemical doping, 
providing great opportunities to control the metal-insulator transition (MIT) in these vanadate thin films. Methods of controlling the MIT will be discussed in the following chapters. 


\section{Chapter 5 Two-Dimensional Mott Insulators in Vanadates}

\subsection{Introduction}

Low dimensional strongly correlated electron systems have attracted much attention due to the intriguing electrical and magnetic properties arising from strong electron interactions. Experimental efforts have been made to explore quantum confined Mott materials such as $\mathrm{LaNiO}_{3}$ ultrathin films in order to address the role of reduced dimensionality on the transport properties. ${ }^{108,109}$ Recently, Mott insulating states induced in two-dimensional $\mathrm{SrVO}_{3}$ (SVO) quantum wells have been observed using in situ

photoemission spectroscopy (PES). ${ }^{70,71,72}$ I have demonstrated the ability to grow high quality epitaxial vanadate thin films using the PED technique in Chapter 4. In this chapter, we study the transport properties of SVO and CVO thin films as a function of film thickness ranging from tens of nanometers to a few nanometers. The temperature dependence of electrical resistivity revealed a MIT induced in two-dimensional vanadate ultrathin films with thicknesses of only a few nanometers, possible mechanisms behind the emergent MIT are discussed.

\section{2 $\mathrm{SrVO}_{3}$ Ultrathin Films}

\subsubsection{Structural Properties}

High quality epitaxial $\mathrm{SrVO}_{3}$ (SVO) thin films with thicknesses ranging from 25 $\mathrm{nm}$ to $3 \mathrm{~nm}$ were grown on (001)-oriented LSAT substrates via PED process. The lattice structures of SVO films were investigated as a function of film thickness. Out-of-plane 
XRD scans are shown in Fig. 5-1 (a), the (002) film peaks were found to progressively shift to higher angles as reducing film thicknesses to $6.5 \mathrm{~nm}$, and stayed at almost the same position with further reducing the thickness to $5.7 \mathrm{~nm}$. The calculated film out-ofplane lattice parameters are listed in Table 5-1. In Fig. 5-1 (b) and (c), the reciprocal space mapping (RSM) on (103) asymmetric reflection of the $25 \mathrm{~nm}$ and $15.3 \mathrm{~nm}$ films confirmed coherent growth of SVO films with the same in-plane lattice parameter as the underlying LSAT substrates $(a=3.868 \AA)$. RSM for the thinner films are not shown here owing to the low intensity of the film peaks.

The epitaxial SVO films grown on LSAT substrates were expected to be under inplane tensile strain due to the lattice mismatch of $-0.65 \%$. Studies reveal that the epitaxial strain can be accommodated up to a critical thickness $h_{c}$, and as the film thickness increases above $h_{c}$, the strain will be progressively relaxed through the formation of misfit dislocations at the interface. ${ }^{110}$ As observed from the film's out-of-plane lattice parameters listed in Table 5-1, the thicker films were more relaxed than the thinner ones The out-of-plane lattice parameter of the $25 \mathrm{~nm}$ SVO film was $3.846 \AA$, which is very close to the stoichiometric bulk SVO. ${ }^{57}$ Below $6.5 \mathrm{~nm}$, the SVO films were highly strained and showed an out-of-plane lattice parameter of $c=3.794 \AA$. The in-plane lattice parameters were expected to be $a=3.868 \AA$, since the films were constrained to be coherent to the in-plane LSAT substrate lattice. Assuming that the volume of a single unit cell was conserved, the cubic lattice parameters $a_{0}$ were calculated to be $3.843 \AA$ by using $a_{0}^{3}=a^{2} c$, which is also consistent with the lattice parameter of the stoichiometric bulk SVO. $^{57}$ 

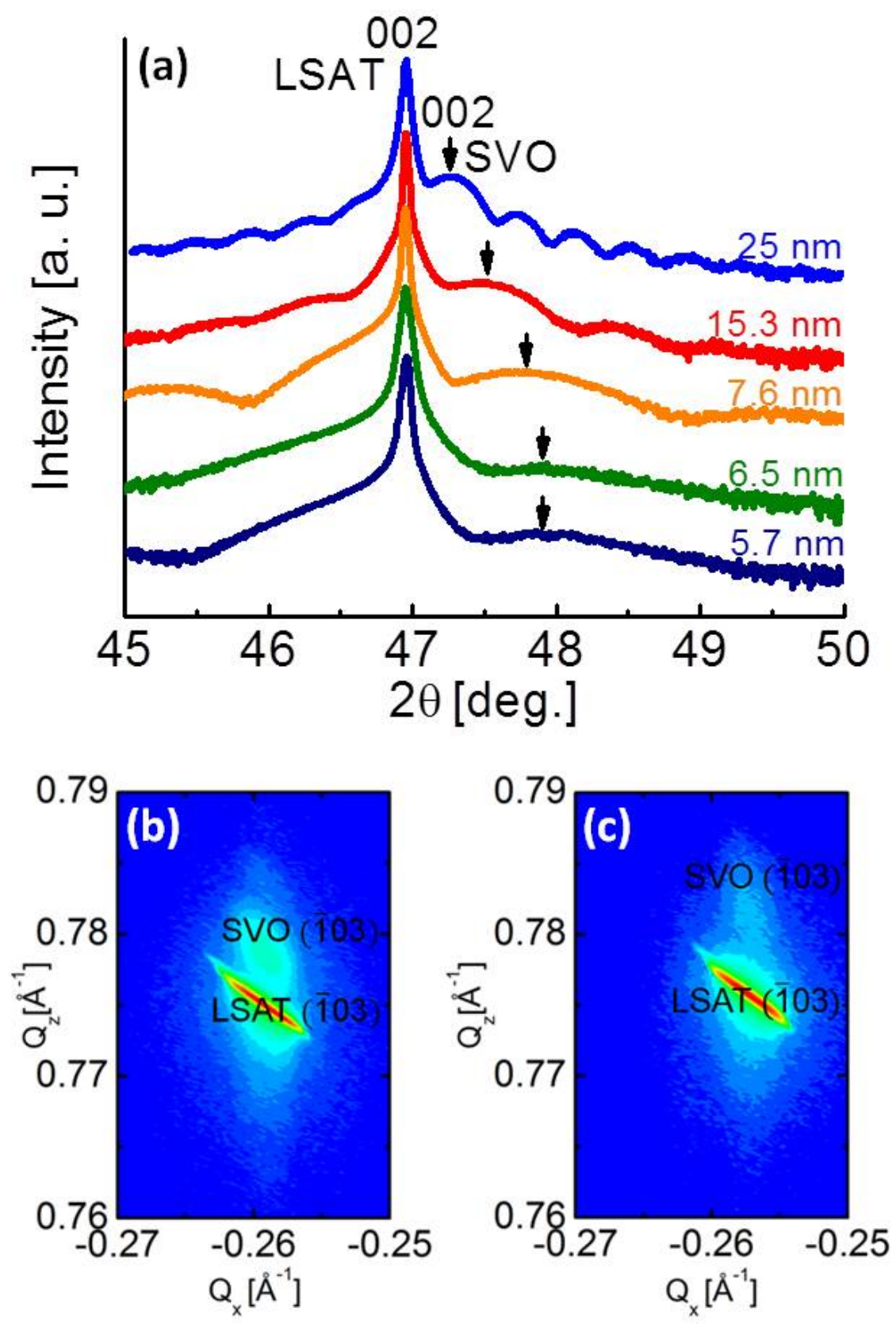

Figure 5-1 (a) XRD scans around (002) reflection of SVO films with various thicknesses, the SVO film peaks are indicated by the arrows. RSM on (103) reflection of (b) $25 \mathrm{~nm}$ and (c) $15.3 \mathrm{~nm}$ SVO films. 
Table 5-1 Out-of-plane lattice parameters of SVO films with various thicknesses.

\begin{tabular}{cc}
\hline Film Thickness & $\begin{array}{c}\text { Out-of-plane lattice parameter, } \\
c(\AA)\end{array}$ \\
\hline $\mathbf{2 5} \mathbf{~ n m ~ ( 6 5 ~ M L ) ~}$ & 3.846 \\
$\mathbf{1 5 . 3} \mathbf{~ n m ~ ( 4 0 ~ M L ) ~}$ & 3.829 \\
$\mathbf{7 . 6} \mathbf{~ n m ~ ( 2 0 ~ M L ) ~}$ & 3.809 \\
$\mathbf{6 . 5} \mathbf{~ n m ~ ( 1 7 ~ M L ) ~}$ & 3.794 \\
$\mathbf{5 . 7} \mathbf{~ m m ~ ( 1 5 ~ M L ) ~}$ & 3.794 \\
\hline
\end{tabular}

\subsubsection{Transport Properties}

Fig. 5-2 shows the electrical resistivity as a function of temperature for SVO films with thicknesses of $25 \mathrm{~nm}$ to $3 \mathrm{~nm}$. Reducing film thickness caused an increase in film resistivity in the entire temperature range. As discussed in Chapter 4, clear metallic behavior was observed in thicker SVO films with thickness of $25 \mathrm{~nm}$ and $15.3 \mathrm{~nm}$. For the SVO ultrathin films with thicknesses below $6.5 \mathrm{~nm}$, the films exhibited a temperature driven MIT characterized by a resistivity upturn at a transition temperature $T_{M I T}$ (shown with arrows in Fig. 5-2 (a)-(d)).

The transport data of the SVO films in this study is listed in Table 5-2. Above $T_{M I T}$, the resistivity as a function of temperature for the SVO ultrathin films below $6.5 \mathrm{~nm}$ $(\sim 17 \mathrm{ML})$ showed metallic behavior. The resistivity decreased with the decreasing temperature, indicating that the film was still continuous when the thickness was reduced to $3 \mathrm{~nm}(\sim 8 \mathrm{ML})$. In this metallic regime, the temperature dependence of resistivity still followed the $T^{2}$ law as an increase in the estimated values of $\rho_{0}$ and A was observed with 
decreasing film thickness. The resistivity reached a minimum at $T_{M I T}$. As the temperature was lowered further from $T_{M I T}$, the resistivity gradually increased as the films became insulating. As shown in the inset of Fig. 5-2 (a), the $T_{M I T}$ shifted to higher temperature by reducing film thickness. The $T_{M I T}$ was found to be at $50 \mathrm{~K}$ for the $6.5 \mathrm{~nm}$ film, $120 \mathrm{~K}$ for the $5.7 \mathrm{~nm}$ film, and $205 \mathrm{~K}$ for the $3 \mathrm{~nm}$ film. Near room temperature, an insulating SVO film is predicted to have a thickness of 2-3 ML which is consistent with the band structure observed by PES. ${ }^{70}$ In the insulating regime of the SVO ultrathin films with thickness less than $6.5 \mathrm{~nm}$, the temperature dependence of conductivity below $30 \mathrm{~K}$ followed the $\exp \left[-\left(T_{0} / T\right)^{1 / 4}\right]$ law that is consistent with Mott's variable range hopping (VRH) model (insets of Fig. 5-2 (b)-(d)). This behavior might be associated with the disorder-induced localized states at the Fermi level, or the percolation hopping between the granules as the films became very thin. ${ }^{111,112}$ 

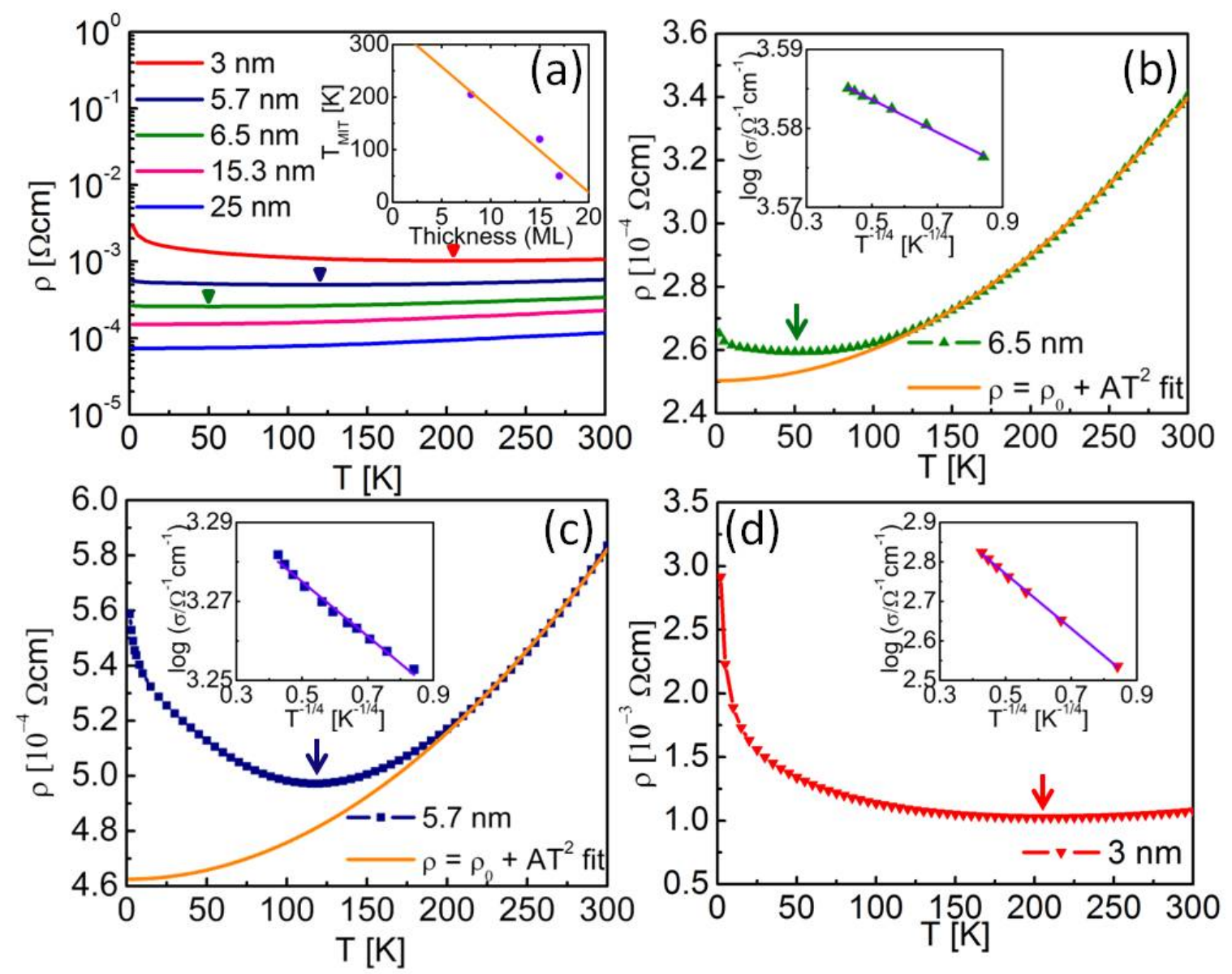

Figure 5-2 (a) Electrical resistivity vs. temperature for SVO films with thickness ranging from $3 \mathrm{~nm}$ to $25 \mathrm{~nm}$. Arrows indicate the local minima of resistivity. (Inset) $T_{M I T}$ vs. film thickness with a linear fit to the data. Temperature dependence of electrical resistivity on a different scale for SVO films with thicknesses of (b) $6.5 \mathrm{~nm}$, (c) $5.7 \mathrm{~nm}$ and (d) $3 \mathrm{~nm}$. (Inset) Logarithm of conductivity below $30 \mathrm{~K}$ as a function of $T^{1 / 4}$ for the same (b) $6.5 \mathrm{~nm}$, (c) $5.7 \mathrm{~nm}$ and (d) $3 \mathrm{~nm}$ SVO films. A linear fit to the data is also shown. 
Table 5-2 Transport properties of SVO films with various thicknesses deposited on the LSAT substrates.

\begin{tabular}{|c|c|c|c|c|c|c|}
\hline Film Thickness & $\begin{array}{r}\rho(300 \mathrm{~K}) \\
(\Omega \mathrm{cm})\end{array}$ & $\begin{array}{l}\rho(2 \mathrm{~K}) \\
(\Omega \mathrm{cm})\end{array}$ & $\begin{array}{l}T_{M I T} \\
(\mathrm{~K})\end{array}$ & $\begin{array}{c}\rho\left(T_{M I T}\right) \\
(\Omega \mathrm{cm})\end{array}$ & $\begin{array}{c}\rho_{0} \\
(\Omega \mathrm{cm})\end{array}$ & $\begin{array}{c}A \\
\left(\Omega \mathrm{cm} / \mathrm{K}^{2}\right)\end{array}$ \\
\hline $25 \mathrm{~nm}$ (65 ML) & $1.17 \times 10^{-4}$ & $7.37 \times 10^{-5}$ & - & - & $7.35 \times 10^{-5}$ & $4.95 \times 10^{-10}$ \\
\hline $15.3 \mathrm{~nm}(40 \mathrm{ML})$ & $2.30 \times 10^{-4}$ & $1.52 \times 10^{-4}$ & - & - & $1.51 \times 10^{-4}$ & $8.79 \times 10^{-10}$ \\
\hline $6.5 \mathrm{~nm}$ (17 ML) & $3.41 \times 10^{-4}$ & $2.65 \times 10^{-4}$ & 50 & $2.59 \times 10^{-4}$ & $2.50 \times 10^{-4}$ & $9.93 \times 10^{-10}$ \\
\hline $5.7 \mathrm{~nm}$ (15 ML) & $5.84 \times 10^{-4}$ & $5.59 \times 10^{-4}$ & 120 & $4.97 \times 10^{-4}$ & $4.62 \times 10^{-4}$ & $1.33 \times 10^{-9}$ \\
\hline 3 nm (8 ML) & $1.08 \times 10^{-3}$ & $2.91 \times 10^{-3}$ & 205 & $1.02 \times 10^{-3}$ & $9.40 \times 10^{-4}$ & $1.49 \times 10^{-9}$ \\
\hline
\end{tabular}

To understand the driving force behind the observed MIT induced in the SVO ultrathin films, magneto-transport measurements were performed to investigate the possible electron localization effects. The magneto-transport properties of the SVO ultrathin films turn out to be very different from the $\mathrm{LaNiO}_{3}$ ultrathin films, in which the MIT was driven by localization. ${ }^{108}$

In Fig. 5-3 (a), a small positive magnetoresistance (MR) in a perpendicular magnetic field was observed in the insulating regime of the SVO ultrathin film at the temperatures below $T_{M I T}$. The MR increased with decreasing temperature as the film became more insulating, and the MR was found to be proportional to $B^{2}$ which can be attributed to the Lorentz contribution. ${ }^{113}$ The observed positive MR is somewhat unexpected from electron localization, since the magnetic field suppresses the coherent interference for localization and results in a negative MR. ${ }^{114,115}$ Also, the Hall measurements of the same SVO ultrathin film are shown in Fig. 5-3 (b). The carriers 
were electrons as observed in the thick SVO film, as the temperature was lowered from room temperature, the electron mobility slightly increased while the concentration slightly decreased and stayed $\sim 7 \times 10^{21} \mathrm{~cm}^{-3}$ below $50 \mathrm{~K}$. However, in a disordered interacting electron system, the Hall coefficient was found to increase logarithmically at a rate equal to twice that of the resistivity at low temperature. ${ }^{115}$ The temperature dependence of the carrier concentration observed in the SVO ultrathin films did not at all exhibit that dependence. Moreover, previous studies show that, in a disordered electronic system, the effect of electron-electron interactions leads to a positive MR since the magnetic field suppresses two types of the hopping process in the presence of the intrasite interaction. It has also been demonstrated that the interplay of electron-electron interactions and localization results in the competition of positive and negative contributions to the field dependence of the MR. ${ }^{116,117,118}$ Based on the sign of MR and the temperature dependence of the carrier concentration and the mobility, we conclude that the dominant driving force behind the MIT induced in SVO ultrathin films is likely electron-electron interactions other than localization, while the magneto-transport behaviors at high magnetic fields may fully elicit the effect of the localization. 

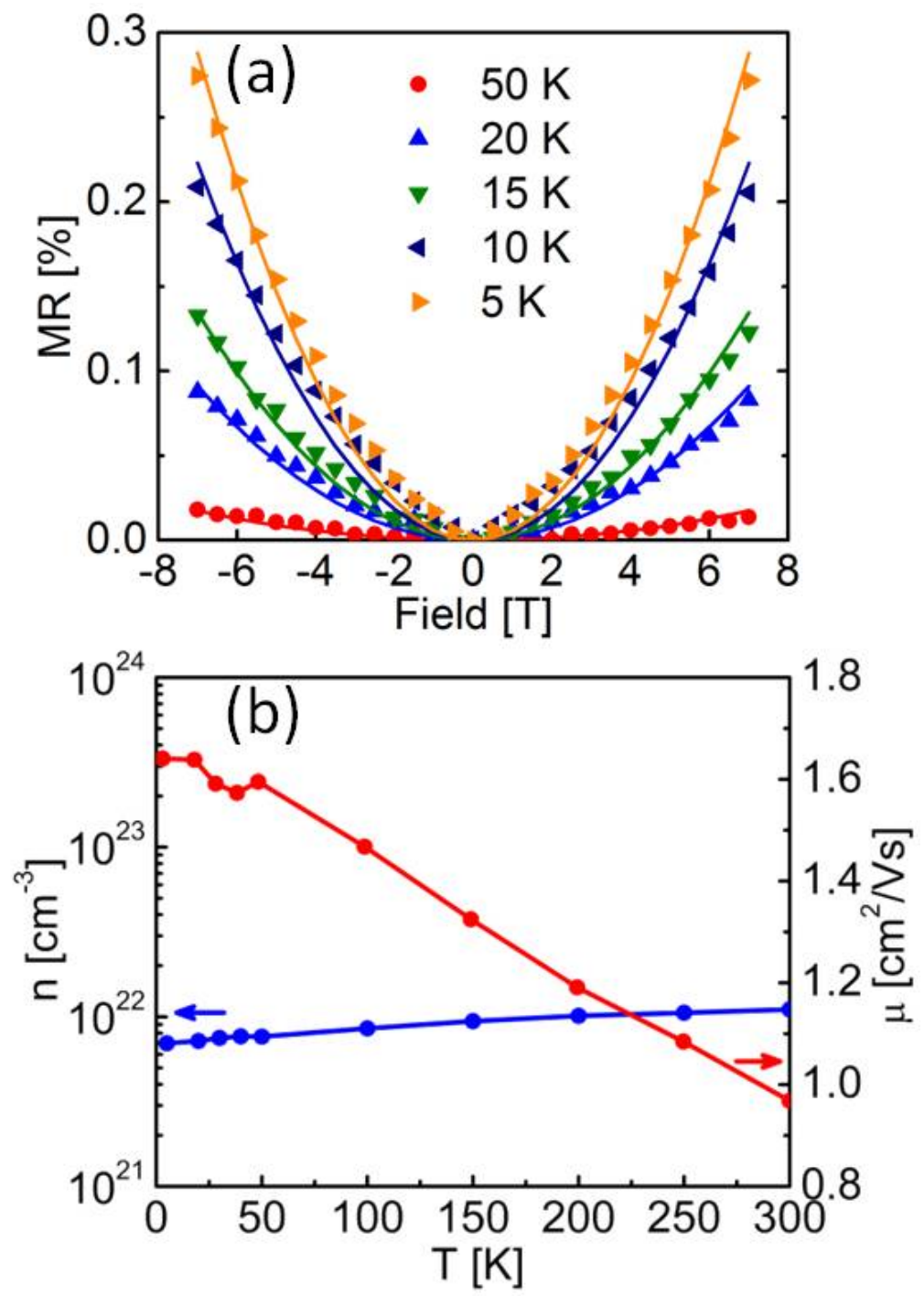

Figure 5-3 (a) Normalized out-of-plane MR of the $5.7 \mathrm{~nm} \mathrm{SVO}$ ultrathin film between 5 and $50 \mathrm{~K}$ with the field up to $7 \mathrm{~T}$. The MR is proportional to $B^{2}$ and the parabolic fits at each temperature are also shown in solid lines. (b) Temperature dependence of carrier density and mobility from Hall measurements of the same $5.7 \mathrm{~nm}$ SVO ultrathin film. 


\section{3 $\mathrm{CaVO}_{3}$ Ultrathin Films}

\subsubsection{Transport Properties}

The transport properties of epitaxial $\mathrm{CaVO}_{3}(\mathrm{CVO})$ films with thicknesses ranging from $62 \mathrm{~nm}$ to $2 \mathrm{~nm}$ were investigated in a similar way to SVO films. The CVO films were grown on (001)-oriented $\mathrm{SrTiO}_{3}$ (STO) substrates by PED. A $2.5 \mathrm{~nm} \mathrm{STO}$ capping layer was then deposited in-situ to protect the film, owing to the fact that CVO is metastable in air at room temperature. ${ }^{120}$ The sheet resistances of CVO films with different thicknesses are plotted as a function of temperature in Fig. 5-4. For comparison, a piece of STO substrate annealed at the temperature of deposition in the same environment was also characterized. The sheet resistance was $\sim 2 \times 10^{4} \Omega /$ sq. and shows no significant temperature dependence, indicating that the STO substrate was not reduced. For assessing the effect of the capping STO layer, we measured the single 18 nm STO film (dashed line) that demonstrated semiconducting behavior and found a very weak temperature dependence of the sheet resistance. Thus, we concluded that the transport of the STO substrate and the capping STO layer had no significant effects on the transport properties of our CVO samples despite previous reports on the metallic behaviors of reduced STO films. ${ }^{121,122}$

As discussed in Chapter 4, clear metallic behavior was observed in thick CVO films with thicknesses over $8 \mathrm{~nm}$. As shown in Fig. 5-4, the sheet resistance of a $62 \mathrm{~nm}$ CVO film was $68.4 \Omega /$ sq. at room temperature and decreased to $0.05 \Omega / \mathrm{sq}$. at $2 \mathrm{~K}$. Similarly, the CVO film with a thickness of $8.5 \mathrm{~nm}$ had a sheet resistance of $96.9 \Omega / \mathrm{sq}$. at room temperature, and that decreased to $1.3 \Omega / \mathrm{sq}$. at $2 \mathrm{~K}$. For CVO ultrathin films with 
thicknesses below $4 \mathrm{~nm}$, a temperature driven MIT was observed. The two CVO ultrathin films exhibited metallic behavior above the transition temperature. The sheet resistance of the $4 \mathrm{~nm} \mathrm{CVO} \mathrm{film} \mathrm{decreased} \mathrm{from} 182.3 \Omega /$ sq. at room temperature to $180.1 \Omega / \mathrm{sq}$. at $285 \mathrm{~K}$, and for the $2 \mathrm{~nm} \mathrm{CVO}$ film, the sheet resistance decreased from $341.8 \Omega / \mathrm{sq}$. at room temperature to $202.9 \Omega / \mathrm{sq}$. at $225 \mathrm{~K}$, the metallic behavior confirmed that the film was still continuous when the thickness was reduced to $2 \mathrm{~nm}(5 \mathrm{ML})$. As the temperature was lowered from the transition temperature, the sheet resistance of the two films gradually increased to $\sim 10^{4} \Omega /$ sq. at $\sim 100 \mathrm{~K}$ as the films became insulators. The sheet resistance of the insulating phases did not vary much as the temperature was decreased further.

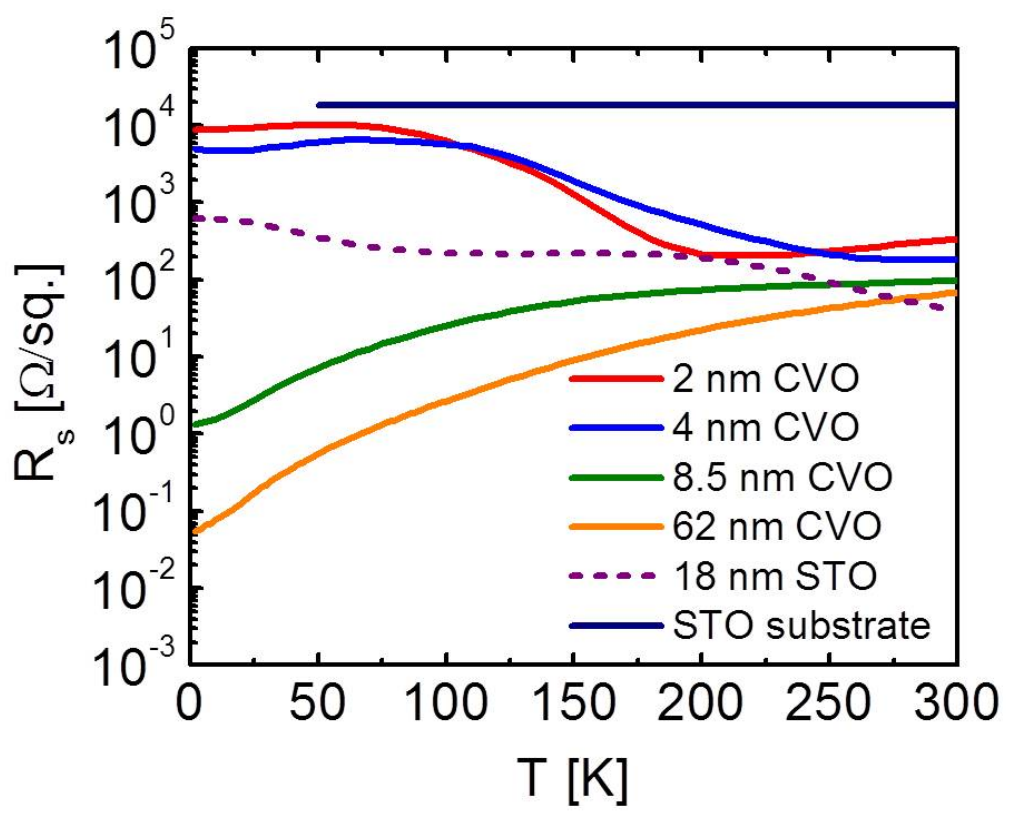

Figure 5-4 Sheet resistance vs. temperature for CVO films with thickness of 2, 4, 8.5, $62 \mathrm{~nm}$, STO film with thickness of $18 \mathrm{~nm}$, and STO substrate annealed at the temperature of deposition in the same environment. 
To further characterize the MIT in CVO ultrathin films with thicknesses of $2 \mathrm{~nm}$ and $4 \mathrm{~nm}$, I-V measurements were performed with a current that was varied linearly between $\pm 1 \mu \mathrm{A}$ in the temperature range of 2-100 K. As shown in the inset of Fig. 5-5, the measurements were performed using a four probe geometry. Two well-defined circular shaped contacts with radii of $150 \mu \mathrm{m}$ separated by $120 \mu \mathrm{m}$ were made in the center of the samples. The contacts were composed of $\mathrm{Al}(40 \mathrm{~nm}) / \mathrm{Ni}(20 \mathrm{~nm}) / \mathrm{Au}(150$ $\mathrm{nm}$ ) deposited by electron beam evaporation. For both of the CVO ultrathin films, the insulating phase was only observed in a narrow current range below $0.1 \mu \mathrm{A}$. Fig. 5-5 shows a representative contour plot of $\mathrm{dV} / \mathrm{dI}$ obtained from $\mathrm{I}-\mathrm{V}$ measurements of the 2 $\mathrm{nm}$ CVO film. The electric triggering of the MIT in CVO ultrathin films may result from two mechanisms: One is Joule heating, and the other is current-injection or electric-fieldbias induced carrier concentration change. ${ }^{123,124}$ Such a current-induced MIT in CVO ultrathin films could be eventually applied as very low power switching devices.

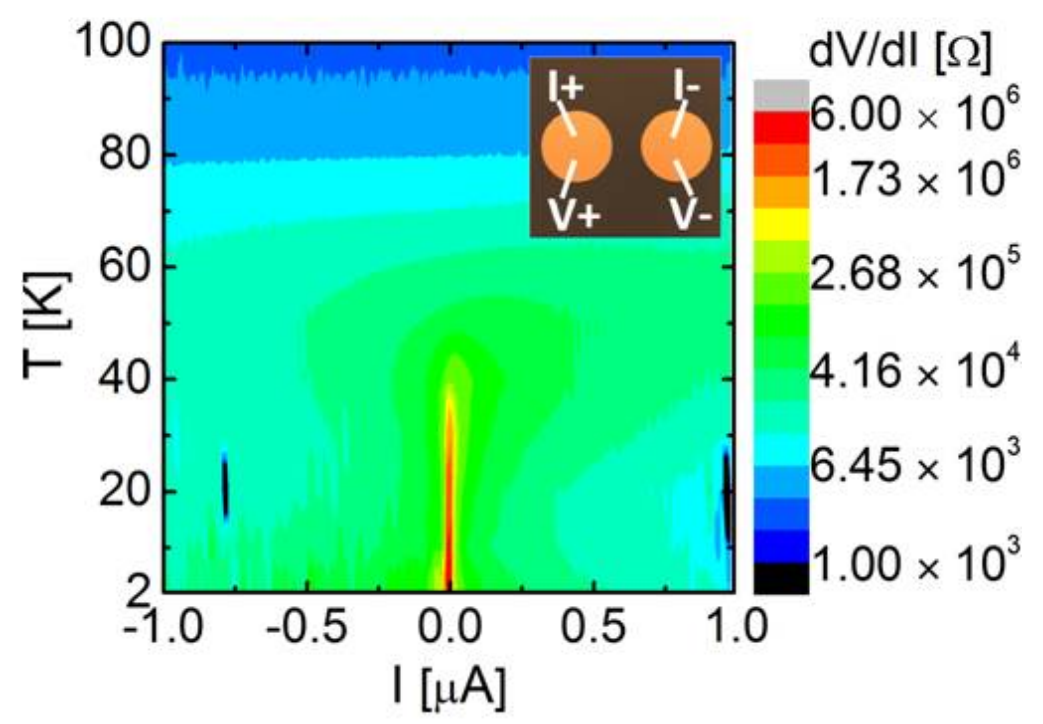

Figure 5-5 I-V measurements of $2 \mathrm{~nm}$ CVO film in the temperature range of 2-100 K. (Inset) Optical picture of the electrical contacts configuration. 


\subsubsection{Electronic Structures}

The electronic structures of the CVO films were examined by x-ray absorption spectroscopy (XAS). XAS measurements at the V and Ca $L$-edges in both TEY and TFY modes indicate a substantially modified electronic structure in both thick and ultrathin CVO films compared with the bulk. For reference, XAS spectra of a single-crystal CVO provided by Ravi Singh and Geetha Balakrishnan at University of Warwick are also shown in Fig. 5-6, which agree well with previous XAS measurements of polycrystalline CVO. ${ }^{125}$ However, at the V L-edge (Fig. 5-6 (a)) neither thick nor ultrathin CVO films resemble the single crystal spectra. In particular, the peak position of the $\mathrm{V} L_{3}$ edge was found to be $\sim 2 \mathrm{eV}$ lower in energy for the thicker $46 \mathrm{~nm}$ film, and the shape of the spectrum is in much closer agreement with $\mathrm{V}^{3+}$ spectra recorded in the more localized perovskite $\mathrm{LaVO}_{3}{ }^{126}$ In the TEY signal, extra weight at $\sim 518 \mathrm{eV}$ may indicate the presence of $\mathrm{V}^{4+} / \mathrm{V}^{5+}$ species at the $\mathrm{STO} / \mathrm{CVO}$ interface, owing to the shorter penetration depth of the TEY decay processes. However, for the ultrathin $4 \mathrm{~nm}$ film, the broad peaks in both TEY and, in particular, TFY suggest a broad mixture of $\mathrm{V}^{3+} / \mathrm{V}^{4+} / \mathrm{V}^{5+}$ species in the CVO layer, with overall charge state close to $4+$. The closer correspondence of the interface-sensitive TEY and bulk-sensitive TFY indicates a more defined twodimensional CVO layer in this ultrathin film. In Fig. 5-6 (b), the Ca $L$-edge spectra confirm that $\mathrm{Ca}$ retains its $\mathrm{Ca}^{2+}$ valence state in simple cubic symmetry for both films, ${ }^{127,128}$ which is consistent with the 12 -fold coordination of $\mathrm{Ca}$ in the perovskite structure of CVO. 

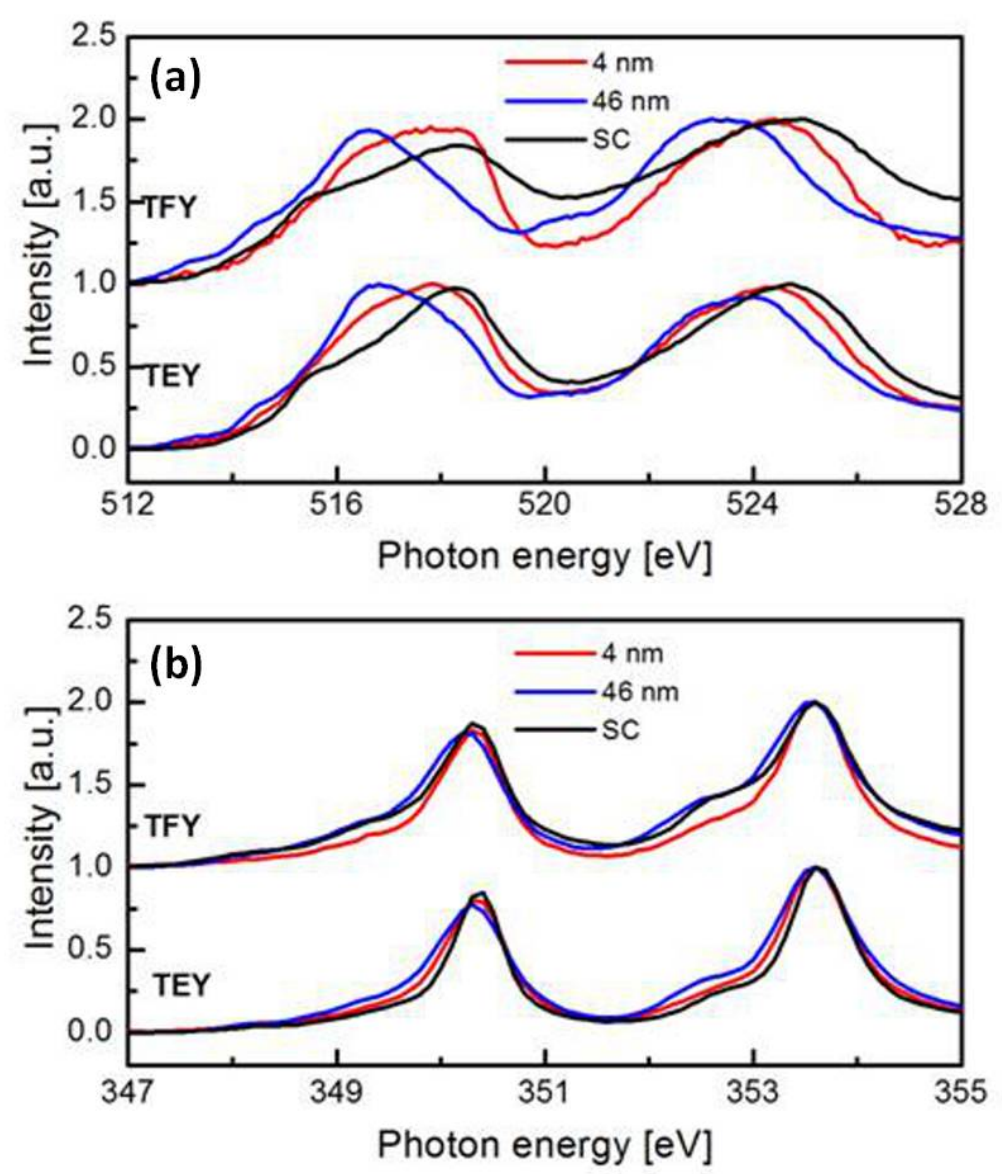

Figure 5-6 XAS spectra measured at (a) V L-edge and (b) Ca $L$-edge for CVO single crystal (SC) reference and STO capped CVO films with thickness of $4 \mathrm{~nm}$ and $46 \mathrm{~nm}$.

\subsection{MIT Mechanism}

The emergence of the MIT induced in vanadate ultrathin films could be attributed to a dimensional crossover from a three-dimensional metal to a two-dimensional Mott insulator. As discussed in Chapter 2, in a bandwidth-controlled MIT (BC-MIT), the system is determined by the $U / W$ ratio. ${ }^{4}$ The bandwidth $W$ is proportional to the number of available orbital states and thus highly sensitive to the film thickness. The atomic 
coordination at the interface and surface decreases with decreasing film thickness, resulting in a reduction of the effective bandwidth $W .^{70}$ According to the Hubbard model as illustrated in Fig. 5-7, a system with small $U / W$ (wide bandwidth $W$ ) corresponds to a metallic phase, in which the fully occupied lower Hubbard band (LHB) and the unoccupied upper Hubbard band (UHB) overlap. As the bandwidth $W$ decreases, the splitting between LHB and UHB gradually increases, and eventually evolves into a band gap at $E_{F}$ corresponding to a Mott insulator. Such a BC-MIT has also been observed at the surface of single-crystal CVO and SVO with a thickness of a few nanometers. ${ }^{130,131}$ In these single-crystal vanadates, the surface and bulk responses of the photoemission spectrum indicated fundamentally different electronic structures. A strong enhancement of $U / W$ at the surface compared with the bulk was observed, which could be attributed to the reduced atomic coordination at the surface. The strong enhancement of $U / W$ results in an insulating state at the surface. ${ }^{130}$ 


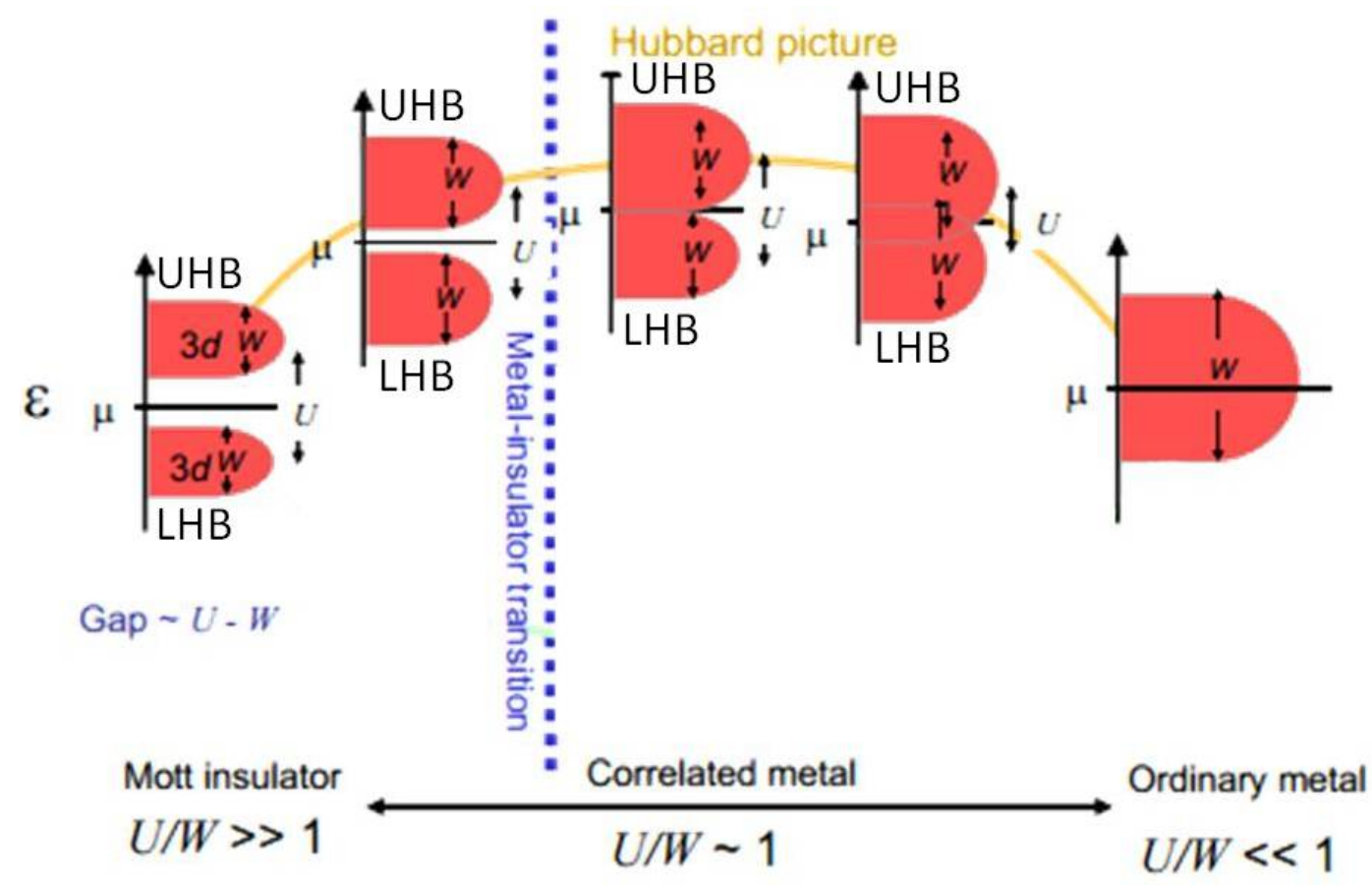

Figure 5-7 Schematic of the bandwidth-controlled MIT (BC-MIT). (Reproduced from Ref. 119)

The observed temperature driven MIT induced in vanadate ultrathin films shows a transition region as the films change from metallic states to insulating states from the dimensional crossover. The transition region may result from the thickness inhomogeneity, i.e. the inhomogeneity in the local electronic structure, in which thinner regions of the films have opened up a gap while thicker regions have not. This results in the coexistence of the metallic and insulating phases at the same temperature. When the metallic phase content reaches the percolative threshold, itinerant electrons can flow through the entire system. ${ }^{109,129}$

For the CVO films, in addition to the dimensional confinement, chemical doping from the STO capping layer may also need to be considered for its contribution to the 
induced MIT. It is unlikely that the MIT was induced by the substitution of $\mathrm{Sr}^{2+}$ ions for $\mathrm{Ca}^{2+}$ ions. This is because such a substitution would increase the V-O-V bond angle from $\sim 160^{\circ}$ to $\sim 180^{\circ}$, and the associated distortion increases the overlap between neighboring V $3 d$ orbitals which broadens the bandwidth. ${ }^{132,32}$ On the other hand, the study of the MIT in the bulk $\mathrm{CaV}_{1-x} \mathrm{Ti}_{x} \mathrm{O}_{3}$ system showed that the substitution of $\mathrm{Ti}^{4+}$ ions for $\mathrm{V}^{4+}$ ions would perturb the periodic potential so as to trap electrons in Anderson-localized states below a mobility edge, and that induced a MIT with increasing $x$ in the range of $0.2<x<$ $0.4{ }^{120}$ Furthermore, the chemical doping of $\mathrm{Sr}^{2+}$ and $\mathrm{Ti}^{4+}$ ions would inevitably induce randomness in the CVO system, the broadened peak at V $L$-edge in XAS spectra as shown in Fig. 5-6 (a) also indicated broad mixture of $\mathrm{V}^{3+} / \mathrm{V}^{4+} / \mathrm{V}^{5+}$ species in the ultrathin CVO layer, these chemical disorder effects could also drive a MIT in the strongly correlated systems. ${ }^{133,134}$ Chemical doping effects in the vanadate thin films will be presented in the next chapter.

\subsection{Summary}

In this chapter, we have studied the transport properties of vanadate thin films as a function of film thickness, and demonstrated two-dimensional Mott insulators in the vanadate ultrathin films with thicknesses of only a few nanometers. The emergence of the observed MIT can be attributed to the dimensional crossover from a three-dimensional metal to a two-dimensional Mott insulator, as the resulting reduction in the effective bandwidth $W$ opens a bandgap at the Fermi level. 
For SVO films grown on LSAT substrates, the transport study showed that thick SVO films $(\sim 25 \mathrm{~nm})$ exhibited metallic behavior. A temperature driven MIT was induced in SVO ultrathin films with thicknesses below $6.5 \mathrm{~nm}$. An increase in the transition temperature $T_{M I T}$ was observed with decreasing film thickness, $T_{M I T}$ was found to be at 50 $\mathrm{K}$ for the $6.5 \mathrm{~nm}$ film, $120 \mathrm{~K}$ for the $5.7 \mathrm{~nm}$ film and $205 \mathrm{~K}$ for the $3 \mathrm{~nm}$ film. At near room temperature, an insulating SVO film was predicted to have a thickness of 2-3 ML, which agrees with the band structure studied by PES. ${ }^{70}$ The magneto-transport study of the SVO ultrathin films showing MIT also confirmed that the dominant driving force behind the observed MIT is electron-electron interactions other than localization. Such a dimensional-crossover-driven MIT has also been observed in CVO ultrathin films grown on STO substrates with thicknesses below $4 \mathrm{~nm}$. XAS measurements indicated different electronic structures for thick and very thin films of CVO. Compared with the thick film $(\sim 60 \mathrm{~nm})$, thin films of CVO $(2-4 \mathrm{~nm})$ were more two-dimensional with the $\mathrm{V}$ charge state closer to $\mathrm{V}^{4+}$. 


\section{Chapter 6 MIT in $\operatorname{SrTi}_{1-x} V_{x} \mathrm{O}_{3}$ Thin Films}

\subsection{Introduction}

The bandfilling-controlled metal-insulator transition (MIT) can be induced in vanadates via aliovalent $\mathrm{A}$-site substitution such as in the $\mathrm{La}_{1-x} \mathrm{Ca}_{x} \mathrm{VO}_{3}{ }^{64,65}, \mathrm{Gd}_{1-}$ ${ }_{x} \mathrm{Ca}_{x} \mathrm{VO}_{3}{ }^{66}$, and $\mathrm{La}_{1-x} \mathrm{Sr}_{x} \mathrm{VO}_{3}{ }^{67,68,69}$ systems. For B-site substitution, $\mathrm{SrTi}_{1-x} \mathrm{~V}_{x} \mathrm{O}_{3}(\mathrm{STVO})$,

the solid solution comprised of a correlated metal $\mathrm{SrVO}_{3}(\mathrm{SVO})\left(3 d^{1}\right)$ and a band insulator $\mathrm{SrTiO}_{3}$ (STO) $\left(3 d^{0}\right)$ offers a very interesting system. Both SVO and STO are cubic perovskites with similar lattice constants $\left(a=3.843 \AA\right.$ for $\mathrm{SVO}^{57} ; a=3.905 \AA$ for STO). Since the element vanadium is next to titanium in the periodic table, the solid solution is expected to form over a wide range of compositions. In this chapter, I synthesize the STVO thin films via the combinational deposition and characterize the transport properties of the STVO thin films for $0 \leq x \leq 1$. The possible mechanisms behind the observed MIT will also be discussed, including the effects of electron correlation, lattice distortion, and disorder.

\subsection{Film Growth}

High-quality epitaxial $\mathrm{SrTi}_{1-x} \mathrm{~V}_{x} \mathrm{O}_{3}(\mathrm{STVO})$ thin films with thicknesses of $\sim 16 \mathrm{~nm}$ were deposited on (001)-oriented LSAT substrates $(a=3.868 \AA)$ via PED process. The STVO thin films were co-deposited from two individual ceramic targets using two electron-beam guns. In the case of $\mathrm{SrVO}_{3}(\mathrm{SVO})$, a $12 \mathrm{kV}$ potential in the electron-beam gun was applied to ablate a $\mathrm{Sr}_{2} \mathrm{~V}_{2} \mathrm{O}_{7}$ target. The deposition rate for $\mathrm{SVO}$ was found to be 
$0.006 \AA$ pulse which equates to $1.8 \AA / \mathrm{min}$ at a $5 \mathrm{~Hz}$ pulse rate. For $\mathrm{SrTiO}_{3}$ (STO), a stoichiometric STO target was used and the electron-beam gun was operated at $10 \mathrm{kV}$ to achieve a similar growth rate as SVO. The composition of the STVO thin films was controlled by varying the pulse rate for each of the two targets in the range of 1-5 Hz. All the films in this chapter were grown at a substrate temperature of $800{ }^{\circ} \mathrm{C}$ in a $10 \mathrm{mTorr}$ Ar atmosphere. The growth conditions are listed in Table 6-1.

Table 6-1 Growth conditions of STVO films.

\begin{tabular}{lcccc}
\hline & $\begin{array}{c}\text { Voltage } \\
(\mathbf{k V})\end{array}$ & $\begin{array}{c}\text { Pulse Rate } \\
(\mathbf{H z})\end{array}$ & $\begin{array}{c}\text { Ar Pressure } \\
(\mathbf{m T o r r})\end{array}$ & $\begin{array}{c}\text { Temperature } \\
\left({ }^{\circ} \mathbf{C}\right)\end{array}$ \\
\hline SVO & 12 & $1-5$ & 10 & 800 \\
STO & 10 & $1-5$ & 10 & 800 \\
\hline
\end{tabular}

\subsection{Structural Properties}

The film surface morphology was analyzed with AFM, which revealed that the growth of the STVO thin films on LSAT substrates was carried out in a layer-by-layer growth mode. Furthermore, all the films showed atomically flat surfaces with an RMS roughness of $\sim 0.2 \mathrm{~nm}$ as shown in the previous chapter.

Out-of-plane XRD scans are shown in Fig. 6-1 (a), which confirmed that the films are a single phase STVO solid solution system in the whole composition range of $0 \leq x \leq$ 1. The Kiessig fringes around the (001) and (002) diffraction peaks indicated coherent films with smooth interfaces. Since SVO has a smaller lattice parameter than STO, the STVO film peaks gradually shifted to the higher $2 \theta$ value with the increase of the 
vanadium concentration $x$. The calculated out-of-plane lattice parameters $c$ are listed in Table 6-2. The reciprocal space mapping on the (103) asymmetric reflection in Fig. 6-1 (c)-(i) also confirmed coherent growth of all the STVO $(0 \leq x \leq 1)$ thin films. The inplane lattice parameters $a$ were found to be $3.868 \AA$ for all the films, since the films were coherently strained to the in-plane lattice parameter of the LSAT substrates. As listed in Table 6-2, the unit cell volumes $V$ were calculated from both out-of-plane and in-plane lattice parameters. Following Vegard's law, ${ }^{135} V$ decreased linearly with increasing $x$ as shown in Fig. 6-1 (b).

Table 6-2 Lattice parameters of the STVO $(0 \leq x \leq 1)$ thin films. (For all the films, in-plane lattice parameters $a=3.868 \AA)$.

\begin{tabular}{cccc}
\hline $\mathbf{V}$ concentration, & Out-of-plane lattice parameter, & \multicolumn{2}{c}{ Unit cell volume, } \\
$\boldsymbol{x}$ & $\boldsymbol{c}(\mathbf{\AA})$ & $\boldsymbol{c} / \boldsymbol{a}$ & $\boldsymbol{V}\left(\mathbf{\AA}^{\mathbf{3}}\right)$ \\
\hline $\mathbf{1}$ & 3.829 & 0.990 & 57.287 \\
$\mathbf{0 . 8 3}$ & 3.847 & 0.995 & 57.557 \\
$\mathbf{0 . 6 7}$ & 3.879 & 1.003 & 58.035 \\
$\mathbf{0 . 5}$ & 3.926 & 1.015 & 58.739 \\
$\mathbf{0 . 3 3}$ & 3.973 & 1.027 & 59.442 \\
$\mathbf{0 . 1 7}$ & 4.013 & 1.037 & 60.040 \\
$\mathbf{0}$ & 4.023 & 1.040 & 60.190 \\
\hline
\end{tabular}



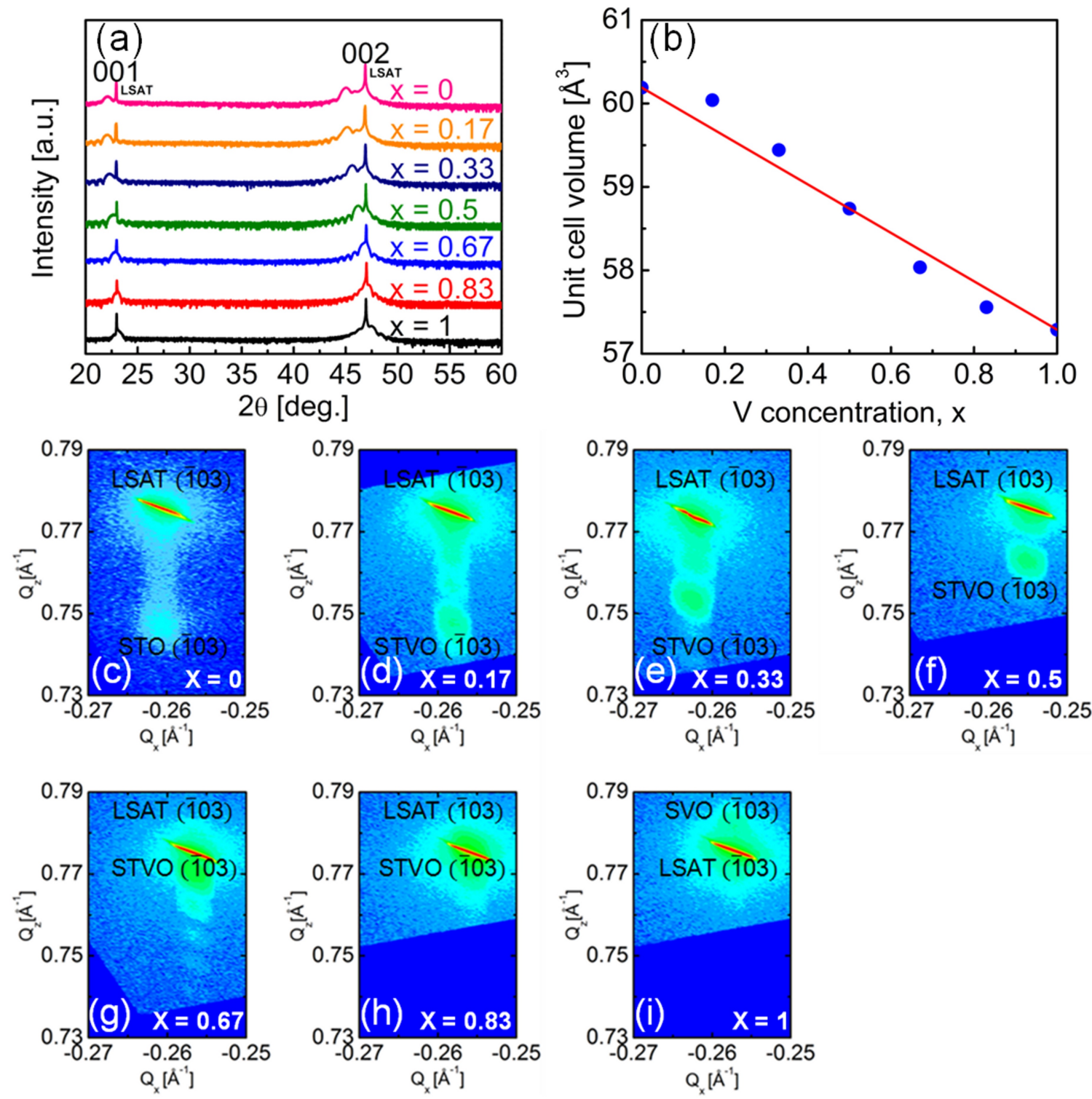

Figure 6-1 (a) XRD scans for the STVO $(0 \leq x \leq 1)$ thin films grown on LSAT (001) substrates. (b) Calculated unit cell volume as a function of $x$. The straight line represents the Vegard's law. (c)-(i) The reciprocal space mapping on (103) reflection for the same STVO thin films. 


\subsection{Transport Properties}

Fig. 6-2 (a) shows the electrical resistivity as a function of temperature for the STVO $(0.33 \leq x \leq 1)$ thin films in the temperature range of $2-300 \mathrm{~K}$. The $x=0.17$ and 0 films were too insulating for transport measurements. The resistivity data is listed in Table 6-3. Reducing the vanadium concentration $x$ caused an increase in film resistivity over the entire temperature range. The film with $x=0.67$ was found to lie at the boundary of the transition from a metallic to a semiconducting state and showed a temperature driven MIT. The films with higher vanadium concentration $(x=0.83$ and 1$)$ were metallic, and the ones with lower vanadium concentration $(x=0.33$ and 0.5$)$ were semiconducting. Also, room-temperature Hall effects were used to determine the carrier type and concentration. The carriers were found to be electrons and the carrier density decreased with the decrease of $x$ as shown in Fig. 6-2 (b).
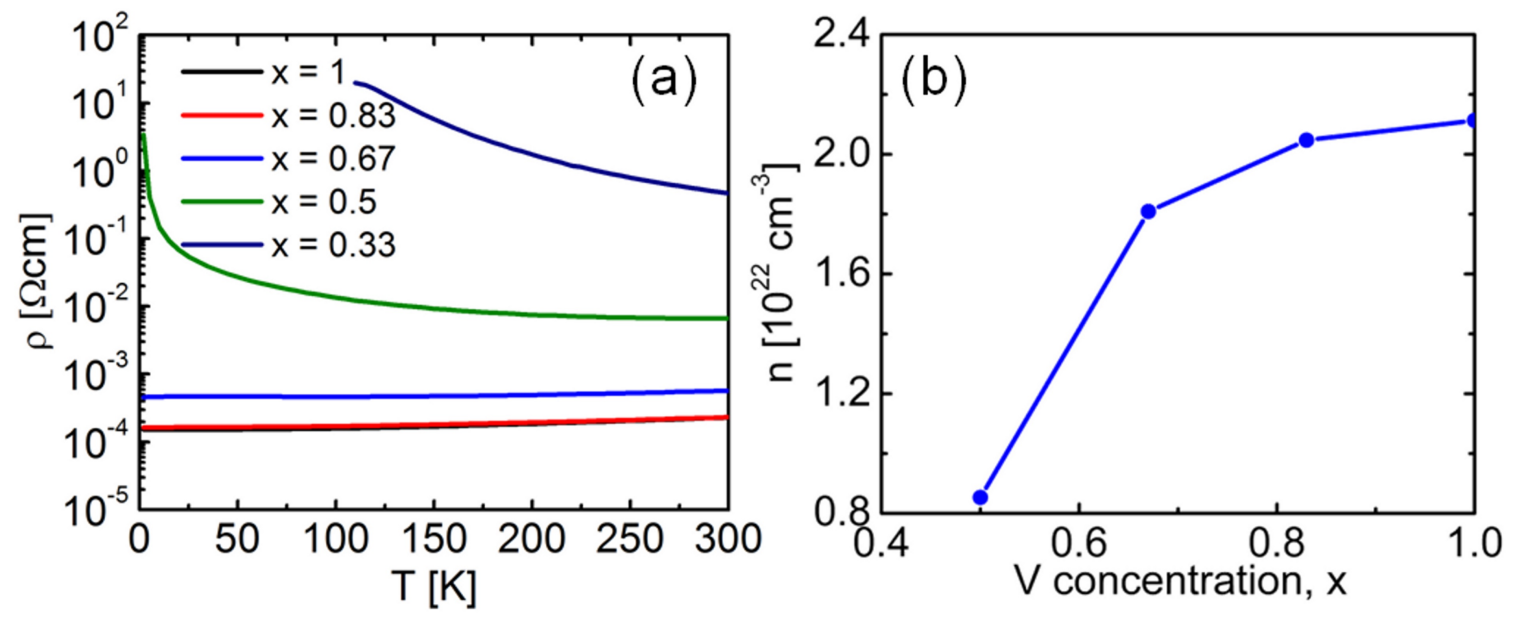

Figure 6-2 (a) Electrical resistivity vs. temperature for the STVO $(0.33 \leq$ $x \leq 1)$ thin films. (b) Carrier density from room-temperature Hall measurements for the STVO $(0.5 \leq x \leq 1)$ thin films. 
The detailed temperature dependence of the transport properties for the STVO $(0.33 \leq x \leq 1)$ thin films is shown in Fig. 6-3 (a)-(d). Both the $x=1$ and $x=0.83$ films exhibited metallic behaviors (Fig. 6-3 (a)), and the electrical resistivity of the $x=0.83$ film was slightly higher than that of the pure SVO film $(x=1)$. Over the entire temperature range of $2-300 \mathrm{~K}$, the electrical resistivity as a function of temperature followed the $\rho=\rho_{0}+A T^{2}$ relationship corresponding to a Fermi liquid model. The fitting parameters of $\rho_{0}$ and $A$ are listed in Table 6-3. In Fig. 6-3 (b), the $x=0.67$ film exhibited a temperature-driven MIT characterized by a resistivity upturn at a transition temperature $T_{M I T}$ of $95 \mathrm{~K}$ (indicated by the arrow). Above $T_{M I T}$, the $x=0.67$ film showed metallic behavior with the temperature dependence of resistivity still following the $T^{2}$ law. As listed in Table 6-3, an increase in the estimated values of $\rho_{0}$ was observed with decreasing $x$, and the $x=0.67$ film showed a much higher $A$ value than the $x=0.83$ and $x=1$ films. As the temperature was lowered below $T_{M I T}$, the resistivity of the $x=0.67$ film gradually increased, and then dropped a little at temperatures below $35 \mathrm{~K}$. Both the $x=0.5$ and $x=$ 0.33 films exhibited semiconducting behaviors. As plotted in Fig. 6-3 (c) and (d), the temperature dependence of conductivity followed the $\exp \left[-\left(T_{0} / T\right)^{1 / 4}\right]$ law, which is consistent with Mott's variable range hopping (VRH) model ${ }^{136}$ in the entire temperature range. Furthermore, a dramatic increase in the fitting parameter $T_{0}$ with decreasing $x$ was observed (Table 6-3). 

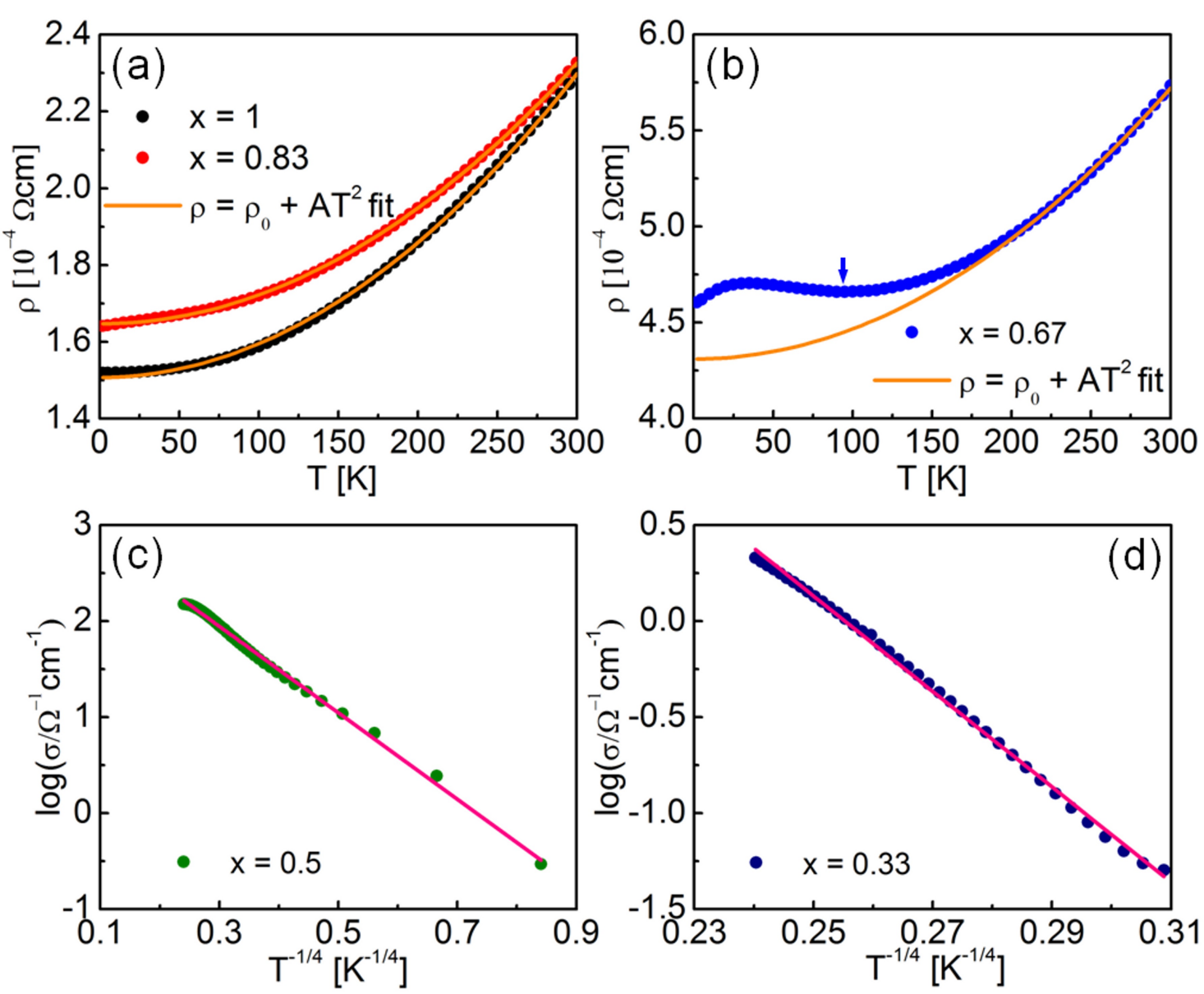

Figure 6-3 Temperature dependence of electrical resistivity for the STVO thin films, (a) $x=1$ and 0.83 , (b) $x=0.67, \rho=\rho_{0}+A T^{2}$ fits are also shown. Logarithm of conductivity as a function of $T^{-1 / 4}$ with a linear fit to the data, (c) $x=0.5,2 \mathrm{~K} \leq T \leq 300 \mathrm{~K}$, (d) $x=0.33,110 \mathrm{~K} \leq T \leq 300 \mathrm{~K}$.

Table 6-3 Transport properties of the STVO $(0.33 \leq x \leq 1)$ thin films.

\begin{tabular}{cccccc}
\hline $\begin{array}{c}\text { V concentration, } \\
\boldsymbol{x}\end{array}$ & $\begin{array}{c}\boldsymbol{\rho}(\mathbf{3 0 0} \mathbf{K}) \\
(\boldsymbol{\Omega c m})\end{array}$ & $\begin{array}{c}\boldsymbol{\rho}(\mathbf{2} \mathbf{K}) \\
(\boldsymbol{\Omega c m})\end{array}$ & $\begin{array}{c}\boldsymbol{\rho}_{\mathbf{0}} \\
(\mathbf{\Omega c m})\end{array}$ & $\begin{array}{c}A \\
\left(\mathbf{\Omega c m} / \mathbf{K}^{\mathbf{2}}\right)\end{array}$ & $\begin{array}{c}\boldsymbol{T}_{\mathbf{0}} \\
(\mathbf{K})\end{array}$ \\
\hline $\mathbf{1}$ & $2.30 \times 10^{-4}$ & $1.52 \times 10^{-4}$ & $1.51 \times 10^{-4}$ & $8.79 \times 10^{-10}$ & - \\
$\mathbf{0 . 8 3}$ & $2.33 \times 10^{-4}$ & $1.64 \times 10^{-4}$ & $1.65 \times 10^{-4}$ & $7.54 \times 10^{-10}$ & - \\
$\mathbf{0 . 6 7}$ & $5.73 \times 10^{-4}$ & $4.60 \times 10^{-4}$ & $4.31 \times 10^{-4}$ & $1.57 \times 10^{-9}$ & - \\
$\mathbf{0 . 5}$ & $6.65 \times 10^{-3}$ & 3.40 & - & - & $1.16 \times 10^{4}$ \\
$\mathbf{0 . 3 3}$ & 0.47 & $>20$ & - & - & $1.07 \times 10^{7}$ \\
\hline
\end{tabular}


A low temperature magneto-transport study was carried out to understand the transport mechanism in the STVO thin films. As shown in Fig. 6-4, magnetoresistance (MR) in a perpendicular magnetic field was measured for the $x=0.67$ film in the low temperature range of 2-30 K. A small positive MR was observed for all the temperatures. The MR increased with the decrease in temperature and the MR was found to be roughly proportional to $B^{2}$ at each temperature. The positive MR is inconsistent with the MIT induced by Anderson localization, i.e. the weak localization. It is expected that the magnetic field suppresses the coherent interference for the weak localization, and thereby results in a negative MR. ${ }^{114,115}$ It is well-known that electron-electron interactions play an important role in the Anderson-localized regime of the impurity band in doped semiconductors. ${ }^{6}$ The effects of electron-electron interactions on the MR in the Anderson-localized regime have been investigated. In the presence of a magnetic field, the interplay of the intrastate interaction and the energy dependence of localization lengths results in the competition of positive and negative contributions to the field dependence of the MR. ${ }^{116,118}$ Therefore, the positive MR observed in the STVO thin films may suggest that the effect of electron-electron interactions is not negligible and Anderson localization is not the only driving force behind the observed MIT. 


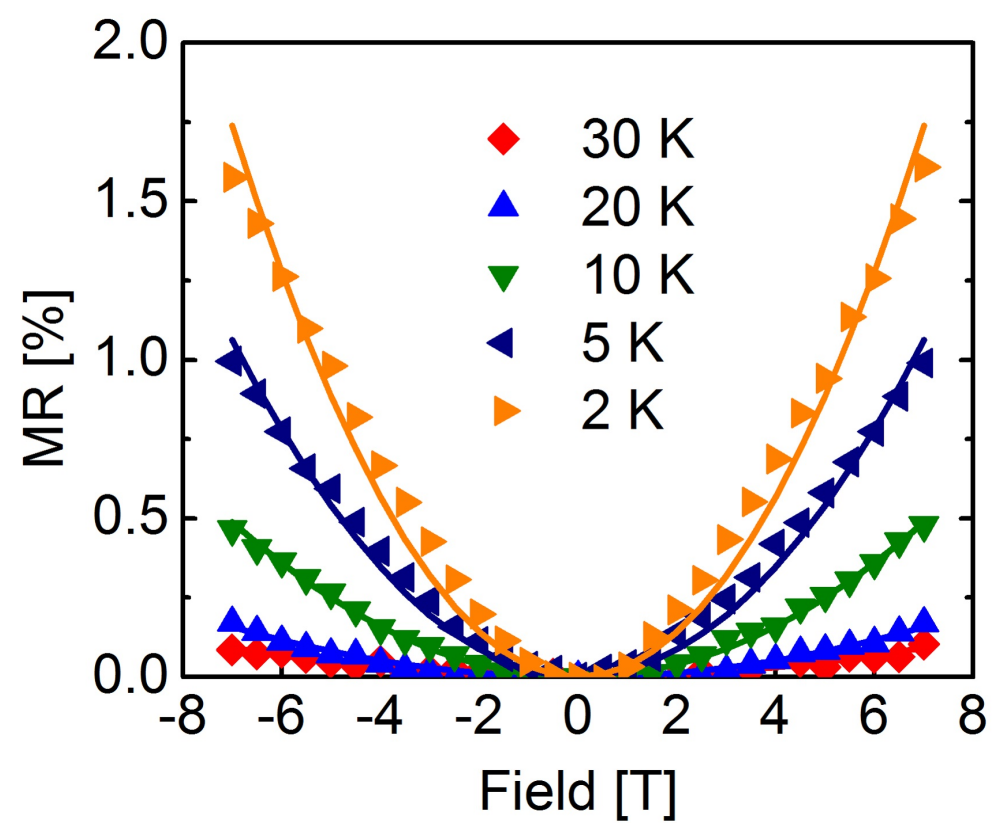

Figure 6-4 Normalized out-of-plane MR of the STVO $(x=0.67)$ thin film between $2 \mathrm{~K}$ and $30 \mathrm{~K}$ with the field up to $7 \mathrm{~T}$. The parabolic fits at each temperature are also shown in lines.

\subsection{MIT Mechanism}

Our observation of the MIT induced in the STVO $(x=0.67)$ thin film is in good agreement with the literature data for the bulk material. ${ }^{137,138}$ Tsuiki et al. ${ }^{137}$ reported a MIT in the $x \approx 0.6$ sample and VRH mechanisms for the samples with lower vanadium concentration $(x \leq 0.6)$. The high critical donor concentration $x$ for the MIT was interpreted by a very deep donor level assuming a Jahn-Teller distortion induced around the $\mathrm{V}^{4+}$ ion on the STO lattice. The local Jahn-Teller distortion makes the electrons selftrapped and strongly localized to the donor center. On the other hand, Hong et al. ${ }^{138}$ observed a temperature driven MIT in the $x=0.7$ sample with a transition temperature $T_{M I T}$ of $120 \mathrm{~K}$, which is quite similar to our result. However, a different conduction 
mechanism in the semiconducting region was observed by Hong et al. as the low temperature data of the $x=0.7$ sample can be scaled with a 3D weak localization model, and a model of Anderson localized states was proposed in which a MIT occurs where the mobility edge crosses the Fermi level. ${ }^{138}$ Moreover, the study of a similar system of $\mathrm{CaV}_{1-x} \mathrm{Ti}_{x} \mathrm{O}_{3}$ revealed that the substitution of $\mathrm{Ti}^{4+}$ for $\mathrm{V}^{4+}$ ions may also transform the strong-correlation fluctuations by narrowing the $\pi^{*}$ band in addition to introducing Anderson localized states. ${ }^{120}$

Based on the transport study of STVO thin films, the mechanisms behind the observed MIT are rather complicated due to competing effects among electron correlation, disorder, and percolation. In the STVO system with $\mathrm{Ti}^{4+}\left(3 d^{0}\right)$ and $\mathrm{V}^{4+}\left(3 d^{1}\right)$ ions randomly occupying the $\mathrm{B}$ sites, the $\mathrm{Ti}^{4+}$ ions perturb the periodic potential of the $\mathrm{V}$ $3 d$ band so as to introduce significant disorder and Anderson-localized states. In addition, lattice distortions caused by epitaxial strain may also have dramatic consequences on the transport properties of the STVO thin films. As revealed by crystal structure analysis, all the STVO films had the same in-plane lattice parameter $a$ since the films were completely strained to the LSAT substrates, and the out-of-plane lattice parameter $c$ was found to increase with decreasing $x$. The $c / a$ ratio is listed in Table 6-2. The lattice structure gradually transformed from horizontally stretched to compressed as $x$ decreases, and the $x=0.67$ film was closer to a cubic lattice. The lattice distortion with decreasing $x$ causes a greater separation between the adjacent $\mathrm{V}^{4+}$ ions and thus less overlap between the $3 d$ orbital states, which may result in a narrower $\mathrm{V} 3 d$ band. In the bandwidthcontrolled MIT (BC-MIT), a MIT occurs when the $U / W$ ratio is beyond a critical value. ${ }^{4}$ 
Thus, the decrease in the vanadium concentration $x$ results in a reduction of the effective bandwidth $W$, and the films undergo a composition dependent MIT when the vanadium concentration crosses $x=0.67$.

Percolation phenomena may play a major role in the transport properties of STVO thin films. ${ }^{111,12}$ Since SVO is a correlated metal and STO is a band insulator, the solid solution STVO may exhibit spatial as well as disorder-induced inhomogeneity with both metallic and insulating phases coexisting. Such an electronic phase separation would give rise to percolation phenomena. The transport behaviors of STVO films can be described as percolative conduction through metallic regions embedded in an insulating matrix. The insulating phases are dominant in the STVO films with lower vanadium concentration, and the temperature dependence of conductivity followed Mott's variable range hopping (VRH) model. As the vanadium concentration $x$ increases, the amount of the metallic phase grows and gradually becomes increasingly dominant. When $x$ crosses the percolation threshold $(x=0.67)$, metallic paths are formed and thereby the STVO films with higher vanadium concentration exhibit metallic behaviors. The electronic structures of the STVO thin films will be further studied by soft x-ray spectroscopy, the evolution of the electronic structure across the MIT may reveal the driving force behind the MIT.

\subsection{Summary}

In this chapter, I have investigated the chemical doping effects in the vanadate thin films. High-quality epitaxial $\operatorname{SrTi}_{1-x} \mathrm{~V}_{x} \mathrm{O}_{3}(\mathrm{STVO})(0 \leq x \leq 1)$ thin films were grown on (001)-oriented LSAT substrates using the PED technique. All the films in this study 
had a thickness of $\sim 16 \mathrm{~nm}$. The transport properties of the STVO thin films were found to be strongly dependent on the film composition. A temperature driven metal-insulator transition (MIT) was observed in the $x=0.67$ film at $95 \mathrm{~K}$. The films with higher vanadium concentration $\left(x=0.83\right.$ and 1) were metallic, following the $T^{2}$ law corresponding to a Fermi liquid system. The ones with lower vanadium concentration $(x$ $=0.33$ and 0.5 ) were semiconducting, and their transport behaviors were governed by Mott's variable range hopping mechanism. The mechanisms behind the observed MIT are complicated due to the competing effects among electron correlation, disorder, and percolation. Percolation likely plays a major role in the system for which the transport properties are determined by the topology of the coexisting metallic and insulating regions. 


\section{Chapter 7 Transport in $\mathrm{SrVO}_{3} / \mathrm{SrTiO}_{3}$ Superlattices}

\subsection{Introduction}

Recent advances in the synthesis of artificial heterostructures composed of transition metal oxides with atomic precision have opened up new perspectives for the investigation of novel electronic interface phenomena. In many cases the heterointerfaces between two insulators exhibit exotic metallic behavior. For instance, two-dimensional electron gas (2DEG) and interfacial superconductivity have been discovered at the

heterointerface between two band insulators $\mathrm{LaAlO}_{3}$ (LAO) and $\mathrm{SrTiO}_{3}$ (STO). ${ }^{139,140}$ Also, the $\mathrm{LaTiO}_{3} / \mathrm{SrTiO}_{3}(\mathrm{LTO} / \mathrm{STO})$ superlattices comprised of a Mott insulator LTO and a band insulator STO have been reported to exhibit metallic conductivity similar to the bulk solid solution of $\mathrm{La}_{1-x} \mathrm{Sr}_{x} \mathrm{TiO}_{3}{ }^{141}$

In Chapter 5, two dimensional Mott insulators have been demonstrated in the vanadate ultrathin films with thicknesses of only a few nanometers, whereas thicker films remain metallic. In this chapter, an $\mathrm{SrVO}_{3}(\mathrm{SVO})$ ultrathin film of a few unit cells is embedded in Mott-insulator/band-insulator SVO/STO superlattices. Transport phenomena in the SVO/STO superlattices are investigated by varying the number of repetitions of the SVO/STO bilayers and the thickness of individual SVO and STO layers. The results are compared with single layer SVO films and $\operatorname{SrTi}_{1-x} \mathrm{~V}_{x} \mathrm{O}_{3}(\mathrm{STVO})(0 \leq x \leq$ 1) films as presented in the previous chapters, which will shed light on the insulating nature of the vanadate ultrathin films. 


\subsection{SVO/STO Superlattice Growth}

High-quality epitaxial $\mathrm{SrVO}_{3} / \mathrm{SrTiO}_{3}(\mathrm{SVO} / \mathrm{STO})$ superlattices were deposited on (001)-oriented LSAT substrates by PED. The SVO/STO superlattices were grown by sequential deposition of the constituent SVO and STO layers from two individual ceramic targets using two electron-beam guns. For the growth of the SVO layer, the electron-beam gun was operated at a potential of $12 \mathrm{kV}$ and a rate of $5 \mathrm{~Hz}$ to ablate a $\mathrm{Sr}_{2} \mathrm{~V}_{2} \mathrm{O}_{7}$ target, the growth rate for SVO was calibrated by XRR measurements and found to be $1.8 \AA / \mathrm{min}$. For the growth of the STO layer, a $10 \mathrm{kV}$ potential was applied to ablate a stoichiometric STO target at a rate of $5 \mathrm{~Hz}$ to achieve a similar growth rate as SVO. The thickness of each constituent layer in SVO/STO superlattices was controlled by varying the number of pulses for each of the two materials based on the calibrated growth rates. During the deposition, both SVO and STO layers were grown at a substrate temperature of $800{ }^{\circ} \mathrm{C}$ in a $10 \mathrm{mTorr}$ Ar atmosphere. The growth conditions are listed in Table 7-1. The SVO/STO superlattices are denoted as $\left[(\mathrm{SVO})_{m} /(\mathrm{STO})_{n}\right]_{r}$, where $m(n)$ indicates the thickness of SVO(STO) layer in unit cells, and $r$ represents the number of repetitions of the SVO/STO bilayers.

Table 7-1 Growth conditions of SVO/STO superlattices

\begin{tabular}{lcccc}
\hline & $\begin{array}{c}\text { Voltage } \\
(\mathbf{k V})\end{array}$ & $\begin{array}{c}\text { Pulse Rate } \\
(\mathbf{H z})\end{array}$ & $\begin{array}{c}\text { Ar Pressure } \\
(\mathbf{m T o r r})\end{array}$ & $\begin{array}{c}\text { Temperature } \\
\left({ }^{\circ} \mathbf{C}\right)\end{array}$ \\
\hline SVO & 12 & 5 & 10 & 800 \\
STO & 10 & 5 & 10 & 800 \\
\hline
\end{tabular}




\section{$7.3\left[(\mathrm{SVO})_{7} /(\mathrm{STO})_{4}\right]_{r}$ Superlattices}

\subsubsection{Structural Properties}

$\left[(\mathrm{SVO})_{7} /(\mathrm{STO})_{4}\right]_{r}$ superlattices with $r=1,2,3,5,7,9$ are examined in this section. The wide range out-of-plane XRD scans shown in Fig. 7-1 (a) confirmed single phase epitaxial SVO/STO superlattices grown on (001)-oriented LSAT substrates. The Kiessig fringes around the peaks indicated smooth interfaces between the SVO and STO layers. As the number of repetitions $r$ increased from 1 to 9 , the first-order satellite peaks became sharper. Fig. 7-1 (b) shows the XRD scan around the (002) symmetric reflection of the superlattice with $r=9$. The zeroth-order superlattice peak appeared near the LSAT substrate peak. The satellite peaks up to the second order were observed indicating longrange order in the superlattice. The thickness of SVO/STO bilayer $D$ can be estimated from the angular distance between adjacent satellite peaks by the following relationship $^{142}$ :

$$
D=\frac{\lambda}{2 \Delta \eta_{i} \cos \theta_{B}}
$$

where $\lambda, \Delta \eta_{i}$, and $\theta_{B}$ represents the x-ray wavelength, the angular spacing of the adjacent satellite maxima, and the Bragg reflection angle of the superlattice, respectively. The thickness $D$ was estimated to be $4.4 \mathrm{~nm}$, which was consistent with the SVO/STO bilayer consisting of 7 SVO unit cells and 4 STO unit cells. Rocking curves shown in the inset of Fig. 7-1 (b) were measured on the zeroth-order superlattice and substrate (002) peaks. The full-width at half-maximum (FWHM) values for the superlattice and substrate peaks 
were around 600 arcsec and 200 arcsec, respectively. The narrow FWHM of the superlattice is a further indication of high quality superlattice structure. The reciprocal space mapping on the (103) asymmetric reflection of the same SVO/STO superlattice with $r=9$ is shown in Fig. 7-1 (c). The in-plane lattice parameter of the superlattice matched well with the underlying LSAT substrate and hence was coherently strained with the substrate. 

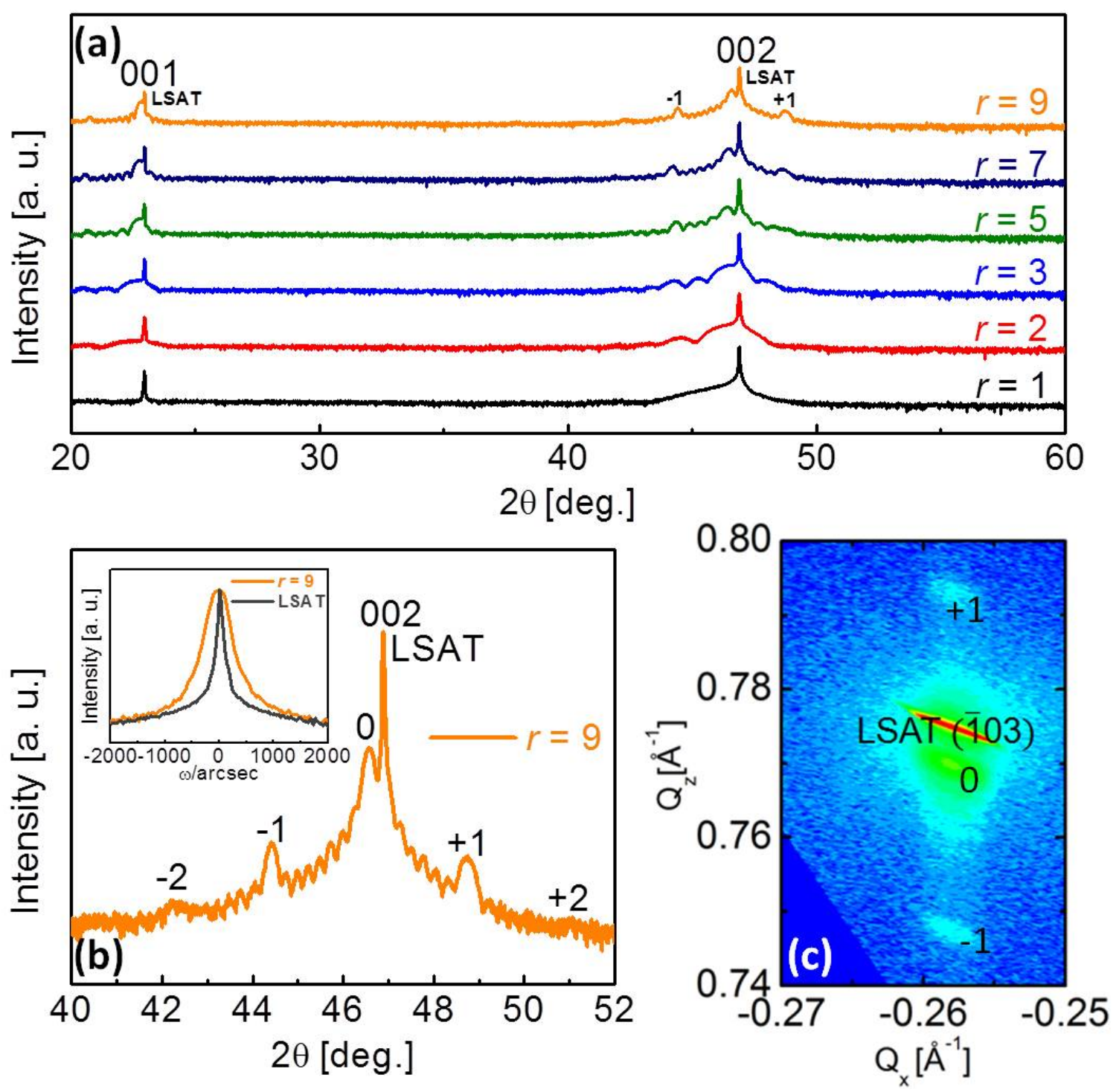

Figure 7-1 (a) Wide range out-of-plane XRD scans for the $\left[(\mathrm{SVO})_{7} /(\mathrm{STO})_{4}\right]_{r}$ superlattices with $r=1,2,3,5,7,9$ grown on (001)oriented LSAT substrates showing that no secondary phases are present. The first-order satellite peaks are noted. (b) XRD scan around the (002) reflection of SVO/STO superlattice with $r=9$ showing Kiessig fringes, and satellite peaks up to second order are noted. (Inset) Rocking curves around the (002) zeroth-order superlattice peak and the underlying LSAT substrate peak. (c) The reciprocal space mapping on (103) reflection for the same SVO/STO superlattice with $r=9$. 


\subsubsection{Transport Properties}

Fig. $7-2$ (a) shows the sheet resistance of $\left[(\mathrm{SVO})_{7} /(\mathrm{STO})_{4}\right]_{r}(1 \leq r \leq 9)$ superlattices as a function of temperature in the temperature range of 5-300 K. A systematic increase in the sheet resistance of the superlattices with decreasing $r$ was observed. All the superlattices with $r \geq 3$ exhibited metallic behaviors with sheet resistances of $\sim 10^{2} \Omega / \mathrm{sq}$. at room temperature, decreasing slowly with decreasing temperature. The observed metallic behavior indicates that the insulating STO layer with a thickness of 4 unit cells is sufficiently thin for tunneling. ${ }^{143}$ In contrast, the superlattice with $r=1$ showed semiconducting behavior with a much higher sheet resistance of $\sim 3 \times 10^{5} \Omega /$ sq. at room temperature and a rapid increase in the resistance with decreasing temperature was also observed. The superlattice with $r=2$ was found to lie at the boundary of the metallic and insulating superlattices showing a temperature-driven metalinsulator transition (MIT) at $115 \mathrm{~K}$.

Fig. 7-2 (b)-(d) show the detailed temperature dependence of the transport behavior for the superlattices. All the superlattices with $r \geq 3$ were metallic, a representative electrical resistivity as a function of temperature for the superlattice with $r$ $=9$ is shown in Fig. 7-2 (b). The resistivity followed the $\rho=\rho_{0}+A T^{2}$ relationship, characteristic of electron-electron interactions in a Fermi liquid model. A slight deviation from the $T^{2}$ dependence was observed below $100 \mathrm{~K}$. In Fig. 7-2 (c), a temperature driven MIT characterized by a resistivity upturn at a transition temperature $T_{M I T}$ of $115 \mathrm{~K}$ is shown for the superlattice with $r=2$. Above $T_{M I T}$, metallic behavior was observed with the temperature dependence of resistivity still following the $T^{2}$ dependence. As the 
temperature was lowered below $T_{M I T}$, the resistivity gradually increased. The fitting parameters of $\rho_{0}$ and $A$ for the superlattices with $r \geq 2$ are listed in Table 7-2, which are comparable to the single layer SVO films (Table 5-2). The superlattice with $r=1$ exhibited semiconducting behavior. As plotted in Fig. 7-2 (d), the temperature dependence of conductivity followed the $\exp \left[-\left(T_{0} / T\right)^{1 / 4}\right]$ law corresponding to the Mott's variable range hopping (VRH) model in the entire temperature range. The fitting parameter $T_{0}$ along with all the resistivity data are also listed in Table 7-2.

Table 7-2 Transport data of the $\left[(\mathrm{SVO})_{7} /(\mathrm{STO})_{4}\right]_{r}(1 \leq r \leq 9)$ superlattices.

\begin{tabular}{|c|c|c|c|c|c|}
\hline $\begin{array}{c}\text { Number of Repetitions, } \\
r\end{array}$ & $\begin{array}{c}\rho(300 \mathrm{~K}) \\
(\Omega \mathrm{cm})\end{array}$ & $\begin{array}{l}\rho(5 \mathrm{~K}) \\
(\Omega \mathrm{cm})\end{array}$ & $\begin{array}{c}\rho_{0} \\
(\Omega \mathrm{cm})\end{array}$ & $\begin{array}{c}A \\
\left(\Omega \mathrm{cm} / \mathrm{K}^{2}\right)\end{array}$ & $\begin{array}{c}T_{0} \\
(\mathbf{K})\end{array}$ \\
\hline 9 & $2.73 \times 10^{-4}$ & $1.94 \times 10^{-4}$ & $1.87 \times 10^{-4}$ & $9.57 \times 10^{-10}$ & - \\
\hline 7 & $3.10 \times 10^{-4}$ & $2.31 \times 10^{-4}$ & $2.19 \times 10^{-4}$ & $1.00 \times 10^{-9}$ & - \\
\hline 5 & $3.22 \times 10^{-4}$ & $2.29 \times 10^{-4}$ & $2.20 \times 10^{-4}$ & $1.12 \times 10^{-9}$ & - \\
\hline 3 & $3.09 \times 10^{-4}$ & $2.12 \times 10^{-4}$ & $2.09 \times 10^{-4}$ & $1.11 \times 10^{-9}$ & - \\
\hline 2 & $4.35 \times 10^{-4}$ & $4.00 \times 10^{-4}$ & $3.55 \times 10^{-4}$ & $8.92 \times 10^{-10}$ & - \\
\hline 1 & 0.14 & $>10$ & - & - & $8.12 \times 10^{6}$ \\
\hline
\end{tabular}



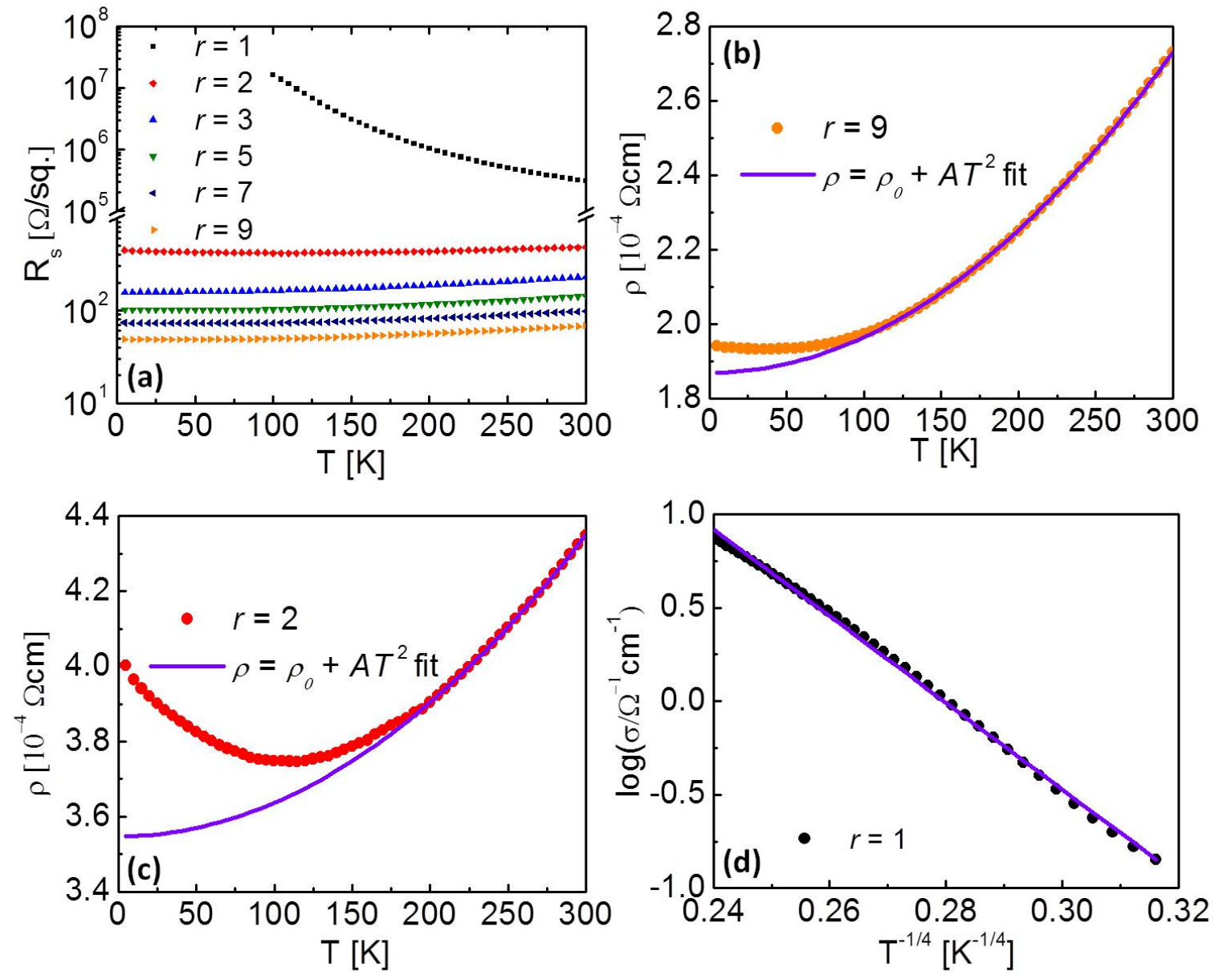

Figure 7-2 (a) Sheet resistance as a function of temperature for the $\left[(\mathrm{SVO})_{7} /(\mathrm{STO})_{4}\right]_{r}$ superlattices with $r=1,2,3,5,7,9$. Temperature dependence of electrical resistivity for the same set of SVO/STO superlattices with (b) $r=9$, (c) $r=2, \rho=\rho_{0}+A T^{2}$ fits are also shown. (d) Logarithm of conductivity as a function of $T^{-1 / 4}$ with a linear fit to the data for the SVO/STO bilayer $(r=1)$.

As shown in Table 7-3, the transport behaviors in the SVO/STO superlattices with different number of repetitions $r$ are summarized in terms of the total thickness of SVO layers. The results agree very well with the thickness-dependent transport behaviors observed in single layer SVO films. In Chapter 5, it has been demonstrated that single 
layer SVO films with thicknesses less than $6.5 \mathrm{~nm}$ showed a temperature-driven MIT, whereas thicker films were metallic. Furthermore, the transition temperature $T_{M I T}$ of the $5.7 \mathrm{~nm}$ SVO film was found to be $120 \mathrm{~K}$, which is quite close to the $T_{M I T}$ observed in the SVO/STO superlattice $(r=2)$ with a similar total thickness of SVO layers. Such a good agreement may imply that there is coupling between the SVO layers by tunneling through the STO layers.

Table 7-3 Transport behaviors in the $\left[(\mathrm{SVO})_{7} /(\mathrm{STO})_{4}\right]_{r}(1 \leq r \leq 9)$ superlattices in terms of total thickness of SVO layers.

\begin{tabular}{ccc}
\hline Number of Repetitions, $\boldsymbol{r}$ & Total Thickness of SVO layers & Transport Behavior \\
\hline $\mathbf{9}$ & $24.3 \mathrm{~nm}(63$ u.c. $)$ & Metallic \\
\hline $\mathbf{7}$ & $18.9 \mathrm{~nm}(49$ u.c. $)$ & Metallic \\
$\mathbf{5}$ & $13.5 \mathrm{~nm}(35$ u.c. $)$ & Metallic \\
$\mathbf{3}$ & $8.1 \mathrm{~nm}(21$ u.c. $)$ & Metallic \\
$\mathbf{2}$ & $5.4 \mathrm{~nm}(14$ u.c. $)$ & MIT at $115 \mathrm{~K}$ \\
$\mathbf{1}$ & $2.7 \mathrm{~nm}(7$ u.c. $)$ & Semiconducting \\
\hline
\end{tabular}

Magnetoresistance (MR) measurements below $20 \mathrm{~K}$ in a perpendicular magnetic field were performed to understand the transport mechanism in the SVO/STO superlattices. As shown in Fig. 7-3 (a) and (b), a small positive MR value was observed for both of the superlattices with $r=9$ and $r=2$ for all the temperatures. Furthermore, an increase in the MR was observed with decreasing temperature. At $5 \mathrm{~K}$, the MR was less than $0.3 \%$ at a high field of $7 \mathrm{~T}$, which was quite similar to the single layer SVO film as 
shown in Fig. 5-3 (a). In addition, the MR was found to be roughly proportional to $B^{2}$ at each temperature point as observed in the single layer SVO film. The positive MR is an indication of electron-electron interactions, and thereby the observed MIT induced in the superlattice with $r=2$ may not be dominated by the disorder-induced localization, since the localization effect leads to a negative MR. ${ }^{114,115,116,118}$
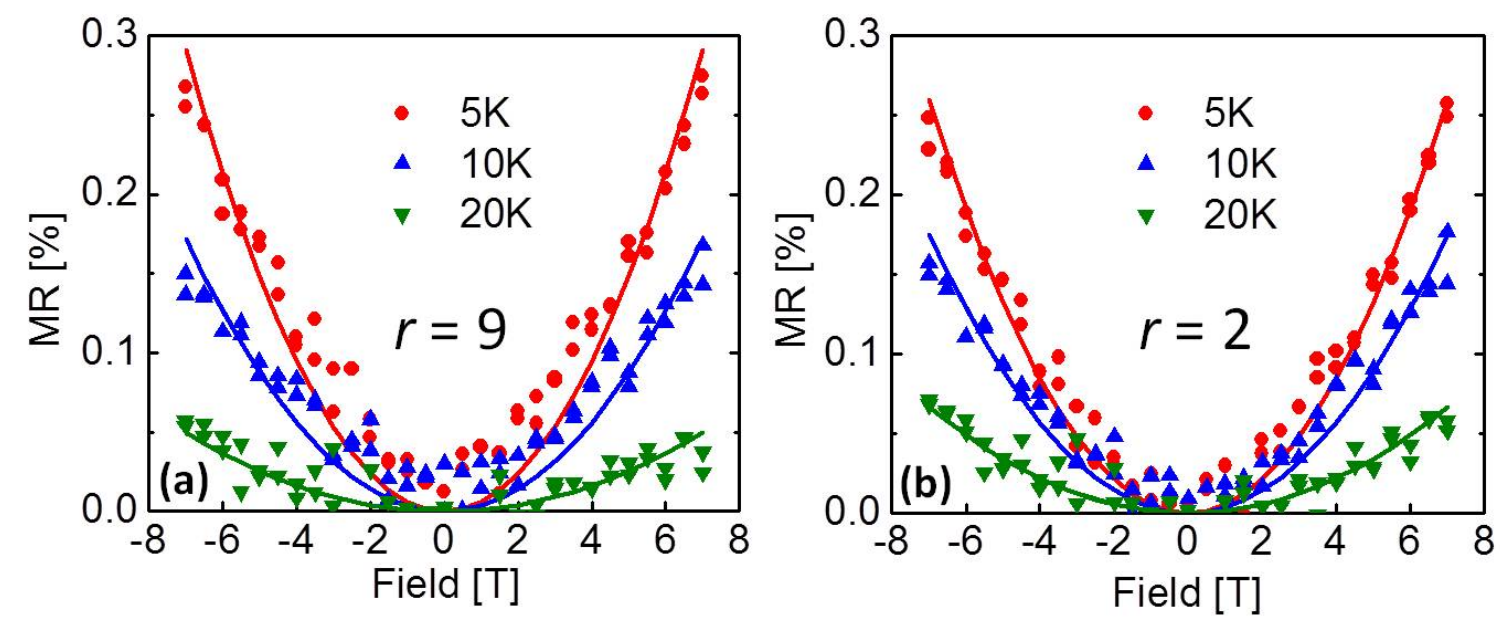

Figure 7-3 Normalized out-of-plane $\mathrm{MR}$ of the $\left[(\mathrm{SVO})_{7} /(\mathrm{STO})_{4}\right]_{r}$ superlattices with (a) $r=9$ and (b) $r=2$ between $5 \mathrm{~K}$ and $20 \mathrm{~K}$ with the field up to $7 \mathrm{~T}$. The parabolic fits at each temperature are also shown in lines.

\subsubsection{MIT Mechanism}

The electrical transport behavior in the SVO/STO superlattices can be regarded as conduction through parallel-coupled SVO layers. Therefore the inverse of the sheet resistance of the superlattices $1 / R_{S}$ should be proportional to the number of repetitions $r$. In Fig. 7-4 (a), 1/R $R_{S}$ of the superlattices with $2 \leq r \leq 9$ are plotted as a function of $r$ at three different temperatures. A linear relationship between $1 / R_{S}$ and $r$ was observed at 
each temperature point as expected. It has been demonstrated that the sheet resistance of the superlattice $R_{S}$ in the $\mathrm{LaNiO}_{3} / \mathrm{SrTiO}_{3}(\mathrm{LNO} / \mathrm{STO})$ superlattices can be modeled as follows: $:^{144}$

$$
\frac{1}{R_{S}}=\frac{1}{R_{I}}(2 r-2)+\frac{1}{R_{L N O}} r
$$

where $R_{L N O}$ and $R_{I}$ represent the sheet resistance of the individual LNO layer and the interface, respectively. The $(2 r-2)$ interfaces can be understood assuming that only interfaces that are in contact with LNO layers from both sides are conductive. Using the slopes and intercepts of the linear fits to the $1 / R_{S}$ vs. $r$ plots as modeled in Equation 7.2, the sheet resistance of the individual SVO layer embedded in the SVO/STO superlattice with $2 \leq r \leq 9$ can be determined. Fig. 7-4 (b) shows the calculated sheet resistance of the SVO layer in superlattices in the temperature range of $100-300 \mathrm{~K}$. The sheet resistance was $\sim 2 \times 10^{3} \Omega / \mathrm{sq}$. at room temperature and increased slightly with decreasing temperature. The measured sheet resistance of the SVO/STO bilayer $(r=1)$ is also shown in Fig. 7-4 (b) for comparison, which is 2-4 orders of magnitude higher than the calculated values. This indicates that the SVO layer embedded in the superlattices with 2 $\leq r \leq 9$ is more conductive than the single $\mathrm{SVO}$ layer. 

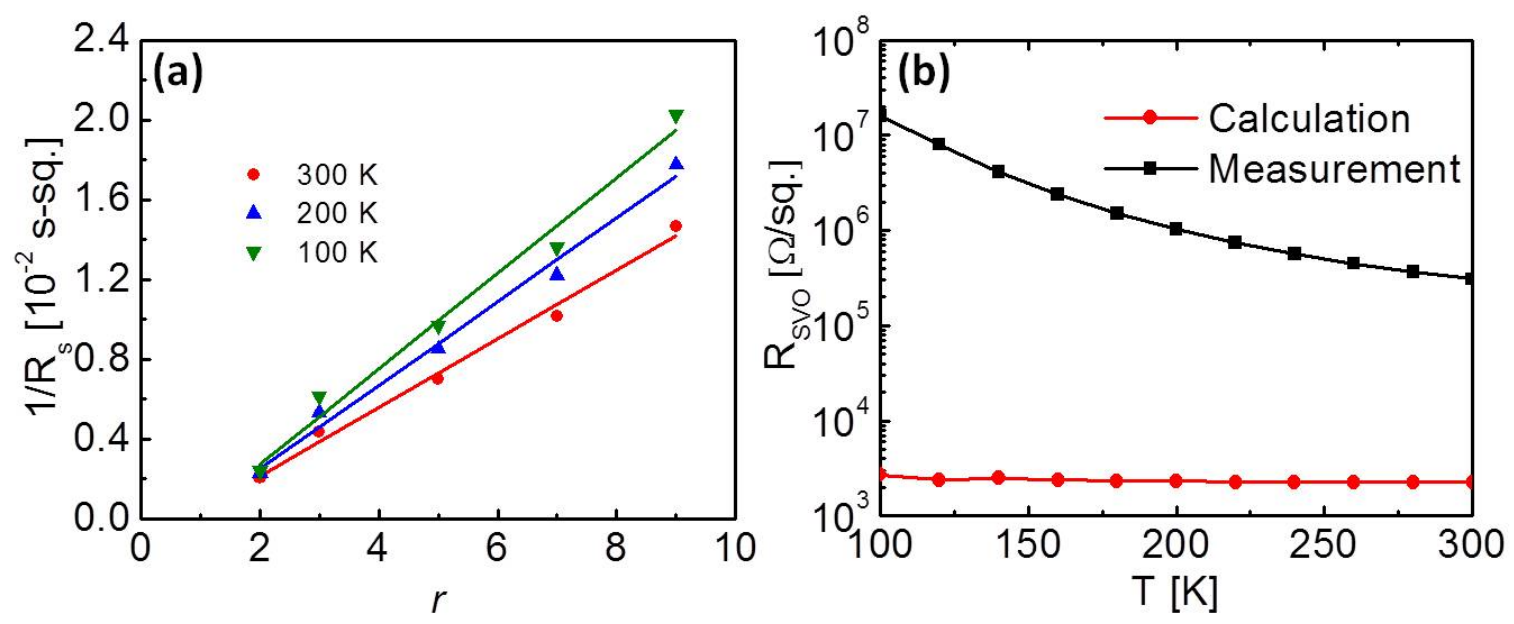

Figure 7-4 (a) The inverse of the sheet resistance of the SVO/STO superlattices as a function of the number of repetitions measured at three different temperatures. The linear fits to the data are also shown. (b) Calculated sheet resistance of the individual SVO layer embedded in the superlattices with $2 \leq r \leq 9$ and measured sheet resistance of the SVO/STO bilayer $(r=1)$.

The conductivity enhancement observed in the SVO layer embedded in the $\mathrm{SVO} / \mathrm{STO}$ superlattices is a further indication of percolation phenomena in the vanadate ultrathin films as described in Chapter 5. In the superlattices, the ultrathin SVO layer with the thickness of 7 unit cells may experience spatial inhomogeneity with both metallic and insulating phases coexisting. The insulating behavior observed in the single SVO layer can be considered as the metallic phase content just below the percolation threshold for metallic conduction. By stacking several SVO layers into the SVO/STO superlattice, the coupling between the SVO layers will increase the number of conduction pathways, resulting in the great enhancement in the conductivity in the SVO layer. ${ }^{144}$ Furthermore, as more SVO layers were added to the superlattice, the conduction pathways were formed 
beyond the percolation threshold and metallic behaviors were induced in the superlattices with $r \geq 3$.

\section{$7.4\left[(\mathrm{SVO})_{m} /(\mathrm{STO})_{n}\right]_{9}$ Superlattices}

\subsubsection{Structural Properties}

$\left[(\mathrm{SVO})_{m} /(\mathrm{STO})_{n}\right]_{9}$ superlattices with different thicknesses for the individual SVO and STO layers are investigated in this section. Single phase epitaxial SVO/STO superlattices were confirmed from wide range out-of-plane XRD scans as shown in Fig. 7-5 (a). The Kiessig fringes indicated sharp SVO/STO interfaces within the superlattices. From the angular positions of the first-order satellite peaks, the thickness of SVO/STO bilayer for each superlattice can be deduced using Equation 7.1, and all the superlattice structures were confirmed to be as designed. Furthermore, since SVO has a smaller lattice parameter than STO, the zeroth-order superlattice peak progressively shifted to the higher $2 \theta$ value with the increasing thickness ratio $m / n$. Based on the peak positions, the average out-of-plane lattice parameters of the superlattices were determined and plotted as a function of $m /(m+n)$ in Fig. 7-5 (b). A nearly linear relationship was observed in the average lattice parameter and the superlattice composition corresponding to the Vegard's law, which is quite similar to the $\operatorname{SrTi}_{1-x} \mathrm{~V}_{x} \mathrm{O}_{3}$ thin films as described in Chapter 6 . 

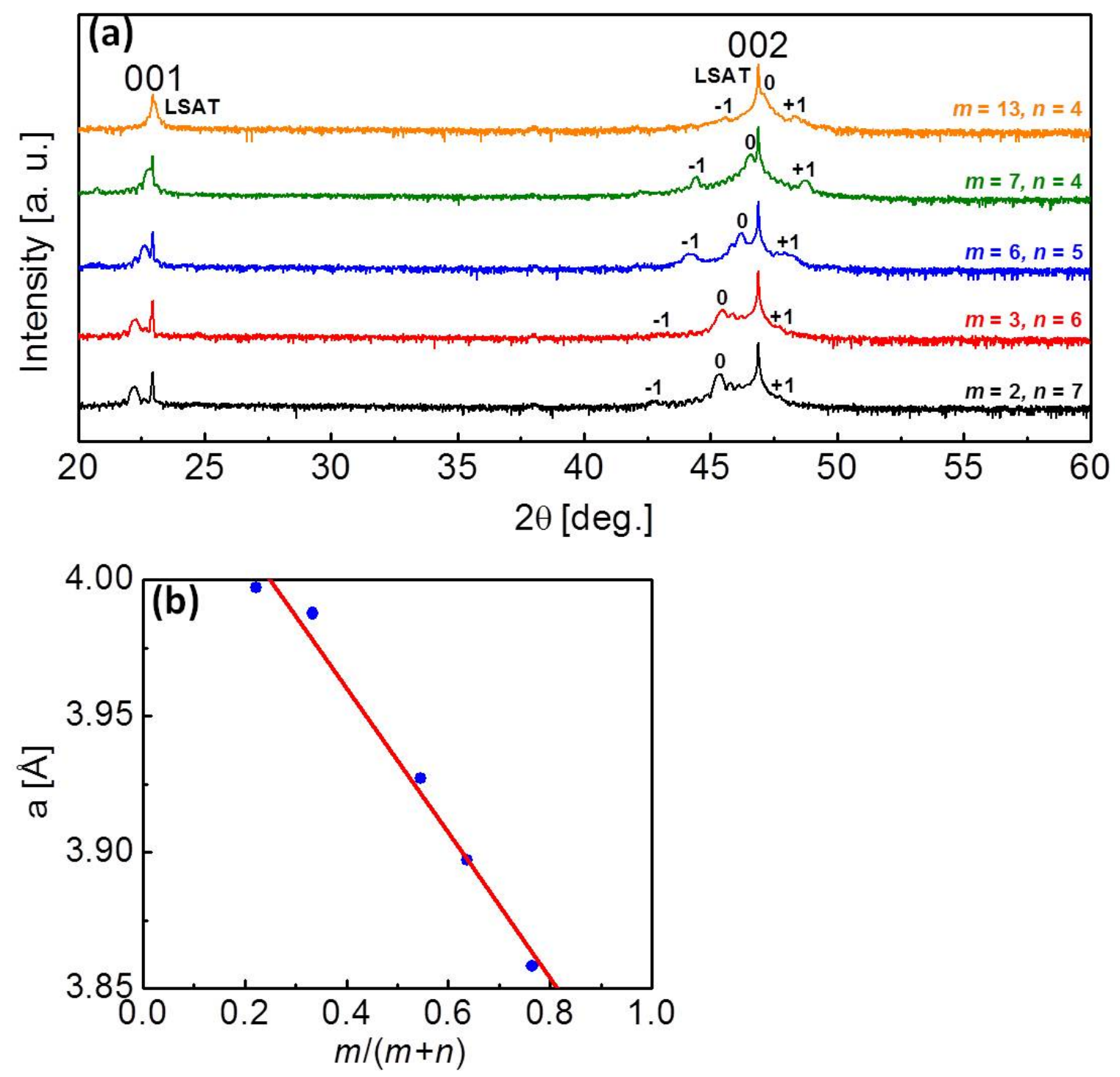

Figure 7-5 (a) Wide range out-of-plane XRD scans for the $\left[(\mathrm{SVO})_{m} /(\mathrm{STO})_{n}\right]_{9}$ superlattices grown on $(001)$-oriented LSAT substrates showing that no secondary phases are present and the first-order satellite peaks are noted. (b) Calculated average out-of-plane lattice parameter of the superlattices as a function of $m /(m+n)$. A linear fit is also shown. 


\subsubsection{Transport Properties}

The temperature dependence of the sheet resistance of the $\left[(\mathrm{SVO})_{m} /(\mathrm{STO})_{n}\right]_{9}$ superlattices were measured in the temperature range of 5-300 K as shown in Fig. 7-6 (a). In the entire temperature range, the sheet resistance systematically increased with decreasing $m / n$ ratio. Moreover, different transport behaviors were observed in the superlattices with different $m$ and $n$. The superlattice with $m=6$ and $n=5$ was found to exhibit a temperature-driven MIT at $235 \mathrm{~K}$. The superlattices with thicker SVO layers and thinner STO layers ( $m=13, n=4$ and $m=7, n=4)$ were metallic, on the other hand, the ones with thinner SVO layers and thicker STO layers $(m=3, n=6$ and $m=2, n=7)$ were semiconducting.

The detailed transport behaviors of the superlattices in the three regions are shown in Fig. 7-6 (b)-(d). The superlattice with $m=13$ and $n=4$ exhibited metallic behavior (Fig. 7-6 (b)). Over the entire temperature range of 5-300 K, the temperature dependence of electrical resistivity followed the $\rho=\rho_{0}+A T^{2}$ relationship corresponding to the Fermi liquid model. As listed in Table 7-4, the fitting parameters $\rho_{0}$ and $A$ were quite close to the values obtained from the single layer SVO films as presented in Chapter 4. Fig. 7-6 (c) shows the temperature-driven MIT in the superlattice with $m=6$ and $n=5$ characterized by a resistivity upturn at a transition temperature of $235 \mathrm{~K}$. The superlattice with $m=2$ and $n=7$ exhibited semiconducting behavior as plotted in Fig. 7-6 (d). In the entire temperature range of $5-300 \mathrm{~K}$, the conductivity followed the $\exp \left[-\left(T_{0} / T\right)^{1 / 2}\right]$ dependence corresponding to the Efros-Shklovskii variable range hopping (ES-VRH) 
model, in which electron-electron interactions open up a coulomb gap at the Fermi level. ${ }^{145}$ The transport data along with all the fitting parameters are listed in Table 7-4.
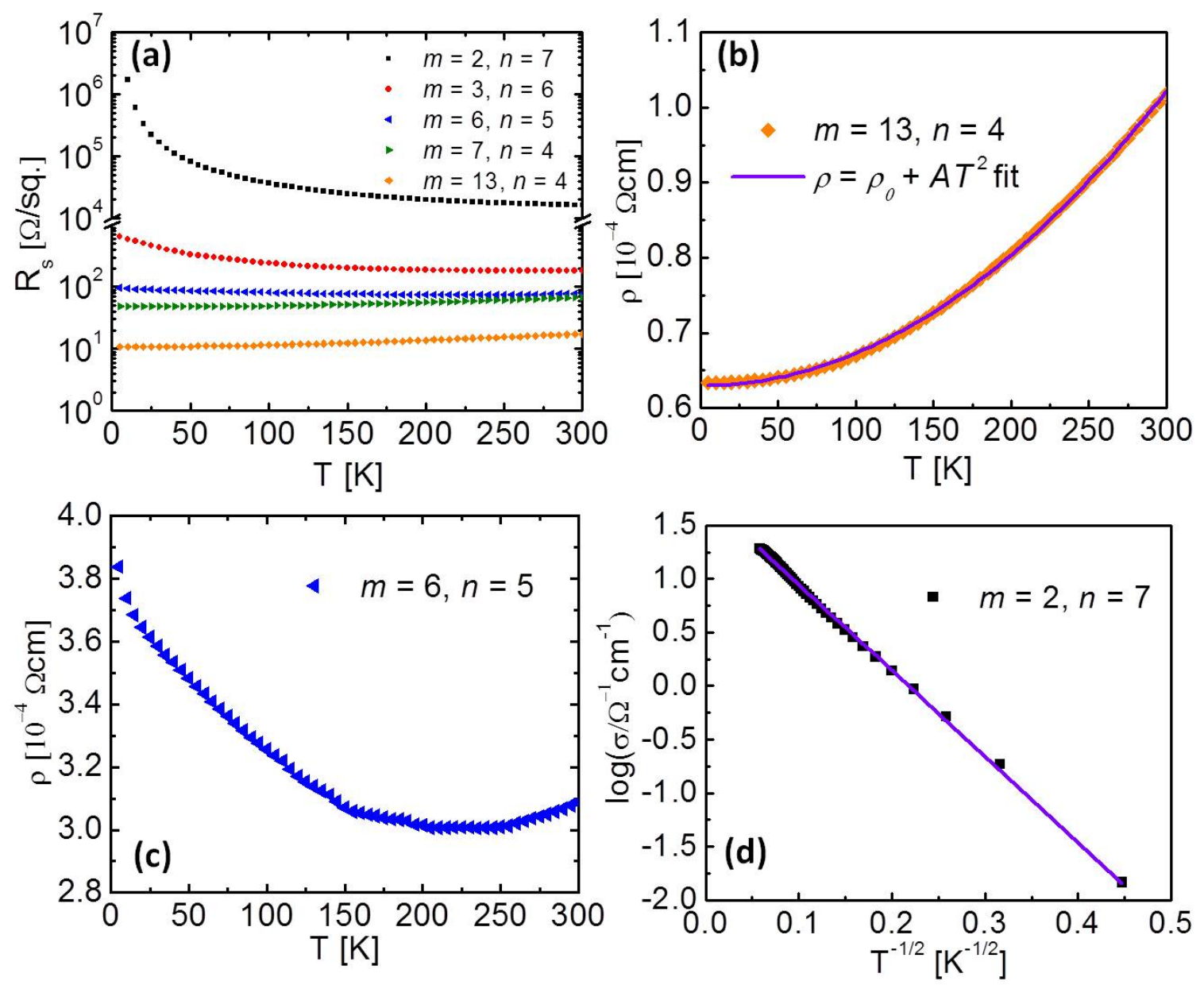

Figure 7-6 (a) Sheet resistance as a function of temperature for the $\left[(\mathrm{SVO})_{m} /(\mathrm{STO})_{n}\right]_{9}$ superlattices. Temperature dependence of electrical resistivity for the same set of SVO/STO superlattices with (b) $m=13, n=$ 4, $\rho=\rho_{0}+A T^{2}$ fit is also shown, and (c) $m=6, n=5$. (d) Logarithm of conductivity as a function of $T^{1 / 2}$ for the superlattice with $m=2, n=7$. A linear fit to the data is also shown. 
Table 7-4 Transport data of the $\left[(\mathrm{SVO})_{m} /(\mathrm{STO})_{n}\right]_{9}$ superlattices.

\begin{tabular}{|c|c|c|c|c|c|c|c|}
\hline$m$ & $n$ & $m /(m+n)$ & $\begin{array}{c}\rho(300 \mathrm{~K}) \\
(\Omega \mathrm{cm})\end{array}$ & $\begin{array}{l}\rho(5 \mathrm{~K}) \\
(\Omega \mathrm{cm})\end{array}$ & $\begin{array}{c}\rho_{0} \\
(\Omega \mathrm{cm})\end{array}$ & $\begin{array}{c}A \\
\left(\Omega \mathrm{cm} / \mathbf{K}^{2}\right)\end{array}$ & $\begin{array}{r}T_{0} \\
(\mathbf{K})\end{array}$ \\
\hline 13 & 4 & 0.76 & $1.02 \times 10^{-4}$ & $6.34 \times 10^{-5}$ & $6.29 \times 10^{-5}$ & $4.37 \times 10^{-10}$ & - \\
\hline 7 & 4 & 0.64 & $2.73 \times 10^{-4}$ & $1.94 \times 10^{-4}$ & $1.87 \times 10^{-4}$ & $9.57 \times 10^{-10}$ & - \\
\hline 6 & 5 & 0.55 & $3.09 \times 10^{-4}$ & $3.84 \times 10^{-4}$ & - & - & - \\
\hline 3 & 6 & 0.33 & $5.84 \times 10^{-4}$ & $2.07 \times 10^{-3}$ & - & - & - \\
\hline 2 & 7 & 0.22 & 0.05 & 68.2 & - & - & $3.42 \times 10^{2}$ \\
\hline
\end{tabular}

\subsubsection{MIT Mechanism}

The transport behaviors observed in the $\left[(\mathrm{SVO})_{m} /(\mathrm{STO})_{n}\right]_{9}$ superlattices can also be explained as percolative conduction through the coupled SVO layers. The reduction in the STO layer thickness would cause an increase in the interlayer electron hopping through the STO layer, creating more conduction pathways. ${ }^{146,147}$ As the conduction pathways were formed beyond the percolation threshold, the superlattices exhibited metallic behaviors. Furthermore, the vanadium concentration in the superlattices can be determined from $m /(m+n)$ as listed in Table 7-4. The superlattice that undergoes a temperature-driven MIT at $235 \mathrm{~K}$ has a vanadium concentration of 0.55 which can be considered as very close to the percolation threshold. The superlattices with higher vanadium concentration were metallic and the ones with lower vanadium concentration were semiconducting. Such transport behaviors in the $\left[(\mathrm{SVO})_{m} /(\mathrm{STO})_{n}\right]_{9}$ superlattices were quite similar to the $\operatorname{SrTi}_{1-x} \mathrm{~V}_{x} \mathrm{O}_{3}(\mathrm{STVO})$ thin films as discussed in Chapter 6. In the 
STVO films, the percolation threshold was found to be near $x=0.67$, which was slightly larger than that observed in the SVO/STO superlattice. This can be understood by considering SVO/STO superlattices as STVO solid solution thin films with dopants

confined in single layers. ${ }^{148}$ Therefore, the interlayer coupling in the highly ordered superlattices may form more conduction pathways than in the single layer STVO films with randomly distributed metallic and insulating phases, leading to a lower percolation threshold in the SVO/STO superlattice. Moreover, the SVO layer might be strained by the adjacent STO layers, and the magnitude of the strain is determined by the thickness ratio between the SVO and STO layers, which may also play a role in the transport properties of the superlattices. The microstructure and interface characteristics of the SVO/STO superlattices will be further studied by cross-section transmission electron microscopy (TEM).

\subsection{Summary}

This chapter demonstrates the ability of PED to grow high quality epitaxial $\mathrm{SrVO}_{3} / \mathrm{SrTiO}_{3}(\mathrm{SVO} / \mathrm{STO})$ superlattices and to modify their transport properties by varying the number of repetitions of the SVO/STO bilayers and the thickness of individual SVO and STO layers.

The transport study of the $\left[(\mathrm{SVO})_{7} /(\mathrm{STO})_{4}\right]_{r}(1 \leq r \leq 9)$ superlattices have shown that all the superlattices with $r \geq 3$ were metallic, whereas a single SVO/STO bilayer was semiconducting. A temperature-driven MIT was observed at $115 \mathrm{~K}$ in the superlattice with $r=2$. The transport behaviors in the SVO/STO superlattice can be 
interpreted as percolation phenomena, which is evidence of electronic phase separation in the vanadate ultrathin layers as discussed in Chapter 5. As the number of repetitions $r$ increased, more conduction pathways were created by the coupling between SVO layers, resulting in the great enhancement in the conductivity in the SVO layer, as well as a crossover from insulating to metallic behavior.

$\left[(\mathrm{SVO})_{m} /(\mathrm{STO})_{n}\right]_{9}$ superlattices have also been investigated. A temperature-driven MIT was observed at $235 \mathrm{~K}$ in the superlattice with $m=6$ and $n=5$. The superlattices with thicker SVO layers and thinner STO layers $(m=13, n=4$ and $m=7, n=4)$ exhibited metallic behaviors, and the ones with thinner SVO layers and thicker STO layers ( $m=3, n=6$ and $m=2, n=7)$ were semiconducting. The observed transport behaviors also suggest that percolation plays an important role in the SVO/STO superlattices. An increase in the SVO layer thickness $m$ along with a reduction in the STO layer thickness $n$ would cause an increase in the interlayer electron hopping through the STO layer. As the conduction pathways were formed beyond the percolation threshold, metallic behaviors were observed in the superlattices with thicker SVO layers and thinner STO layers. 


\section{Chapter 8 Summary and Future Work}

\subsection{Summary}

This dissertation is an experimental study exploring the transport phenomena in the perovskite vanadate thin films. High quality epitaxial vanadate thin films were successfully synthesized using a novel pulsed electron-beam deposition (PED) tool. Methods to systematically control the metal-insulator transition (MIT) in these materials have been demonstrated, including dimensional confinement in the quantum well, chemical doping, and superlattice structures. The key findings are summarized below.

\section{Vanadate Ultrathin Films}

The transport properties of $\mathrm{SrVO}_{3}(\mathrm{SVO})$ and $\mathrm{CaVO}_{3}(\mathrm{CVO})$ thin films have been studied as a function of film thickness. For the vanadate films with thicknesses over 20 $\mathrm{nm}$, the transport properties were quite similar to the bulk. These films exhibited a metallic behavior following the $T^{2}$ law that is consistent with the Fermi liquid model. Two-dimensional Mott insulating states were demonstrated in the vanadate ultrathin films by reducing the thicknesses to only a few nanometers. The SVO films with thicknesses less than $6.5 \mathrm{~nm}$ showed a temperature-driven MIT. The transition temperature $T_{M I T}$ increased with decreasing film thickness. At near room temperature, an insulating SVO film was predicted to have a thickness of 2-3 ML, which is consistent with the electronic structure studied by photoemission spectroscopy (PES) ${ }^{70}$ The magneto-transport measurements of the SVO ultrathin films were performed at the temperature below $T_{M I T}$, 
which confirmed that the dominant driving force behind the observed MIT is electronelectron interactions other than disorder-induced localization. Similarly, a temperaturedriven MIT has also been observed in CVO films with thicknesses below $4 \mathrm{~nm}$. X-ray absorption spectroscopy (XAS) measurements showed different electronic structures in thick $(\sim 60 \mathrm{~nm})$ and ultrathin $(2-4 \mathrm{~nm})$ films of CVO. The ultrathin films were more twodimensional with the $\mathrm{V}$ charge state closer to $\mathrm{V}^{4+}$. The MIT induced in the vanadate ultrathin films may result from the dimensional crossover from a three-dimensional metal to a two-dimensional Mott insulator. As the film thickness is reduced, the atomic coordination at the interface and surface decreases, resulting in a reduction in the effective bandwidth that eventually opens a bandgap at the Fermi level. These results are the first transport study to show this crossover and confirm earlier PES results that are not nearly as conclusive.

\section{$\operatorname{SrTi}_{1-x} V_{x} \mathrm{O}_{3}$ Thin Films}

$\mathrm{SrTi}_{1-x} \mathrm{~V}_{x} \mathrm{O}_{3}(\mathrm{STVO})(0 \leq x \leq 1)$ thin films have been investigated to study the chemical doping effects. The transport properties of the STVO thin films showed a temperature-driven MIT in the $x=0.67$ film at $95 \mathrm{~K}$. The films with higher vanadium concentration ( $x=0.83$ and 1$)$ were metallic with the resistivity following the $T^{2}$ dependence. The films with lower vanadium concentration $(x=0.33$ and 0.5$)$ exhibited a semiconducting behavior that can be described by Mott's variable range hopping model. This work represents the first study of STVO thin films, and shows that effects of electron correlation, disorder, and percolation might be simultaneously present in the system. Percolation seems to be the major factor that drives the MIT, for which the 
transport properties of the STVO thin films can be determined by the topology of the coexisting metallic and insulating regions.

\section{$\mathrm{SrVO}_{3} / \mathrm{SrTiO}_{3} \mathrm{Superlattices}$}

The transport properties of $\mathrm{SrVO}_{3} / \mathrm{SrTiO}_{3}$ (SVO/STO) superlattices have been investigated by varying the number of repetitions of the SVO/STO bilayers and the thickness of individual SVO and STO layers. For the $\left[(\mathrm{SVO})_{7} /(\mathrm{STO})_{4}\right]_{r}(1 \leq r \leq 9)$ superlattices, a temperature-driven MIT was observed at $115 \mathrm{~K}$ in the superlattice with $r$ =2. All the superlattices with $r \geq 3$ were metallic, whereas a single SVO/STO bilayer was semiconducting. The transport behaviors in the SVO/STO superlattices can be described as percolation phenomena, which further indicate the coexisting metallic and insulating regions in the SVO ultrathin layers. Therefore, the coupling between SVO layers embedded in the superlattice would create more conduction pathways with increasing $r$, resulting in a crossover from insulating to metallic behavior. For the $\left[(\mathrm{SVO})_{m} /(\mathrm{STO})_{n}\right]_{9}$ superlattices, the one with $m=6$ and $n=5$ showed a temperaturedriven MIT at $235 \mathrm{~K}$. The superlattices with thinner SVO layers and thicker STO layers ( $m=3, n=6$ and $m=2, n=7$ ) were semiconducting, whereas the ones with thicker SVO layers and thinner STO layers $(m=13, n=4$ and $m=7, n=4)$ were metallic. Such an increase in the SVO layer thickness $m$ along with a reduction in the STO layer thickness $n$ may cause an increase the interlayer electron hopping, creating more conduction pathways and resulting in a metallic behavior. 


\subsection{Future Work}

\section{Exploration of Rare-Earth Vanadate Thin Films}

Bulk rare-earth vanadates $\mathrm{RVO}_{3}$ are prototypical Mott insulators with a $3 d^{2}$ electronic configuration for vanadium. ${ }^{4} \mathrm{RVO}_{3}$ has an orthorhombically distorted $\mathrm{GdFeO}_{3}$-type structure at room temperature, and undergoes a structural phase transition from orthorhombic to monoclinic at $120-150 \mathrm{~K}^{4}{ }^{4} \mathrm{RVO}_{3}$ exhibits strong spin-orbital coupling. The spin and orbital orderings are strongly influenced by the lattice distortions, giving rise to various intriguing physical properties. ${ }^{149}$ Due to the great challenges in the synthesis, $\mathrm{RVO}_{3}$ thin films have rarely been investigated. In this dissertation, the ability of pulsed electron-beam deposition (PED) technique to produce high quality epitaxial $\mathrm{CaVO}_{3}(\mathrm{CVO})$ and $\mathrm{SrVO}_{3}(\mathrm{SVO})$ thin films has been demonstrated, offering great possibilities for exploring epitaxial growth of $\mathrm{RVO}_{3}$ thin films. Study of $\mathrm{RVO}_{3}$ thin films will provide new routes to modify the interplay between spin and orbital orders coupled to the lattice. Tailoring the lattice structure of $\mathrm{RVO}_{3}$ thin films by epitaxial strain or orbital tuning layers may lead to novel transport phenomena that are not obtainable in the bulk $\mathrm{RVO}_{3}$.

The successful growth of high quality epitaxial $\mathrm{RVO}_{3}$ thin films allows further investigations of A-site doping of vanadate thin films. Bandfilling-controlled metalinsulator transition (MIT) can be achieved via substitution of divalent cations $\left(\mathrm{Ca}^{2+}, \mathrm{Sr}^{2+}\right)$ on the rare-earth site. ${ }^{4}$ For example, the $\mathrm{La}_{1-x} \mathrm{Sr}_{x} \mathrm{VO}_{3}$ (LSVO) solid solution has been extensively studied in bulk. It has a mixed $\mathrm{V}^{3+}$ and $\mathrm{V}^{4+}$ valence state and shows a 
bandfilling-controlled MIT at $x \approx 0.2 .^{67,68,69}$ Recently, epitaxial $\mathrm{LaVO}_{3}$ (LVO) thin films have been successfully synthesized by pulsed laser deposition. The LVO films showed a semiconducting behavior with the resistivity close to bulk LVO. ${ }^{150,151}$ The PED technique can be employed to explore the epitaxial growth of LSVO thin films, as well as LVO/SVO superlattices. Study of the transport properties of LSVO thin films and LVO/SVO superlattices may provide new perspectives in understanding the bandfillingcontrolled MIT. 


\section{References:}

1. C. A. Mack, Fifty Years of Moore's Law. Ieee T Semiconduct M 24, 202 (2011)

2. J. Mannhart and D. G. Schlom, Oxide Interfaces-An Opportunity for Electronics. Science 327, 1607 (2010)

3. S. B. Ogale, Thin Films and Heterostructures for Oxide Electronics (Multifunctional Thin Film Series). (Springer Science \& Business Media, USA, 2006).

4. M. Imada, A. Fujimori, and Y. Tokura, Metal-Insulator Transitions. Rev Mod Phys 70, 1039 (1998)

5. N. F. Mott, The Basis of the Electron Theory of Metals, with Special Reference to the Transition Metals. Proc. Phys. Soc. London, Sect. A 62, 416 (1949)

6. N. F. Mott, Metal-Insulator Transitions, 2nd ed. (Taylor and Francis, London, 1990).

7. R. E. Peierls, Quantum Theory of Solids. (Oxford University Press, New York, 1955).

8. P. W. Anderson, Absence of Diffusion in Certain Random Lattices. Phys. Rev. 109, 1492 (1958)

9. Z. Yang, C. Y. Ko, and S. Ramanathan, Oxide Electronics Utilizing Ultrafast Metal-Insulator Transitions. Annu Rev Mater Res 41, 337 (2011)

10. M. M. Qazilbash, M. Brehm, B. G. Chae, P. C. Ho, G. O. Andreev, B. J. Kim, S. J. Yun, A. V. Balatsky, M. B. Maple, F. Keilmann, H. T. Kim, and D. N. Basov, Mott Transition in $\mathrm{VO}_{2}$ Revealed by Infrared Spectroscopy and Nano-Imaging. Science 318, 1750 (2007)

11. Y. Tokura, Correlated-Electron Physics in Transition-Metal Oxides. Phys Today 56, 50 (2003)

12. A. Asamitsu, Y. Tomioka, H. Kuwahara, and Y. Tokura, Current Switching of Resistive States in Magnetoresistive Manganites. Nature 388, 50 (1997)

13. K. Miyano, T. Tanaka, Y. Tomioka, and Y. Tokura, Photoinduced Insulator-toMetal Transition in a Perovskite Manganite. Phys Rev Lett 78, 4257 (1997)

14. M. Fiebig, K. Miyano, Y. Tomioka, and Y. Tokura, Visualization of the Local Insulator-Metal Transition in $\mathrm{Pr}_{0.7} \mathrm{Ca}_{0.3} \mathrm{MnO}_{3}$. Science 280, 1925 (1998)

15. J. Cao, E. Ertekin, V. Srinivasan, W. Fan, S. Huang, H. Zheng, J. W. L. Yim, D. R. Khanal, D. F. Ogletree, J. C. Grossmanan, and J. Wu, Strain Engineering and One-Dimensional Organization of Metal-Insulator Domains in Single-Crystal Vanadium Dioxide Beams. Nat Nanotechnol 4, 732 (2009)

16. G. Stefanovich, A. Pergament, and D. Stefanovich, Electrical Switching and Mott Transition in $\mathrm{VO}_{2}$. J Phys-Condens Mat 12, 8837 (2000)

17. T. Driscoll, H. T. Kim, B. G. Chae, M. Di Ventra, and D. N. Basov, PhaseTransition Driven Memristive System. Appl Phys Lett 95, 043503 (2009)

18. F. Cilento, C. Giannetti, G. Ferrini, S. D. Conte, T. Sala, G. Coslovich, M. Rini, A. Cavalleri, and F. Parmigiani, Ultrafast Insulator-to-Metal Phase Transition as a 
Switch to Measure the Spectrogram of a Supercontinuum Light Pulse. Appl Phys Lett 96, 021102 (2010)

19. Z. Yang, C. Ko, V. Balakrishnan, G. Gopalakrishnan, and S. Ramanathan, Dielectric and Carrier Transport Properties of Vanadium Dioxide Thin Films across the Phase Transition Utilizing Gated Capacitor Devices. Phys Rev B 82, 205101 (2010)

20. I. H. Inoue and M. J. Rozenberg, Taming the Mott Transition for a Novel Mott Transistor. Adv Funct Mater 18, 2289 (2008)

21. D. Ruzmetov, G. Gopalakrishnan, C. H. Ko, V. Narayanamurti, and S. Ramanathan, Three-Terminal Field Effect Devices Utilizing Thin Film Vanadium Oxide as the Channel Layer. J Appl Phys 107, 114516 (2010)

22. J. Son, S. Rajan, S. Stemmer, and S. J. Allen, A Heterojunction ModulationDoped Mott Transistor. J Appl Phys 110, 084503 (2011)

23. M. Nakano, K. Shibuya, D. Okuyama, T. Hatano, S. Ono, M. Kawasaki, Y. Iwasa, and Y. Tokura, Collective Bulk Carrier Delocalization Driven by Electrostatic Surface Charge Accumulation. Nature 487, 459 (2012)

24. S. Luryi, J. Xu, and A. Zaslavsky, Future Trends in Microelectronics: The Nano Millennium. (Wiley, USA, 2002).

25. C. H. Ahn, A. Bhattacharya, M. Di Ventra, J. N. Eckstein, C. D. Frisbie, M. E. Gershenson, A. M. Goldman, I. H. Inoue, J. Mannhart, A. J. Millis, A. F. Morpurgo, D. Natelson, and J. M. Triscone, Electrostatic Modification of Novel Materials. Rev Mod Phys 78, 1185 (2006)

26. B. L. Chamberland and P. S. Danielson, Alkaline-Earth Vanadium (IV) Oxides Having $\mathrm{AVO}_{3}$ Composition. J Solid State Chem 3, 243 (1971)

27. S. Jin, T. H. Tiefel, M. Mccormack, R. A. Fastnacht, R. Ramesh, and L. H. Chen, Thousandfold Change in Resistivity in Magnetoresistive La-Ca-Mn-O Films. Science 264, 413 (1994)

28. J. G. Bednorz and K. A. Muller, Possible High-T $\mathrm{T}_{\mathrm{c}}$ Superconductivity in the BaLa-Cu-O System. Z Phys B Con Mat 64, 189 (1986)

29. V. M. Goldschmidt, Geochemistry. (Oxford University Press, Oxford, 1958).

30. I. H. Inoue, Electrostatic Carrier Doping to Perovskite Transition-Metal Oxides. Semicond Sci Tech 20, S112 (2005)

31. I. H. Inoue, I. Hase, Y. Aiura, A. Fujimori, Y. Haruyama, T. Maruyama, and Y. Nishihara, Systematic Development of the Spectral-Function in the $3 d^{1}$ MottHubbard System $\mathrm{Ca}_{1-x} \mathrm{Sr}_{x} \mathrm{VO}_{3}$. Phys Rev Lett 74, 2539 (1995)

32. I. H. Inoue, O. Goto, H. Makino, N. E. Hussey, and M. Ishikawa, Bandwidth Control in a Perovskite-Type $3 d^{1}$-Correlated Metal $\mathrm{Ca}_{1-x} \mathrm{Sr}_{x} \mathrm{VO}_{3}$. I. Evolution of the Electronic Properties and Effective Mass. Phys Rev B 58, 4372 (1998)

33. P. Zubko, S. Gariglio, M. Gabay, P. Ghosez, and J. M. Triscone, Interface Physics in Complex Oxide Heterostructures. Annu Rev Conden Ma P 2, 141 (2011)

34. J. Biscaras, N. Bergeal, A. Kushwaha, T. Wolf, A. Rastogi, R. C. Budhani, and J. Lesueur, Two-Dimensional Superconductivity at a Mott Insulator/Band Insulator Interface $\mathrm{LaTiO}_{3} / \mathrm{SrTiO}_{3}$. Nat Commun 1, 89 (2010) 
35. H. U. Habermeier, Thin Films of Perovskite-Type Complex Oxides. Mater Today 10, 34 (2007)

36. R. Martin, Electronic Structure: Basic Theory and Practical Methods. (University Press, Cambridge, 2004).

37. C. Kittel, Introduction to Solid State Physics, 8th ed. (Wiley, USA, 2004).

38. J. Zaanen, G. A. Sawatzky, and J. W. Allen, Band-Gaps and Electronic-Structure of Transition-Metal Compounds. Phys Rev Lett 55, 418 (1985)

39. J. Hubbard, Electron Correlations in Narrow Energy Bands. Proc. Phys. Soc. London, Sect. A 276, 238 (1963)

40. A. Fujimori, http://wyvern.phys.s.u-tokyo.ac.jp/f/lecture/srrc/SRRC MIT.pdf.

41. R. E. Thorne, Charge-Density-Wave Conductors. Phys Today 49, 42 (1996)

42. Y. Zhou and S. Ramanathan, Correlated Electron Materials and Field Effect Transistors for Logic: A Review. Crit Rev Solid State 38, 286 (2013)

43. R. E. Peierls, More Surprises in Theoretical Physics. (Princeton University Press, Oxford, 1991).

44. J. Chaussy, P. Haen, J. C. Lasjaunias, P. Monceau, G. Waysand, A. Waintal, A. Meerschaut, P. Molinie, and J. Rouxel, Phase Transitions in $\mathrm{NbSe}_{3}$. Solid State Commun 20, 759 (1976)

45. J. P. Pouget, S. Kagoshima, C. Schlenker, and J. Marcus, Evidence for a Peierls Transition in the Blue Bronzes $\mathrm{K}_{0.30} \mathrm{MoO}_{3}$ and $\mathrm{Rb}_{0.30} \mathrm{MoO}_{3}$. J Phys Lett-Paris 44, L113 (1983)

46. S. van Smaalen, The Peierls Transition in Low-Dimensional Electronic Crystals. Acta Crystallogr A 61, 51 (2005)

47. A. Lagendijk, B. van Tiggelen, and D. S. Wiersma, Fifty Years of Anderson Localization. Phys Today 62, 24 (2009)

48. T. Schwartz, G. Bartal, S. Fishman, and M. Segev, Transport and Anderson Localization in Disordered Two-Dimensional Photonic Lattices. Nature 446, 52 (2007)

49. N. Mott, The Mobility Edge since 1967. J Phys C Solid State 20, 3075 (1987)

50. H. Falcon, J. A. Alonso, M. T. Casais, M. J. Martinez-Lope, and J. SanchezBenitez, Neutron Diffraction Study, Magnetism and Magnetotransport of Stoichiometric $\mathrm{CaVO}_{3}$ Perovskite with Positive Magnetoresistance. J Solid State Chem 177, 3099 (2004)

51. J. Son, Electrical Transport in Nanoscale Complex Oxide Thin Films: $\mathrm{SrTiO}_{3}$ and $\mathrm{RNiO}_{3}$. (PhD Dissertation, University of California, Santa Barbara, 2011).

52. A. Fukushima, F. Iga, I. H. Inoue, K. Murata, and Y. Nishihara, Annealing Effects on Transport and Magnetic Properties of Perovskite-Type Vanadium Oxide $\mathrm{CaVO}_{3}$. J Phys Soc Jpn 63, 409 (1994)

53. J. Garciajaca, J. I. R. Larramendi, M. Insausti, M. I. Arriortua, and T. Rojo, Synthesis, Crystal Structure and Transport Properties of a New Nonstoichiometric $\mathrm{CaVO}_{3+\text { delta }}$ Phase. J Mater Chem 5, 1995 (1995)

54. Y. Ueda, Oxygen Nonstoichiometry, Structures, and Physical Properties of $\mathrm{CaVO}_{3 \text {-delta }}$ I. A Series of New Oxygen-Deficient Phases. J Solid State Chem 135, 36 (1998) 
55. Y. Uyeda, H. Yasuoka, and Y. Ueda, The Effects of the Oxygen Defects in $\mathrm{CaVO}_{3-x}$ System. J Magn Magn Mater 177, 1391 (1998)

56. M. Liberati, R. V. Chopdekar, V. Mehta, E. Arenholz, and Y. Suzuki, Epitaxial Growth and Characterization of $\mathrm{CaVO}_{3}$ Thin Films. J Magn Magn Mater 321, 2852 (2009)

57. P. Dougier, J. C. C. Fan, and J. B. Goodenough, Study of Magnetic, Electrical and Optical Properties of Phases of Perovskite Structure $\mathrm{SrVO}_{2.90}$ and $\mathrm{SrVO}_{3}$. J Solid State Chem 14, 247 (1975)

58. M. Onoda, H. Ohta, and H. Nagasawa, Metallic Properties of Perovskite Oxide $\mathrm{SrVO}_{3}$. Solid State Commun 79, 281 (1991)

59. V. Giannakopoulou, P. Odier, J. M. Bassat, and J. P. Loup, $\mathrm{SrVO}_{3}$ and $\mathrm{Sr}_{2} \mathrm{VO}_{4}$, Electrical Properties Below and Above Room T. Solid State Commun 93, 579 (1995)

60. H. Ishiwara and K. Jyokyu, Formation of Conductive $\mathrm{SrVO}_{3}$ Films on $\mathrm{Si}$ Substrates. Jpn J Appl Phys 30, L2059 (1991)

61. D. H. Kim, D. W. Kim, B. S. Kang, T. W. Noh, D. R. Lee, K. B. Lee, and S. J. Lee, Electrical Properties of $\mathrm{SrVO}_{3} / \mathrm{SrTiO}_{3}$ Superlattices Grown by Laser Molecular Beam Epitaxy. Solid State Commun 114, 473 (2000)

62. D. W. Kim, D. H. Kim, T. W. Noh, K. Char, J. H. Park, K. B. Lee, and H. D. Kim, Interface Chemistry and Electrical Properties of $\mathrm{SrVO}_{3} / \mathrm{LaAlO}_{3}$ Heterostructures. J Appl Phys 88, 7056 (2000)

63. J. A. Moyer, C. Eaton, and R. Engel-Herbert, Highly Conductive $\mathrm{SrVO}_{3}$ as a Bottom Electrode for Functional Perovskite Oxides. Adv Mater 25, 3578 (2013)

64. H. C. Nguyen and J. B. Goodenough, Localized-Itinerant Electronic Transition in the Perovskite System $\mathrm{La}_{1-x} \mathrm{Ca}_{x} \mathrm{VO}_{3}$. Phys Rev B 52, 8776 (1995)

65. K. Maiti, N. Y. Vasanthacharya, and D. D. Sarma, Doping Dependence of Transport and Magnetic Properties in $\mathrm{La}_{1-x} \mathrm{Ca}_{x} \mathrm{VO}_{3}$. J Phys-Condens Mat 9, 7507 (1997)

66. X. Zhang, Y. Zhang, X. M. Wang, X. X. An, C. P. Chen, and X. P. Jing, Transport and Magnetic Properties in the $\mathrm{Gd}_{1-x} \mathrm{Ca}_{x} \mathrm{VO}_{3}$ System. Jpn J Appl Phys 50, $101102(2011)$

67. F. Inaba, T. Arima, T. Ishikawa, T. Katsufuji, and Y. Tokura, Change of Electronic Properties on the Doping-Induced Insulator-Metal Transition in $\mathrm{La}_{1}$ ${ }_{x} \mathrm{Sr}_{x} \mathrm{VO}_{3}$. Phys Rev B 52, R2221 (1995)

68. S. Miyasaka, T. Okuda, and Y. Tokura, Critical Behavior of Metal-Insulator Transition in $\mathrm{La}_{1-x} \mathrm{Sr}_{x} \mathrm{VO}_{3}$. Phys Rev Lett 85, 5388 (2000)

69. T. M. Dao, P. S. Mondal, Y. Takamura, E. Arenholz, and J. Lee, Metal-Insulator Transition in Low Dimensional $\mathrm{La}_{0.75} \mathrm{Sr}_{0.25} \mathrm{VO}_{3}$ Thin Films. Appl Phys Lett 99, $112111(2011)$

70. K. Yoshimatsu, T. Okabe, H. Kumigashira, S. Okamoto, S. Aizaki, A. Fujimori, and M. Oshima, Dimensional-Crossover-Driven Metal-Insulator Transition in $\mathrm{SrVO}_{3}$ Ultrathin Films. Phys Rev Lett 104, 147601 (2010)

71. S. Okamoto, Anomalous Mass Enhancement in Strongly Correlated Quantum Wells. Phys Rev B 84, 201305(R) (2011) 
72. K. Yoshimatsu, K. Horiba, H. Kumigashira, T. Yoshida, A. Fujimori, and M. Oshima, Metallic Quantum Well States in Artificial Structures of Strongly Correlated Oxide. Science 333, 319 (2011)

73. M. Ohring, Materials Science of Thin Films, 2nd ed. (Academic Press, San Diego, 2001).

74. D. P. Norton, Synthesis and Properties of Epitaxial Electronic Oxide Thin-film Materials. Mat Sci Eng R 43, 139 (2004)

75. P. R. Willmott, Deposition of Complex Multielemental Thin Films. Prog Surf Sci 76, 163 (2004)

76. H. M. Christen and G. Eres, Recent Advances in Pulsed-Laser Deposition of Complex Oxides. J Phys-Condens Mat 20, 264005 (2008)

77. M. Nistor and N. B. Mandache, The Channel-Spark Discharge as a Pulsed, Intense Electron and X-Ray Source. Rom Rep Phys 60, 615 (2008)

78. M. Strikovski and K. S. Harshavardhan, Parameters that Control Pulsed Electron Beam Ablation of Materials and Film Deposition Processes. Appl Phys Lett 82, 853 (2003)

79. S. D. Kovaleski, R. M. Gilgenbach, L. K. Ang, and Y. Y. Lau, Electron Beam Ablation of Materials. J Appl Phys 86, 7129 (1999)

80. Neocera Official Website: http://www.neocera.com/Thin Film/ped options.html.

81. M. Nistor, N. B. Mandache, and J. Perriere, Pulsed Electron Beam Deposition of Oxide Thin Films. J Phys D Appl Phys 41, 165205 (2008)

82. M. Strikovski and J. H. Miller, Pulsed Laser Deposition of Oxides: Why the Optimum Rate is about 1 Angstrom Per Pulse. Appl Phys Lett 73, 1733 (1998)

83. M. Druminski, Optimization of the Deposition Conditions for Epitaxial Silicon Films on Czochralski Sapphire in the Silane-Hydrogen System. J Electrochem Soc 127, 957 (1980)

84. Q. D. Jiang, F. C. Matacotta, G. Masciarelli, F. Fuso, E. Arimondo, M. C. Konijnenberg, G. Muller, C. Schultheiss, and G. Sandrin, Characterization and inSitu Fluorescence Diagnostic of the Deposition of $\mathrm{YBa}_{2} \mathrm{Cu}_{3} \mathrm{O}_{7-x}$ Thin Films by Pseudo-Spark Electron-Beam Ablation. Supercond Sci Tech 6, 567 (1993)

85. L. M. Chen, B. Xu, P. Chen, Z. P. Chen, and R. Zhang, Controllable Formation of Particulates and the Effects on Superconductivity of $\mathrm{La}_{1.85} \mathrm{Sr}_{0.15} \mathrm{CuO}_{4}$ Film Grown by the Pulsed Electron Deposition Method. J Supercond Nov Magn 25, 951 (2012)

86. R. J. Choudhary, S. B. Ogale, S. R. Shinde, V. N. Kulkarni, T. Venkatesan, K. S. Harshavardhan, M. Strikovski, and B. Hannoyer, Pulsed-Electron-Beam Deposition of Transparent Conducting $\mathrm{SnO}_{2}$ Films and Study of Their Properties. Appl Phys Lett 84, 1483 (2004)

87. P. H. Quang, N. D. Sang, and D. Q. Ngoc, Pulsed Electron Beam Deposition of Transparent Conducting Al-Doped ZnO Films. Thin Solid Films 520, 6455 (2012)

88. R. Comes, M. Gu, M. Khokhlov, H. X. Liu, J. W. Lu, and S. A. Wolf, Electron Molecular Beam Epitaxy: Layer-by-Layer Growth of Complex Oxides via Pulsed Electron-Beam Deposition. J Appl Phys 113, 023303 (2013) 
89. L. W. Martin, Y. H. Chu, and R. Ramesh, Advances in the Growth and Characterization of Magnetic, Ferroelectric, and Multiferroic Oxide Thin Films. Mat Sci Eng R 68, 89 (2010)

90. D. Kirkwood, Growth and Development of Meta-Multiferroic Systems using Pulsed Electron Deposition (PhD Dissertation, University of Virginia, 2010).

91. R. Comes, Self-Assmebled Epitaxial Multiferroic Oxide Nanostructures Grown by Pulse Electron Deposition (PhD Dissertation, University of Virginia 2013).

92. M. Kawasaki, K. Takahashi, T. Maeda, R. Tsuchiya, M. Shinohara, O. Ishiyama, T. Yonezawa, M. Yoshimoto, and H. Koinuma, Atomic Control of the $\mathrm{SrTiO}_{3}$ Crystal Surface. Science 266, 1540 (1994)

93. K. Rabe, Ch. H. Ahn, and J.-M. Triscone, Physics of Ferroelectrics: A Modern Perspective. (Springer Science \& Business Media, Berlin; New York, 2007).

94. G. S. Rohrer, Structure and Bonding in Crystalline Materials. (Cambridge University Press, Cambridge, UK, 2001).

95. T. L. Alford, L. C. Feldman, and J. W. Mayer, Fundamentals of Nanoscale Film Analysis. (Springer Science \& Business Media, New York, USA, 2007).

96. D. K. Bowen and B. K. Tanner, High Resolution X-Ray Diffractometry And Topography. (CRC Press, UK, 1998).

97. A. Gibaud and S. Hazra, X-Ray Reflectivity and Diffuse Scattering. Curr Sci India 78, 1467 (2000)

98. D. K. Bowen and B. K. Tanner, X-Ray Metrology in Semiconductor Manufacturing. (CRC Press, USA, 2006).

99. D. T. Brower, R. E. Revay, and T. C. Huang, A Study of X-Ray Reflectivity Data Analysis Methods for Thin Film Thickness Determination. Powder Diffraction 11, 114 (1996)

100. J. J. Rehr and R. C. Albers, Theoretical Approaches to X-Ray Absorption Fine Structure. Rev Mod Phys 72, 621 (2000)

101. F. Hippert, Neutron and X-ray Spectroscopy. (Springer, Netherlands, 2006).

102. N. A. Geisse, AFM and Combined Optical Techniques. Mater Today 12, 40 (2009)

103. N. W. Ashcroft and N. D. Mermin, Solid State Physics. (Brooks/Cole, Austrailia, 1976).

104. U. Pietsch, V. Holy, and T. Baumbach, High-Resolution X-Ray Scattering: From Thin Films to Lateral Nanostructures. (Springer Science \& Business Media, USA, 2004).

105. Z. L. Wang and Z. C. Kang, Functional and Smart Materials: Structural Evolution and Structure Analysis (Plenum Press, New York, 1998).

106. S. A. Hayward, S. A. T. Redfern, and E. K. H. Salje, Order Parameter Saturation in $\mathrm{LaAlO}_{3} . J$ Phys-Condens Mat 14, 10131 (2002)

107. M. H. Jung, I. H. Inoue, H. Nakotte, and A. H. Lacerda, High-Field Properties of Single-Crystal $\mathrm{CaVO}_{3}$. Philos Mag B 82, 801 (2002)

108. J. Son, P. Moetakef, J. M. LeBeau, D. Ouellette, L. Balents, S. J. Allen, and S. Stemmer, Low-Dimensional Mott Material: Transport in Ultrathin Epitaxial $\mathrm{LaNiO}_{3}$ Films. Appl Phys Lett 96, 062114 (2010) 
109. E. Sakai, M. Tamamitsu, K. Yoshimatsu, S. Okamoto, K. Horiba, M. Oshima, and H. Kumigashira, Gradual Localization of Ni $3 d$ States in $\mathrm{LaNiO}_{3}$ Ultrathin Films Induced by Dimensional Crossover. Phys Rev B 87, 075132 (2013)

110. J. W. Matthews and A. E. Blakeslee, Defects in Epitaxial Multilayers: I. Misfit Dislocations. J Cryst Growth 27, 118 (1974)

111. O. Entin-Wohlman, Y. Gefen, and Y. Shapira, Variable-Range Hopping Conductivity in Granular Materials. J Phys C Solid State 16, 1161 (1983)

112. A. M. Goldman and S. A. Wolf, Percolation, Localization, and Superconductivity. (Plenum Press, New York and London, 1984).

113. J.M. Ziman, Electrons and Phonons: The Theory of Transport Phenomena in Solids. (Oxford University Press, USA, 2001).

114. G. Bergmann, Weak Localization in Thin Films: A Time-of-Flight Experiment with Conduction Electrons. Phys Rep 107, 1 (1984)

115. B. L. Altshuler, D. Khmel'nitzkii, A. I. Larkin, and P. A. Lee, Magnetoresistance and Hall Effect in a Disordered Two-Dimensional Electron Gas. Phys Rev B 22, $5142(1980)$

116. H. Kamimura, Theoretical Model on the Interplay of Disorder and Electron Correlations. Supplement of the Progress of Theoretical Physics 72, 206 (1982)

117. P. A. Lee and T. V. Ramakrishnan, Disordered Electronic Systems. Rev Mod Phys 57, 337 (1985)

118. A. Kurobe, Theory of Magnetoresistance in the Anderson-Localised Regime of Semiconductors. J Phys C Solid State 19, 2201 (1986)

119. A. Fujimori, http://wyvern.phys.s.u-tokyo.ac.jp/f/lecture/matscig/matsci4a MIT.pdf.

120. H. D. Zhou and J. B. Goodenough, X-Ray Diffraction, Magnetic, and Transport Study of Lattice Instabilities and Metal-Insulator Transition in $\mathrm{CaV}_{1-x} \mathrm{Ti}_{x} \mathrm{O}_{3}(0<=$ $x<=0.4$ ). Phys Rev B 69, 245118 (2004)

121. K. S. Takahashi, D. Matthey, D. Jaccard, and J. M. Triscone, Transport Properties of Reduced $\mathrm{SrTiO}_{3}$ Single Crystal "Thin Films". Ann Phys-Berlin 13, 68 (2004)

122. H. L. Cai, X. S. Wu, and J. Gao, Effect of Oxygen Content on Structural and Transport Properties in $\mathrm{SrTiO}_{3-x}$ Thin Films. Chem Phys Lett 467, 313 (2009)

123. G. Gopalakrishnan, D. Ruzmetov, and S. Ramanathan, On the Triggering Mechanism for the Metal-Insulator Transition in Thin Film $\mathrm{VO}_{2}$ Devices: Electric Field versus Thermal Effects. J Mater Sci 44, 5345 (2009)

124. Z. Yang, S. Hart, C. Ko, A. Yacoby, and S. Ramanathan, Studies on Electric Triggering of the Metal-Insulator Transition in $\mathrm{VO}_{2}$ Thin Films Between $77 \mathrm{~K}$ and 300 K. J Appl Phys 110, 033725 (2011)

125. H. F. Pen, M. Abbate, A. Fuijmori, Y. Tokura, H. Eisaki, S. Uchida, and G. A. Sawatzky, Electronic Structure of $\mathrm{Y}_{1-x} \mathrm{Ca}_{x} \mathrm{VO}_{3}$ Studied by High-Energy Spectroscopies. Phys Rev B 59, 7422 (1999)

126. H. Wadati, D. G. Hawthorn, J. Geck, T. Z. Regier, R. I. R. Blyth, T. Higuchi, Y. Hotta, Y. Hikita, H. Y. Hwang, and G. A. Sawatzky, Interface Reconstruction in V-Oxide Heterostructures Determined by X-Ray Absorption Spectroscopy. Appl Phys Lett 95, 023115 (2009) 
127. F. M. F. de Groot, J. C. Fuggle, B. T. Thole, and G. A. Sawatzky, $L_{2,3}$ X-RayAbsorption Edges of $d^{0}$ Compounds: $\mathrm{K}^{+}, \mathrm{Ca}^{2+}, \mathrm{Sc}^{3+}$, and $\mathrm{Ti}^{4+}$ in $O_{h}$ (Octahedral) Symmetry. Phys Rev B 41, 928 (1990)

128. P. S. Miedema, H. Ikeno, and F. M. F. de Groot, First Principles Multiplet Calculations of the Calcium $L_{2,3} \mathrm{X}$-Ray Absorption Spectra of $\mathrm{CaO}$ and $\mathrm{CaF}_{2} . J$ Phys-Condens Mat 23, 145501 (2011)

129. Z. Sun, J. F. Douglas, A. V. Fedorov, Y. D. Chuang, H. Zheng, J. F. Mitchell, and D. S. Dessau, A Local Metallic State in Globally Insulating $\mathrm{La}_{1.24} \mathrm{Sr}_{1.76} \mathrm{Mn}_{2} \mathrm{O}_{7}$ Well above the Metal-Insulator Transition. Nat Phys 3, 248 (2007)

130. K. Maiti, D. D. Sarma, M. J. Rozenberg, I. H. Inoue, H. Makino, O. Goto, M. Pedio, and R. Cimino, Electronic Structure of $\mathrm{Ca}_{1-x} \mathrm{Sr}_{x} \mathrm{VO}_{3}$ : A Tale of Two Energy Scales. Europhys Lett 55, 246 (2001)

131. J. Laverock, B. Chen, K. E. Smith, R. P. Singh, G. Balakrishnan, M. Gu, J. W. Lu, S. A. Wolf, R. M. Qiao, W. Yang, and J. Adell, Resonant Soft-X-Ray Emission as a Bulk Probe of Correlated Electron Behavior in Metallic $\mathrm{Sr}_{x} \mathrm{Ca}_{1-x} \mathrm{VO}_{3}$. Phys Rev Lett 111, 047402 (2013)

132. I. H. Inoue, I. Hase, Y. Aiura, A. Fujimori, Y. Haruyama, T. Maruyama, and Y. Nishihara, Systematic Development of the Spectral-Function in the $3 d^{1}$ MottHubbard System $\mathrm{Ca}_{1-x} \mathrm{Sr}_{x} \mathrm{VO}_{3}$. Phys Rev Lett 74, 2539 (1995)

133. D. D. Sarma, O. Rader, T. Kachel, A. Chainani, M. Mathew, K. Holldack, W. Gudat, and W. Eberhardt, Contrasting Behavior of Homovalent-Substituted and Hole Doped-Systems: O K-Edge Spectra from $\mathrm{LaNi}_{1-x} \mathrm{M}_{x} \mathrm{O}_{3}(\mathrm{M}=\mathrm{Mn}, \mathrm{Fe}$, and Co) and $\mathrm{La}_{1-x} \mathrm{Sr}_{x} \mathrm{MnO}_{3}$. Phys Rev B 49, 14238 (1994)

134. M. Kobayashi, K. Tanaka, A. Fujimori, S. Ray, and D. D. Sarma, Critical Test for Altshuler-Aronov Theory: Evolution of the Density of States Singularity in Double Perovskite $\mathrm{Sr}_{2} \mathrm{FeMoO}_{6}$ with Controlled Disorder. Phys Rev Lett 98, 246401 (2007)

135. A. R. Denton and N. W. Ashcroft, Vegard's Law. Phys Rev A 43, 3161 (1991)

136. N. F. Mott, Conduction in Non-Crystalline Materials, 2nd ed. (Clarendon Press, Oxford, 1993).

137. H. Tsuiki, K. Kitazawa, and K. Fueki, The Donor Level of $\mathrm{V}^{4+}$ and the MetalNonmetal Transition in $\mathrm{SrTi}_{1-x} \mathrm{~V}_{x} \mathrm{O}_{3}$. Jpn J Appl Phys 22, 590 (1983)

138. K. Y. Hong, S. H. Kim, Y. J. Heo, and Y. U. Kwon, Metal-Insulator Transitions of $\mathrm{SrTi}_{1-x} \mathrm{~V}_{x} \mathrm{O}_{3}$ Solid Solution System. Solid State Commun 123, 305 (2002)

139. A. Ohtomo and H. Y. Hwang, A High-Mobility Electron Gas at the $\mathrm{LaAlO}_{3} / \mathrm{SrTiO}_{3}$ Heterointerface. Nature 427, 423 (2004)

140. N. Reyren, S. Thiel, A. D. Caviglia, L. F. Kourkoutis, G. Hammerl, C. Richter, C. W. Schneider, T. Kopp, A. S. Ruetschi, D. Jaccard, M. Gabay, D. A. Muller, J. M. Triscone, and J. Mannhart, Superconducting Interfaces Between Insulating Oxides. Science 317, 1196 (2007)

141. A. Ohtomo, D. A. Muller, J. L. Grazul, and H. Y. Hwang, Artificial ChargeModulation in Atomic-Scale Perovskite Titanate Superlattices. Nature 419, 378 (2002) 
142. U. Pietsch, V. Holy, and T. Baumbach, High-Resolution X-Ray Scattering. (Springer, Berlin, 2004).

143. J. Son and S. Stemmer, Resistive Switching and Resonant Tunneling in Epitaxial Perovskite Tunnel Barriers. Phys Rev B 80, 035105 (2009)

144. J. Son, J. M. LeBeau, S. J. Allen, and S. Stemmer, Conductivity Enhancement of Ultrathin $\mathrm{LaNiO}_{3}$ Films in Superlattices. Appl Phys Lett 97, 202109 (2010)

145. B. I. Shklovskii and A. L. Efros, Electronic Properties of Doped Semiconductors. (Springer-Verlag, Berlin, 1984).

146. D. H. Kim, D. W. Kim, B. S. Kang, T. W. Noh, D. R. Lee, K. B. Lee, and S. J. Lee, Electrical Properties of $\mathrm{SrVO}_{3} / \mathrm{SrTiO}_{3}$ Superlattices Grown by Laser Molecular Beam Epitaxy. Solid State Commun 114, 473 (2000)

147. M. Izumi, Y. Ogimoto, Y. Okimoto, T. Manako, P. Ahmet, K. Nakajima, T. Chikyow, M. Kawasaki, and Y. Tokura, Insulator-Metal Transition Induced by Interlayer Coupling in $\mathrm{La}_{0.6} \mathrm{Sr}_{0.4} \mathrm{MnO}_{3} / \mathrm{SrTiO}_{3}$ Superlattices. Phys Rev B 64, 064429 (2001)

148. W. C. Sheets, B. Mercey, and W. Prellier, Effect of Charge Modulation in $\left(\mathrm{LaVO}_{3}\right)_{\mathrm{m}}\left(\mathrm{SrVO}_{3}\right)_{\mathrm{n}}$ Superlattices on the Insulator-Metal Transition. Appl Phys Lett 91, 192102 (2007)

149. P. Horsch, A. M. Oles, L. F. Feiner, and G. Khaliullin, Evolution of Spin-OrbitalLattice Coupling in the $\mathrm{RVO}_{3}$ Perovskites. Phys Rev Lett 100, 167205 (2008)

150. W. Choi, T. Sands, and K. Y. Kim, Epitaxial Growth of Semiconducting $\mathrm{LaVO}_{3}$ Thin Films. J Mater Res 15, 1 (2000)

151. A. Masuno, T. Terashima, and M. Takano, Epitaxial Growth of Perovskite-Type $\mathrm{LaVO}_{3}$ Thin Films on Various Substrates by the PLD Method. Solid State Ionics 172, 275 (2004) 